Os números e as funções generalizadas plenas de Colombeau: aspectos algébricos topológicos e analíticos

Antonio Ronaldo Gomes Garcia

TESE APRESENTADA

AO

INSTITUTO DE MATEMÁTICA E ESTATÍSTICA

DA

UNIVERSIDADE DE SÃO PAULO

PARA

OBTENÇÃO DO GRAU DE DOUTOR

EM

CIÊNCIAS

Área de concentração: Matemática pura

Orientador: Prof. Dr. Orlando Stanley Juriaans

Durante a elaboraçâo deste trabalho o autor recebeu auxilio financeiro da CAPES-UERN.

São Paulo, 31 de outubro de 2006 


\title{
Os números e as funções generalizadas plenas de Colombeau: Aspectos algébricos topológicos e analíticos
}

\author{
Este exemplar corresponde à redação \\ final da tese devidamente corrigida \\ e defendida por Antonio Ronaldo Gomes Garcia \\ e aprovada pela comissão julgadora.
}

São Paulo, 31 de outubro de 2006.

Banca examinadora:

- Prof. Dr. Orlando Stanley Juriaans (orientador) - IME - USP

- Prof. Dr. Alfredo Jorge Aragona Vallejo - IME - USP

- Prof. Dr. Francisco Villareal Alvarado - FEIS - UNESP

- Prof. Dr. Mario Carvalho de Matos - IMECC - UNICAMP

- Prof. Dr. José Gomes de Assis - UFPB. 
A minha esposa (Maria do Carmo), mãe (Maria das Dores) e filhos (Raquel, Gelson e Inácio) pelo apoio e confiança durante todos estes anos. 


\section{Agradecimentos}

Inicialmente quero agradecer a Deus, por minha existência e pela coragem que ele me deu para enfrentar mais esta intensa batalha que foi meu doutorado em matemática. $\mathrm{Na}$ verdade nada disso seria possível sem que a fé estivesse presente em cada minuto da minha vida, possibilitando assim transpor os mais difíceis obstáculos que nunca negaram em aparecer para condimentar meu sucesso.

Agradeço ao órgão de fomento, CAPES, pelo suporte financeiro durante os quatro anos que duraram minha estada em São Paulo. E, na oportunidade quero mostrar que sou grato as instituições de ensino superior Universidade do Estado Rio Grande do Norte UERN e Universidade de São Paulo - USP. A primeira por ter me liberado para fazer meu doutoramento e a segunda por ter suas portas abertas para me receber com toda a sua grandiosa estrutura acadêmica e científica. Estrutura esta necessárias ao desenvolvimento desta tese.

Sou grato ao meu Orientador, Prof. Dr. Orlando Stanley Juriaans, pelo tão bem sucedido projeto de pesquisa que culminou neste trabalho de tese que ora apresentamos. Sua amizade, seus conselhos e sua paciência foram fundamentais para o meu amadurecimento científico (em matemática) o que resultou na vitória de mais esta etapa da minha formação acadêmica.

Ao Prof. Dr. Alfredo Jorge Aragona Vallejo que contribui com muito dos seus manuscritos para a realização desta tese; sou grato. Sou grato, também, pela sua amizade, seus conselhos e a força que ele me deu para uma boa realização desta obra. Este professor juntamente com o Prof. Dr. Oswaldo Rio Branco de Oliveira, a quem tenho muito a 
agradecer, teve também um papel importante na minha formação de mestre em ciências (área: matemática).

Aos professores do Instituto de Matemática e Estatística - IME - USP que de uma forma, ou de outra contribuíram para o meu amadurecimento em matemática o que, também, culminou na realização desta tese. Até os pensamentos de alguns de forma negativa ao meu nome, foram essenciais para me motiva a mostrar o contrário do que eles pensavam. Deixo aqui um agrdecimento especial a Prof. Dra. Roselir Fernandez como uma das integrantes do grupo de funções generalizadas, e suplente de minha banca julgadora de tese. Na oportunidade, agradeço ao Prof. Dr. Ernandes Rocha de Oliveira membro suplente na minha banca examinadora, infelizmente não pode participar mas se mostrou pronto.

Aos professores da banca examinadora, ainda não citados, a saber, Prof. Dr. Francisco Villareal Alvarado, Prof. Dr. Mario Carvalho de Matos e Prof. Dr. José Gomes de Assis que não mediram esforços em participar e contribuir com suas esperiências matemáticas em minha tese.

Aos funcionários do IME - USP por suas valiosas prestações de serviços em todas as áreas. Principalmente, as meninas da sala do café, a saber: Jovita, Dona Maria e Dalvina.

Aos meus colegas alunos do IME - USP. Vou lembrar alguns nomes e aqueles que não lembrei tentem me desculpar e se vejam entre aqueles que lembrei: Samuel Gomes, Jocirei Dias, Olga, Rudimar, Bárbara, José Vilhena, Raul, Panzarelli, Danielle, Sávio, Odirlei, Andrea, Márcio, David, Emivan, Walter, Herivelto, Calixto, Edson, Samir (os quatro últimos são orientandos do Orlando), Carlos Eider, Carlão, Severino Horácio, Elmo, Iran, Robson Martins (os três últimos são Goianos), Gladys, Joselito, Marlon e Gerard (peruano).

A minha esposa Maria do Carmo Pereira de Souza e filhos, a saber: Hozana Raquel de Medeiros Garcia, Gelson Iezzi de Medeiros Garcia e Inácio Antônio de Souza Garcia. Na oportunidade minha mãe Maria das Dores Gomes Garcia e irmãos, a saber: Maria Lúcia Gomes Garcia, Marta Rosa Gomes Garcia, Francisco de Assis Gomes Garcia e José Olímpio Gomes Garcia. Todos pela confiança e paciência prestada durante toda a realização deste trabalho. Ao meu pai Espedito Alves Garcia (in memorian).

Aos meus colegas externos ao IME - USP que também foram fundamentais para o 
desenvolvimento da tese. Citamos alguns: Edna, Adriana, Jefferson, os professores da FANAT-UERN (em especial Josildo, Jeovanizélio, Braz e Elias), os professores do Departamento de Matemática e Estatística da UFMT, os professores do Departamento de Ciências Ambientais da UFERSA. Nesta última devo ainda destacar meus agradecimentos ao professor Walter Martins Rodrigues por sua atenção nos seminários desenvolvidos em Mossoró-RN e suas valiosas discussões sobre este trabalho. 


\section{Resumo}

Denotamos por $\overline{\mathbb{K}}_{f}$ o anel comutativo com unidade dos números generalizados pleno de Colombeau. ( $\mathbb{K}$ denotará $\mathbb{R}$ ou $\mathbb{C}$.) Este anel pode ser munido com uma ultra-métrica de tal modo que $\overline{\mathbb{K}}_{f}$ é um anel topológico. Denotamos por $\mathcal{G}_{f}(\Omega)$ a álgebra das funções generalizadas plena de Colombeau, $\Omega$ é um subconjunto aberto não vazio de $\mathbb{R}^{n}$, e denotamos por $\mathcal{G}_{f_{c}}(\Omega)$ aquelas que tem suporte compacto. Mostramos que $\mathcal{G}_{f_{c}}(\Omega)$ é denso em $\mathcal{G}_{f}(\Omega)$ com a topologia cortante que definimos para esta álgebra. Quando $\emptyset \neq \Omega \subset \mathbb{R}^{n}$ é aberto e limitado denotamos por $\mathcal{G}_{f}(\bar{\Omega})$ a álgebra das funções generalizadas plena de Colombeau sobre o fecho topológico de $\Omega$. Mostramos que $\mathcal{G}_{f}(\bar{\Omega})$ munido com sua topologia é completo. (Ver [4].) Ainda neste trabalho, generalizamos um resultado de existência e unicidade para um problema de valor inicial e de fronteira do tipo parabólico devido a Colombeau e Langlais [10]. Para isto aplicamos os resultados topológicos de densidade e completude mencionados acima. 


\section{Abstract}

Let $\overline{\mathbb{K}}_{f}$ denote the commutative ring with unity of Colombeau's full generalized numbers. (Here $\mathbb{K}$ will denote either $\mathbb{R}$ or $\mathbb{C}$.) This ring can be endowed with an ultra-metric in such a way that $\overline{\mathbb{K}}_{f}$ is a topological ring. Let $\mathcal{G}_{f}(\Omega)$ denote the algebra of Colombeau's full generalized functions, $\Omega$ is a non-void open subset of $\mathbb{R}^{n}$, and let $\mathcal{G}_{f_{c}}(\Omega)$ denote that have compact support. We show that $\mathcal{G}_{f_{c}}(\Omega)$ is dense in $\mathcal{G}_{f}(\Omega)$ with the sharp topologies that we define for this algebra. When $\emptyset \neq \Omega \subset \mathbb{R}^{n}$ is opened and limited we denote for $\mathcal{G}_{f}(\bar{\Omega})$ the algebra of Colombeau's full generalized functions on the topological lath of $\Omega$. We show that $\mathcal{G}_{f}(\bar{\Omega})$ endowed with its topology is complete. (See [4].) Still in this work, we generalize a result of existence and unicity for a problem of initial value and boundary of the parabolic type which had Colombeau and Langlais [10]. For this we above apply the topological results of mentioned density and completude. 


\section{Conteúdo}

$\begin{array}{ll}\text { Introdução } & 1\end{array}$

Notações $\quad \therefore \quad 5$

1 Topologia e álgebra dos números generalizados plenos de Colombeau 9

1.1 A topologia cortante escalar plena . . . . . . . . . . . . . . . . 10

1.2 Propriedades algébricas de $\overline{\mathbb{K}}_{f} \ldots \ldots \ldots \ldots \ldots \ldots$. . . . . . . . . . . . 21

1.3 Tipos especiais de funções características . . . . . . . . . . . . . . . . 28

2 Relação de ordem sobre $\overline{\mathbb{R}}_{f}$ e outras propriedades algébricas de $\overline{\mathbb{K}}_{f} \quad 47$

2.1 Relação de ordem sobre $\overline{\mathbb{R}}_{f} \ldots \ldots \ldots \ldots \ldots$. . . . . . . . . . 47

2.2 Outras propriedades algébricas de $\overline{\mathbb{K}}_{f} \ldots \ldots \ldots \ldots$

3 A topologia cortante sobre a álgebra das funções generalizadas plenas $\begin{array}{ll}\text { de Colombeau } & 57\end{array}$

3.1 A álgebra das funções generalizadas . . . . . . . . . . . . . . . . . . 58

3.1.1 Funções generalizadas sobre um conjunto aberto . . . . . . . . . . 58

3.1.2 Funções generalizadas sobre a fronteira de um conjunto aberto . . . 60

3.2 A topologia cortante sobre $\mathcal{G}_{f}(\Omega) \ldots \ldots \ldots \ldots$ 
3.3 Resultados sobre convergência em $\left(\mathcal{G}_{f}(\Omega), \tau_{\Omega f}\right) \ldots \ldots \ldots \ldots \ldots$

3.4 A topologia cortante sobre $\mathcal{G}_{f}(\bar{\Omega}), \Omega \subset \mathbb{R}^{n}$ aberto limitado . . . . . . 78

4 Solução generalizada para um problema de valor inicial e de fronteira $\begin{array}{lr}\text { do tipo parabólico } & 89\end{array}$

4.1 Desenvolvimento histórico do problema . . . . . . . . . . . . . . . . 89

4.2 A solução generalizada dada por Colombeau e Langlais . . . . . . . . . . . 92

4.3 Uma aplicação das topologias cortantes $\left(\mathcal{G}_{f}(\Omega), \tau_{\Omega f}\right)$ e $\left(\mathcal{G}_{f}(\bar{Q}), \tau_{\bar{Q} b}\right) \ldots .94$

Referências Bibliográficas $\quad 99$

Índice Remissivo $\quad 101$ 


\section{Introdução}

Neste trabalho nos propomos, inicialmente, a pesquisar os aspectos topológicos e algébricos do anel dos números generalizados plenos de Colombeau $\overline{\mathbb{K}}_{f}$. O nosso objetivo é, portanto, transferir os resultados obtidos por Aragona e Juriaans [5], onde se pesquisou algumas propriedades estruturais do anel dos números generalizados simplificados de Colombeau $\overline{\mathbb{K}}$, para $\overline{\mathbb{K}}_{f}$. A motivação para os estudos algébricos surgiu quando conseguimos dar uma definição para o conjunto $\mathcal{S}_{f}$ (ver Definição 1.3.1) que faz o "papel" do conjunto $\mathcal{S}$ definido por Aragona e Juriaans [5]. E a motivação para os estudos topológicos vieram de estudos recentes de D. Scarpalézos (ver $[15,16,14]$ ) que introduziu topologias metrizáveis sobre $\overline{\mathbb{K}}$ e sobre $\mathcal{G}(\Omega)$ (chamadas de "topologias cortantes") para a qual todas as operações envolvidas na estrutura da $\overline{\mathbb{K}}$-álgebra $\mathcal{G}(\Omega)$ são contínuas e, também do conceito de associação entre elementos de $\overline{\mathbb{K}}_{f}$ que podemos ver, por exemplo, no trabalho do Kunzinger [12].

Num segundo momento; baseado nos trabalhos de Aragona, Juriaans, Oliveira e Scarpalézos [6] e Aragona, Fernandez e Juriaans [3], estudamos uma relação de ordem sobre o anel dos números generalizados plenos de Colombeau, quando o corpo de escalares é real, onde obtemos alguns resultados importantes na teoria os quais deixaremos mais claro no decorre desta pesquisa.

Num terceiro momento, visando generalizar os resultados de existência e unicidade de solução generalizada para um problema de valor inicial e de fronteira, abreviadamente PVIF, do tipo parabólico obtido por Colombeau e Langlais [10], estudamos a topologia cortante da álgebra das funções generalizadas plenas de Colombeau, quando definida sobre o aberto, não-vazio, $\Omega \subset \mathbb{R}^{n}, \mathcal{G}_{f}(\Omega)$ e, quando definida sobre o fecho topológico do aberto, 
não-vazio e limitado $\Omega \subset \mathbb{R}^{n}, \mathcal{G}_{f}(\bar{\Omega})$ obtendo, assim alguns resultados sobre convergência nestas álgebras. É importante destacarmos o trabalho de Silva [17], onde reuniu os principais resultados sobre a álgebra das funções generalizadas simplificadas de Colombeau $\mathcal{G}(\Omega)$ e, foi fundamental para nos espelharmos em nossos estudos, principalmente no que diz respeito a construção da topologia cortante sobre $\mathcal{G}_{f}(\Omega)$ que, aparentemente, é diferente daquela apresentada por Aragona, Fernandez e Juriaans [3]. Nosso objetivo maior, consiste em mostrar que com a topologia cortante a álgebra das funções generalizadas plenas de Colombeau sobre o fecho topológico de $\Omega \subset \mathbb{R}^{n}, \mathcal{G}_{f}(\bar{\Omega})$, é completo (ver [4]) e que a álgebra das funções generalizadas plenas de Colombeau sobre $\Omega \subset \mathbb{R}^{n}$ com suporte compacto, $\mathcal{G}_{f_{c}}(\Omega)$, é denso em $\mathcal{G}_{f}(\Omega)$.

Finalmente, aplicamos os fatos topológicos de completude e densidade, já mencionados, para generalizar o trabalho de Colombeau e Langlais [10]. Este trabalho nos mostra uma real importância das funções generalizadas na solução de equações diferenciais parciais não-lineares, uma vez que resolveu um problema desta natureza que não admitia solução fraca (solução no sentido de distribuições) apresentado no artigo de Brezis e Friedman [8].

Para que tenhamos em mão o rumo da nossa pesquisa damos uma breve descrição do que vamos desenvolver ao longo destas linhas.

No Capítulo 1 trabalhamos os aspectos topológicos e algébricos do anel dos números generalizados plenos de Colombeau $\overline{\mathbb{K}}_{f}$. Na Seção 1.1 definimos o anel dos números generalizados plenos de Colombeau $\overline{\mathbb{K}}_{f}$ e definimos, também, uma relação sobre os seus elementos que chamaremos de associação (denotada por $\approx$ ). Esta relação é uma relação de equivalência e é também conhecida como igualdade no sentido fraco entre dois elementos de $\overline{\mathbb{K}}_{f}$ (ver por exemplo [12]). Ainda nesta seção construímos uma noção topológica para $\overline{\mathbb{K}}_{f}$, fazendo uso do conceito de associação (ver [3]). Na Seção 1.2 damos início aos estudos algébricos de $\overline{\mathbb{K}}_{f}$. Como havíamos dito antes estes estudos só foram possíveis graças a definição do conjunto $\mathcal{S}_{f}$ que faz o "papel" do conjunto $\mathcal{S}$ definido por Aragona e Juriaans [5]. Como resultados importantes nesta seção destacamos a Proposição 1.2.2 e seu Corolário 1.2.3 que afirma que o conjunto das unidades de $\overline{\mathbb{K}}_{f}$ é aberto, bem como o Teorema 1.2.9 onde diz que o nil-radical de $\overline{\mathbb{K}}_{f}$ é nulo e, portanto, $\overline{\mathbb{K}}_{f}$ está contido num produto de domínios integrais. Na Seção 1.3, estudamos o conjunto das unidades e os ideais primos e maximais de $\overline{\mathbb{K}}_{f}$; fazendo uma análise cuidadosa do conjunto de zeros dos representantes dos elementos de $\overline{\mathbb{K}}_{f}$, definindo um tipo especial de funções características 
e mostrando que elas estão relacionadas com os ideais primos e maximais de $\overline{\mathbb{K}}_{f}$. Entre os vários resultados obtidos nesta seção destacamos: O radical de Jacbson de $\overline{\mathbb{K}}_{f}$ é nulo (ver Teorema 1.3.20), o conjunto (aberto) das unidades é um subconjunto denso de $\overline{\mathbb{K}}_{f}$ (ver Teorema 1.3.22). Também damos algumas caracterizações para os invertíveis, bem como uma completa descrição dos ideais maximais de $\overline{\mathbb{K}}_{f}$ (Teorema 1.3.21). Este resultado e o da densidade é uma conseqüência do Teorema da aproximação (Teorema 1.3.17).

No Capítulo 2 introduzimos uma relação de ordem parcial sobre $\overline{\mathbb{R}}_{f}$, o anel dos números generalizados plenos de Colombeau sobre $\mathbb{R}$, que mostraremos induzir uma relação de ordem total em todo o corpo de classes residuais. Na Seção 2.1, destacamos os seguintes resultados: A convexidade de ideais (Proposição 2.1.10) e o Teorema 2.1.17, que consideramos o principal resultado desta seção. Na Seção 2.2 damos continuidade aos fatos algébricos estudados no Capítulo 1 e completamos os estudos dos ideais de $\overline{\mathbb{K}}_{f}$ dando uma completa descrição dos ideais primos. Um outro fato que merece destaque é a completa descrição dos idempotentes de $\overline{\mathbb{K}}_{f}$ (ver Teorema 2.2.1) e que este anel não é Von-Neumann regular (ver Teorema 2.2.5).

No Capítulo 3 construímos uma topologia sobre a álgebra das funções generalizadas plenas de Colombeau $\mathcal{G}_{f}(\Omega)$, veremos que esta topologia é Hausdorff e demonstramos alguns resultados sobre convergência em $\left(\mathcal{G}_{f}(\Omega), \tau_{\Omega f}\right)$. Ainda neste capítulo definimos uma topologia sobre $\mathcal{G}_{f}(\bar{\Omega})$ e mostramos que com esta topologia $\mathcal{G}_{f}(\bar{\Omega}), \Omega \subset \mathbb{R}^{n}$ é um aberto não-vazio limitado, é completo (ver [4]). É bom salientar que a álgebra das funções generalizadas plenas de Colombeau sobre $\bar{\Omega}$ (o fecho topológico de $\Omega \subset \mathbb{R}^{n}$ ) está definida para todo aberto $\Omega \subset \mathbb{R}^{n}$ (ver, por exemplo, [10]), mas a topologia não, a topologia exige que $\Omega \subset \mathbb{R}^{n}$ seja um aberto não-vazio limitado. Esta exigência foi fundamental para que tivéssemos uma topologia Hausdorff sobre $\mathcal{G}_{f}(\bar{\Omega})$. Na Seção 3.1 definimos a exaustão de um aberto $\Omega \subset \mathbb{R}^{n}$ e as álgebras das funções generalizadas plenas de Colombeau $\mathcal{G}_{f}(\Omega)$ e $\mathcal{G}_{f}(\bar{\Omega})$. É bom também notar a Proposição 3.1.1, muito conhecida ver, por exemplo, Aragona, Fernandez e Juriaans [3], que caracteriza os elementos moderados e nulos da álgebra via o conceito de exaustão. Na Seção 3.2 chamamos a atenção para a definição do conjunto $\mathrm{A}_{m p}(u)$ e a definição de "valuação" apresentados na Definição 3.2.1, pois é a partir deles e do conceito de associação que definimos a topologia cortante sobre $\mathcal{G}_{f}(\Omega)$. É também importante as Proposições 3.2 .4 e 3.2 .5 que definem a aplicação $D_{m p}$ que mostraremos definir uma pseudo-ultra-métrica. Na Secção 3.3 demonstramos alguns 
resultados sobre convergência em $\left(\mathcal{G}_{f}(\Omega), \tau_{\Omega f}\right)$. Dentre eles destacamos que a álgebra funções generalizadas plenas de Colombeau de suporte compacto, $\mathcal{G}_{f_{c}}(\Omega)$, é denso em $\mathcal{G}_{f}(\Omega)$ (ver Corolário 3.3.9). Finalmente, na Seção 3.4, com a topologia definida sobre $\mathcal{G}_{f}(\bar{\Omega})$, onde $\bar{\Omega}$ é o fecho topológico de $\Omega \subset \mathbb{R}^{n}$, um subconjunto aberto, não-vazio e limitado temos que a álgebra das funções generalizadas plenas de Colombeau sobre $\bar{\Omega}$ é completa (ver Teorema 3.4.13) cuja prova encontramos em [4].

No Capítulo 4, estudamos um problema de valor inicial e de fronteira (PVIF) do tipo parabólico devido a Colombeau e Langlais [10]. Em seu artigo, eles mostraram que se o dado inicial $u_{0}$ é uma função generalizada de suporte compacto, i.e., $u_{0} \in \mathcal{G}_{f_{c}}(\Omega)$ então existe uma única $u \in \mathcal{G}_{f}(\bar{Q})$ solução do PVIF:

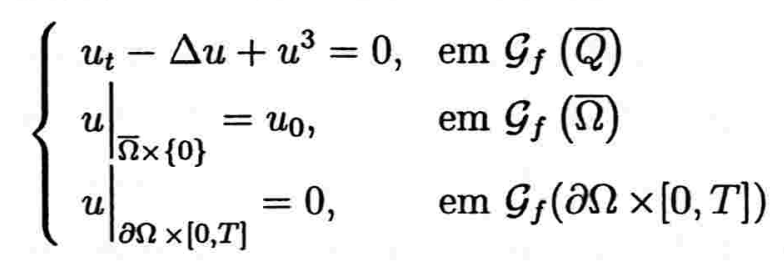

(ver Teoremas 4.2.2 e 4.2.4, Seção 4.2). Nosso objetivo é, portanto, mostrar que o mesmo permanece valendo mesmo que o dado inicial $u_{0}$ não seja, necessariamente, uma função generalizada de suporte compacto, i.e., basta que $u_{0} \in \mathcal{G}_{f}(\Omega)$ (ver Teorema 4.3.1, Seção 4.3). Para isto fazemos uso dos fatos topológicos de densidade e completude obtidos no Capítulo 3. É bom observarmos que tanto no trabalho de Colombeau e Langlais [10] quanto na nossa generalização, estamos considerando as funções generalizadas plenas de Colombeau a valores em $\mathbb{R}$. Como havíamos dito antes este problema é um exemplo que mostra a importância das funções generalizadas na solução de equações diferenciais parciais não lineares, uma vez que Brezis e Friedman mostraram que o problema acima não admite solução fraca (solução no sentido das distribuições) (ver [8]). Na Seção 4.1, damos um desenvolvimento histórico do problema mostrando o que afirmou, em 1983, Brezis e Friedman, [8], com respeito ao PVIF (1), e o avanço dado, em 1990, por Colombeau e Langlais quando mostraram que (1) admite uma única solução generalizada, se o dado inicial é uma função generalizada de suporte compacto (ver [10]). Na Seção 4.2, apresentamos os principais resultados, sem prova, obtidos por Colombeau e Langlais em [10]. Pois, estes resultados serão fundamentais para o nosso propósito. Na Seção 4.3, aplicamos a topologia cortante sobre $\mathcal{G}_{f}(\Omega)$ e $\mathcal{G}_{f}(\bar{\Omega})$ para generalizarmos o trabalho de Colombeau e Langlais [10]. Os principais fatos topológicos aplicados aqui foram a desidade de $\mathcal{G}_{f_{c}}(\Omega)$ em $\mathcal{G}_{f}(\Omega)$ e a completude de $\mathcal{G}_{f}(\bar{\Omega})$ (ver [4]). 


\section{Notações}

Vamos enumerar algumas notações que vamos usar livremente no decorre deste trabalho.

1. $I:=] 0,1], \bar{I}:=[0,1]$ e $\left.I_{\eta}:=\right] 0, \eta[, \forall \eta \in I$.

2. $A \backslash B:=\{a \in A \mid a \notin B\}$.

3. $\mathbb{Q}$, denotará o corpo dos números racionais.

4. $\mathbb{K}$, como é usual, denotará o corpo real (ou complexo), i.e., $\mathbb{R}$ (ou $\mathbb{C}$ ).

5. $\mathbb{N}, \mathbb{Z}$, como é usual, denotará, respectivamente, o conjunto dos números naturais e o conjunto dos números inteiros. $\mathbb{N}^{*}:=\mathbb{N} \backslash\{0\}$ e $\mathbb{Z}^{*}:=\mathbb{Z} \backslash\{0\}$.

6. $\mathbb{K}^{*}:=\mathbb{K} \backslash\{0\}$.

7. $\mathbb{R}_{+}:=\{x \in \mathbb{R} \mid x \geq 0\}$ e $\mathbb{R}_{+}^{*}:=\{x \in \mathbb{R} \mid x>0\}$.

8. $\Omega$ é um subconjunto aberto, não vazio, de $\mathbb{R}^{n}$ e $K \subset \subset \Omega$ significa que $K$ é um subconjunto compacto de $\Omega$. Dada uma função $f: X \rightarrow \mathbb{K}$ e $\emptyset \neq Y \subset X$ definimos $\|f\|_{Y}:=\sup _{x \in K}|f(x)|$, onde $K \subset \subset Y$.

9. $\mathbb{N}^{n}:=\underbrace{\mathbb{N} \times \mathbb{N} \times \cdots \times \mathbb{N}}_{n \text {-vezes }}$. Um elemento $\alpha \in \mathbb{N}^{n}$ é chamado multi-índice.

10. Temos

$$
|\alpha|=\sum_{i=1}^{n} \alpha_{i}
$$


é o comprimento $\alpha \in \mathbb{N}^{n}$ e

$$
\partial^{\alpha}:=\frac{\partial^{|\alpha|}}{\partial_{x_{1}}^{\alpha_{1}} \partial_{x_{2}}^{\alpha_{2}} \ldots \partial_{x_{n}}^{\alpha_{n}}} .
$$

é o operador derivada $\alpha$-ésima, $\alpha=\left(\alpha_{1}, \alpha_{2}, \ldots, \alpha_{n}\right) \in \mathbb{N}^{n}$.

11. Fórmula de Leibniz:

$$
\partial^{\alpha}(u . v)=\sum_{0 \leq \beta \leq \alpha}\left(\begin{array}{l}
\alpha \\
\beta
\end{array}\right) \partial^{\alpha-\beta} u \partial^{\beta} v
$$

onde $\alpha, \beta \in \mathbb{N}^{n}, \beta=\left(\beta_{1}, \beta_{2}, \ldots, \beta_{n}\right)$ e $\alpha=\left(\alpha_{1}, \alpha_{2}, \ldots, \alpha_{n}\right)$ são tais que

$$
\beta \leq \alpha \Leftrightarrow \beta_{i} \leq \alpha_{i}, \forall i=1,2, \ldots, n
$$

12. $\mathbb{R}^{n}:=\underbrace{\mathbb{R} \times \mathbb{R} \times \cdots \times \mathbb{R}}_{n \text {-vezes }}$ é o espaço euclidiano real $n$-dimensional.

13. $\overline{\mathbb{K}}$, denotará o anel dos números generalizados simplificados de Colombeau.

14. $\overline{\mathbb{R}}$, denotará o anel dos números generalizados simplificados de Colombeau sobre o corpo dos números reais.

15. $\overline{\mathbb{C}}$, denotará o anel dos números generalizados simplificados de Colombeau sobre o corpo dos números complexos.

16. $\overline{\mathbb{K}}_{f}$, denotará o anel dos números generalizados plenos de Colombeau.

17. $\overline{\mathbb{R}}_{f}$, denotará o anel dos números generalizados plenos de Colombeau sobre o corpo dos números reais.

18. $\overline{\mathbb{C}}_{f}$, denotará o anel dos números generalizados plenos de Colombeau sobre o corpo dos números complexos.

19. $\mathcal{A}_{0}(\mathbb{R}):=\left\{\varphi \in \mathcal{D}(\mathbb{R}) \mid \int_{0}^{\infty} \varphi(x) \mathrm{d} x=\frac{1}{2}, \varphi\right.$ é par e $\varphi \equiv$ const. em $\left.V_{0}\right\}$, onde $V_{0}$ é uma vizinhança da origem.

20. $\mathcal{A}_{q}(\mathbb{R}):=\left\{\varphi \in \mathcal{A}_{0}(\mathbb{R}) \mid \int_{0}^{\infty} x^{\frac{j}{m}} \varphi(x) \mathrm{d} x=0\right.$, para $\left.1 \leq j, m \leq q\right\}$, onde $q \in \mathbb{N}$.

21. $\mathbb{K}^{*}:=\mathbb{K} \backslash\{0\}$. 
22. Temos que

$$
\Gamma:=\left\{\gamma: \mathbb{N} \rightarrow \mathbb{R}_{+} \mid \gamma(n)<\gamma(n+1), \forall n \in \mathbb{N} \text { e } \lim _{n \rightarrow \infty} \gamma(n)=\infty\right\}
$$

é o conjunto das seqüências estritamente crescentes que divergem quando $n \rightarrow \infty$.

23. $\mathcal{G}(\Omega)$, denotará a álgebra das funções generalizadas simplificadas de Colombeau.

24. $\mathcal{G}_{C}(\Omega)$ é a álgebra das funções generalizadas simplificadas com suporte compacto.

25. $\mathcal{G}_{f}(\Omega)$, denotará a álgebra das funções generalizadas plenas de Colombeau.

26. $\mathcal{G}_{f_{c}}(\Omega)$, denotará a álgebra das funções generalizadas plenas de Colombeau com suporte compacto.

27. $\bar{\Omega}$ é o fecho topológico do aberto $\Omega \subset \mathbb{R}^{n}$. Então $\mathcal{G}(\bar{\Omega})$ é a álgebra das funções generalizadas simplificadas de Colombeau sobre o fecho topológico de $\Omega \subset \mathbb{R}^{n}$ e $\mathcal{G}_{f}(\bar{\Omega})$ é a álgebra das funções generalizadas plenas de Colombeau sobre o fecho topológico de $\Omega \subset \mathbb{R}^{n}$.

28. $\mathcal{D}(\Omega)$ denotará o espaço das funções $\mathrm{C}^{\infty}(\Omega)$ com suporte compacto em $\Omega \subset \mathbb{R}^{n}$.

29. $\mathcal{D}^{\prime}(\Omega)$ denotará o espaço das distribuições. 
Capítulo 1

\section{Topologia e álgebra dos números generalizados plenos de Colombeau}

Neste capítulo objetivamos generalizar os resultados algébricos e topológicos obtidos por Aragona e Juriaans [5] para o anel dos números generalizados plenos de Colombeau $\overline{\mathbb{K}}_{f}$. Na Seção 1.1, vamos construir uma topologia intrínseca para $\overline{\mathbb{K}}_{f}$ utilizando o conceito de associação e, a partir disso, daremos algumas propriedades topológicas deste anel. Esta construção é devido a Aragona, Fenandez e Juriaans [3]. Na Seção 1.2 apresentaremos algumas propriedades algébricas, dentre elas destacamos: $\mathrm{O}$ conjunto das unidades de $\overline{\mathbb{K}}_{f}$ é aberto (ver Teorema 1.2.3), todo ideal maximal de $\overline{\mathbb{K}}_{f}$ é fechado e assim é, também um conjunto raro (ver Teorema 1.2.4), o seu nil-radical é nulo e, portanto, $\overline{\mathbb{K}}_{f}$ está contido num produto de domínios integrais (ver Teorema 1.2.9). Na Seção 1.3 estudamos tipos especiais de funções características mostrando que elas estão relacionadas com os ideais primos e maximais de $\overline{\mathbb{K}}_{f}$. Dentre os resultados obtidos, destacamos: Algumas caracterizações para os invertíveis (ou conjunto das unidades) de $\overline{\mathbb{K}}_{f}$, que denotaremos por $\operatorname{Inv}\left(\overline{\mathbb{K}}_{f}\right)$, o Teorema da aproximação (Teorema 1.3.17) e suas aplicações, a saber: Uma descrição completa dos ideais maximais de $\overline{\mathbb{K}}_{f}$ (Teorema 1.3.21) e mostramos que o conjunto $\operatorname{Inv}\left(\overline{\mathbb{K}}_{f}\right)$ (aberto) é também denso em $\overline{\mathbb{K}}_{f}$ (Teorema 1.3.22). 


\subsection{A topologia cortante escalar plena}

Iniciamos esta seção definindo o anel dos números generalizados plenos de Colombeau $\overline{\mathbb{K}}_{f}$. Definimos uma relação sobre $\overline{\mathbb{K}}_{f}$ chamada de associação $(\approx)$, que é uma relação de equivalência. Esta relação é também conhecida como igualdade no sentido fraco entre dois elementos de $\overline{\mathbb{K}}_{f}$. É a partir desta relação de associação que vamos construir uma noção topológica intrínseca para o anel dos números generalizados plenos de Colombeau. (Ver [3] para mais detalhes.)

As seguintes definições e resultados podemos ver, por exemplo, em [2] (pg. 77-78).

Definição 1.1.1. Definimos

$$
\mathcal{A}_{0}(\mathbb{R} ; \mathbb{K})=\left\{\varphi_{1} \in \mathcal{D}(\mathbb{R}, \mathbb{K}) \mid \varphi_{1} \text { é par, } \varphi_{1} \equiv \text { const. em } V_{0} e \int_{0}^{+\infty} \varphi_{1}(x) \mathrm{d} x=\frac{1}{2}\right\}
$$

onde $V_{0}$ denota uma vizinhança da origem. $E$, se $q \in \mathbb{N}^{*}$, definimos

$$
\mathcal{A}_{q}(\mathbb{R} ; \mathbb{K})=\left\{\varphi_{1} \in \mathcal{A}_{0}(\mathbb{R} ; \mathbb{K}) \mid \int_{0}^{+\infty} x^{\frac{j}{m}} \varphi_{1}(x) \mathrm{d} x=0, \text { para } 1 \leq j, m \leq q\right\}
$$

Quando $\mathbb{K}=\mathbb{R}$, temos a seguinte:

Proposição 1.1.2. O conjunto $\mathcal{A}_{q}(\mathbb{R} ; \mathbb{R})=\mathcal{A}_{q}(\mathbb{R})$ é não-vazio para cada $q \in \mathbb{N}^{*}$. Mais precisamente, para todo $a, b \in \mathbb{R}_{+}$e para cada $q \in \mathbb{N}^{*}$, existe $\varphi_{1} \in \mathcal{A}_{q}(\mathbb{R})$ tal que $\varphi_{1}(a)=b$.

Prova. Ver [2] (pg. 77-78).

A partir do conjunto $\mathcal{A}_{q}(\mathbb{R})$ construímos (ver $[2,12]$ ) classes de funções teste para quaisquer espaços normados, suaves de dimensão finita. Um espaço normado, suave $E$ é entendido como um espaço vetorial de dimensão finita munido de uma norma que é diferenciável sobre $E \backslash\{0\}$. Para $n \in \mathbb{N}$, denotamos por $\omega_{n}$ a área da esfera $(n-1)$ dimensional $S^{n-1}$ em $\mathbb{R}^{n}$ e definimos

$$
c_{n}:= \begin{cases}\frac{2 n}{\omega_{n}} & \text { para } n>1 \\ 1 & \text { para } \mathrm{n}=1\end{cases}
$$

Se $E$ é um espaço normado, suave $n$-dimensional (que enfatizamos escrevendo $E=E_{n}$ ) com norma $\|\cdot\|_{E_{n}}$ então para cada $\varphi_{1} \in \mathcal{A}_{0}(\mathbb{R})$ a aplicação $\varphi: x \mapsto c_{n} \varphi_{1}\left(\|x\|_{E_{n}}^{n}\right)$ está em $\mathcal{D}\left(E_{n}\right)$ e, portanto,

$$
I_{E}^{1}=I_{E_{n}}^{1}: \varphi_{1} \in \mathcal{A}_{0}(\mathbb{R}) \mapsto \varphi=c_{n}\left(\varphi_{1} \circ\|\cdot\|_{E_{n}}^{n}\right) \in \mathcal{D}\left(E_{n}\right)
$$


define uma aplicação injetora.

Definição 1.1.3. Para todo espaço normado, suave $E_{n}$ e para todo $q \in \mathbb{N}$ definimos

$$
\mathcal{A}_{q}\left(E_{n}\right):=I_{E_{n}}^{1}\left(\mathcal{A}_{q}(\mathbb{R})\right) .
$$

Proposição 1.1.4. Se $E=E_{n}$ é um espaço normado, suave, $\varphi \in \mathcal{A}_{q}\left(E_{n}\right)$ e $P \in \mathcal{P}_{k}(E, \mathbb{K})$ (o espaço dos polinômios $k$-homogêneos sobre $E$ ) com $1 \leq k \leq q$, então

$$
\int_{E_{n}} \varphi(x) \mathrm{d} x=1 e \int_{E_{n}} P(x) \varphi(x) \mathrm{d} x=0, \text { para } q \geq n
$$

Prova. Ver [12], (pg. 34) ou [2], (pg. 80).

Para uma lista de propriedades do conjunto $\mathcal{A}_{q}$ indicamos os trabalhos de Aragona e Biagioni [2], ou Kunzinger [12]. É a partir desta lista de propriedades (ver, por exemplo, a Proposição 1.7, Seção 1 em [2]) que podemos escrever simplesmente $\mathcal{A}_{q}(\mathbb{K}), q \in \mathbb{N}$ ao invés de $\mathcal{A}_{q}(E ; \mathbb{K}), q \in \mathbb{N}$, i.e., este conjunto não depende do espaço normado, suave $E$ e $\operatorname{sim}$ do corpo $\mathbb{K}$.

Definição 1.1.5. Seja $\mathcal{E}_{f}(\mathbb{K})$ o anel (operações ponto a ponto) das funções

$$
v: \mathcal{A}_{0}(\mathbb{K}) \rightarrow \mathbb{K}
$$

Então definimos $\mathcal{E}_{f}^{M}(\mathbb{K})$ o subanel de $\mathcal{E}_{f}(\mathbb{K})$ cujas funções v satisfazem:

(M) $\exists p \in \mathbb{N}$ tal que $\forall \varphi \in \mathcal{A}_{p}(\mathbb{K}), \exists C=C_{\varphi}>0, \exists \eta=\eta_{\varphi}>0$ tal que

$$
\left|v\left(\varphi_{\varepsilon}\right)\right| \leq C \varepsilon^{-p}, \forall 0<\varepsilon<\eta .
$$

Estas funções são ditas "moderadas".

Seja

$$
\Gamma:=\left\{\gamma: \mathbb{N} \rightarrow \mathbb{R}_{+} \mid \gamma(n)<\gamma(n+1), \forall n \in \mathbb{N} e \lim _{n \rightarrow \infty} \gamma(n)=\infty\right\}
$$

$o$ conjunto das seqüências estritamente crescentes que divergem quando $n \rightarrow \infty$. Então definimos $\mathcal{N}_{f}(\mathbb{K})$ o ideal de $\mathcal{E}_{f}^{M}(\mathbb{K})$ cujas funções v satisfazem:

$(N) \exists p \in \mathbb{N}, \exists \gamma \in \Gamma$ tal que $\forall q \geq p e \forall \varphi \in \mathcal{A}_{q}(\mathbb{K}), \exists C=C_{\varphi}>0, \exists \eta=\eta_{\varphi}>0$ tal que

$$
\left|v\left(\varphi_{\varepsilon}\right)\right| \leq C \varepsilon^{\gamma(q)-p}, \forall 0<\varepsilon<\eta
$$


A estas funções damos o nome de "nulas".

Então o anel dos números generalizados plenos de Colombeau é definido por

$$
\overline{\mathbb{K}}_{f}:=\mathcal{E}_{f}^{M}(\mathbb{K}) / \mathcal{N}_{f}(\mathbb{K})
$$

O exemplo abaixo nos mostra que $\overline{\mathbb{K}}_{f}$ não é um corpo .

Exemplo 1.1.6. Defina as funções $v, u: \mathcal{A}_{0}(\mathbb{K}) \rightarrow \mathbb{K}$ por

$$
\begin{aligned}
& v(\varphi):= \begin{cases}0, & \text { se } l(\varphi)=\frac{1}{n} \\
1, & \text { c.c. }\end{cases} \\
& u(\varphi):= \begin{cases}1, & \text { se } l(\varphi)=\frac{1}{n} \\
0, & \text { c.c. },\end{cases}
\end{aligned}
$$

onde para cada $\varphi \in \mathcal{A}_{0}(\mathbb{K})$ denotamos $l(\varphi):=\sup \{\mid x \| \varphi(x) \neq 0\}$. (ver [12]; final da pg. 37). É fácil ver que que $(u(\varphi))_{\varphi},(v(\varphi))_{\varphi} \in \mathcal{E}_{f}^{M}(\mathbb{K}) e(u(\varphi))_{\varphi},(v(\varphi))_{\varphi} \notin \mathcal{N}_{f}(\mathbb{K})$, portanto $\operatorname{cl}\left[(u(\varphi))_{\varphi}\right] \neq 0$ e $\operatorname{cl}\left[(v(\varphi))_{\varphi}\right] \neq 0$ em $\overline{\mathbb{K}}_{f}$. Mas $(u . v)(\varphi)=0, \forall \varphi$, i.e., u.v $=0$ em $\overline{\mathbb{K}}_{f}$.

$\mathbb{K}$ está contido em $\overline{\mathbb{K}}_{f}$ via a imersão constante

$$
\mathbb{K} \ni C \mapsto \operatorname{cl}\left[(C)_{\varphi}\right] \in \overline{\mathbb{K}}_{f}
$$

onde $C(\varphi)=C, \forall \varphi \in \mathcal{A}_{0}(\mathbb{K})$.

Definição 1.1.7. Um elemento $v \in \overline{\mathbb{K}}_{f}$ é associado ao zero e denotamos por $v \approx 0$ se para um (e, portanto, para todos) representante $(s)(v(\varphi))_{\varphi}$ de $v$ temos que

$$
\exists p \in \mathbb{N} \text { tal que } \lim _{\varepsilon \downarrow 0} v\left(\varphi_{\varepsilon}\right)=0, \forall \varphi \in \mathcal{A}_{p}(\mathbb{K}) .
$$

Dizemos que $v_{1} \approx v_{2}$ se $\left(v_{1}-v_{2}\right) \approx 0$. Se existe $a \in \mathbb{K}$ tal que $v \approx a$ então a é chamado de número associado ou sombra de $v$.

Seja $\Omega \subset \mathbb{R}^{n}$ um aberto e $\varphi \in \mathcal{D}(\Omega)$, o espaço das funções $\mathrm{C}^{\infty}(\Omega)$ com suporte compacto sobre $\Omega$. Denotamos por $i(\varphi)$ o diâmetro do suporte da $\varphi$, i.e.,

$$
i(\varphi):=\operatorname{diam}(\operatorname{supp}(\varphi)) .
$$

Notemos que

$$
i\left(\varphi_{\varepsilon}\right)=\varepsilon i(\varphi)
$$


para cada $\varepsilon>0$. Para $r \in \mathbb{R}$ definimos

$$
\dot{\alpha}_{r}: \mathcal{A}_{0}(\mathbb{K}) \rightarrow \mathbb{R}
$$

por

$$
\dot{\alpha}_{r}(\varphi):=(i(\varphi))^{r}
$$

Notemos que

$$
\dot{\alpha}_{r}\left(\varphi_{\varepsilon}\right)=\varepsilon^{r} \dot{\alpha}_{r}(\varphi) .
$$

Lembrando que para $\overline{\mathbb{K}}$, o anel dos números generalizados simplificados de Colombeau, Aragona e Juriaans [5] definiram para $r \in \mathbb{R}$ a aplicação

$$
\alpha_{r}: I \rightarrow \mathbb{R}
$$

dada por

$$
\alpha_{r}(\varepsilon):=\varepsilon^{r},
$$

temos por (1.2) que

$$
\dot{\alpha}_{r}\left(\varphi_{\varepsilon}\right)=\alpha_{r}(\varepsilon) \dot{\alpha}_{r}(\varphi) .
$$

Definição 1.1.8. Para $x \in \overline{\mathbb{K}}_{f}$ defina

$$
\mathrm{A}(x):=\left\{r \in \mathbb{R} \mid \dot{\alpha}_{-r} x \approx 0\right\}
$$

e a valuação de $x$ por

$$
\mathrm{V}(x):=\sup (\mathrm{A}(x))
$$

O lema que segue caracteriza um elemento pertencente a $\mathrm{A}(x), x \in \overline{\mathbb{K}}_{f}$, mais precisamente temos:

Lema 1.1.9. Seja $x \in \overline{\mathbb{K}}_{f}$. Então $r \in \mathrm{A}(x)$ se, e somente se, existe $p \in \mathbb{N}$ tal que

$$
\lim _{\varepsilon \downarrow 0} \varepsilon^{-r} x\left(\varphi_{\varepsilon}\right)=0, \forall \varphi \in \mathcal{A}_{p}(\mathbb{K}) .
$$

Prova. De fato, $r \in \mathrm{A}(x)$ se, e somente se, $\dot{\alpha}_{-r} x \approx 0$ se, e somente se, existe $p \in \mathbb{N}$ tal que

$$
\lim _{\varepsilon \downarrow 0} \dot{\alpha}_{-r}\left(\varphi_{\varepsilon}\right) x\left(\varphi_{\varepsilon}\right)=0, \forall \varphi \in \mathcal{A}_{p}(\mathbb{K})
$$


se, e somente se, existe $p \in \mathbb{N}$ tal que

$$
\lim _{\varepsilon \downarrow 0} \varepsilon^{-r} x\left(\varphi_{\varepsilon}\right)=0, \forall \varphi \in \mathcal{A}_{p}(\mathbb{K}),
$$

pois $\dot{\alpha}_{-r}\left(\varphi_{\varepsilon}\right)=i(\varphi)^{-r} \varepsilon^{-r}$ e $i(\varphi) \neq 0, \varphi \in \mathcal{A}_{p}(\mathbb{K})$. E, portanto, segue o resultado.

A proposição seguinte enumera algumas propriedades de $\mathrm{V}(x), x \in \overline{\mathbb{K}}_{f}$. Propriedades estas que permitem definir uma função $D: \overline{\mathbb{K}}_{f} \times \overline{\mathbb{K}}_{f} \rightarrow \mathbb{R}_{+}$que define uma ultra-métrica sobre $\overline{\mathbb{K}}_{f}$ que é invariante por translação Corolário 1.1.11.

Proposição 1.1.10. Para $x, y \in \mathcal{E}_{f}^{M}(\mathbb{K})$ e $\lambda \in \mathbb{K}$, temos:

(i) $\mathrm{V}(\lambda x)=\mathrm{V}(x)$ se $\lambda \neq 0$;

(ii) $\mathrm{V}(x y) \geq \mathrm{V}(x)+\mathrm{V}(y)$;

(iii) $\mathrm{V}\left(\dot{\alpha}_{r} x\right)=r+\mathrm{V}(x)$

(iv) $\mathrm{V}(x+y) \geq \inf \{\mathrm{V}(x), \mathrm{V}(y)\}$;

(v) $\mathrm{V}(x)=+\infty \Leftrightarrow x \in \mathcal{N}_{f}(\mathbb{K})$

(vi) $\mathrm{V}$ é constante sobre cada classe de equivalência módulo $\mathcal{N}_{f}(\mathbb{K})$. Isto é,

$$
\mathrm{V}(x)=\mathrm{V}(x+y), \forall x \in \mathcal{E}_{f}^{M}(\mathbb{K}) \forall y \in \mathcal{N}_{f}(\mathbb{K})
$$

Prova. (i) Basta mostrarmos que $\mathrm{A}(\lambda x)=\mathrm{A}(x)$. Seja $r \in \mathrm{A}(\lambda x)$. Então

$$
\dot{\alpha}_{-r}(\lambda x) \approx 0 \Rightarrow\left(\dot{\alpha}_{-r} \lambda\right) x \approx 0 \Rightarrow\left(\lambda \dot{\alpha}_{-r}\right) x \approx 0 \Rightarrow \lambda\left(\dot{\alpha}_{-r} x\right) \approx 0
$$

Como $\lambda \neq 0$ segue que $\lambda$ não está associado com zero e, portanto,

$$
\dot{\alpha}_{-r} x \approx 0 \Rightarrow r \in \mathrm{A}(x) \text {. }
$$

Logo, $\mathrm{A}(\lambda x) \subseteq \mathrm{A}(x)$. Agora, se $r \in \mathrm{A}(x)$, então $\dot{\alpha}_{-r} x \approx 0$. Portanto, multiplicando ambos os membros por $\lambda \neq 0$, vem

$$
\lambda\left(\dot{\alpha}_{-r} x\right) \approx 0 \Rightarrow\left(\lambda \dot{\alpha}_{-r}\right) x \approx 0 \Rightarrow\left(\dot{\alpha}_{-r} \lambda\right) x \approx 0 \Rightarrow \dot{\alpha}_{-r}(\lambda x) \approx 0 \Rightarrow r \in \mathrm{A}(\lambda x) .
$$

$\mathrm{E}$, portanto, $\mathrm{A}(x) \subseteq \mathrm{A}(\lambda x)$. Das duas inclusões segue o resultado. 
(ii) Seja $\mathrm{A}(x)+\mathrm{A}(y):=\{r \in \mathbb{R} \mid r=a+b$, onde $a \in \mathrm{A}(x)$ e $b \in \mathrm{A}(y)\}$. Então, para mostrarmos que $\mathrm{V}(x)+\mathrm{V}(y) \leq \mathrm{V}(x y)$ basta mostrarmos que $\mathrm{A}(x)+\mathrm{A}(y) \subseteq \mathrm{A}(x y)$. Ora, se $r \in \mathrm{A}(x)+\mathrm{A}(y)$, então $r=a+b$, onde $a \in \mathrm{A}(x)$ e $b \in \mathrm{A}(y)$. Assim, temos que $\dot{\alpha}_{-a} x \approx 0$ e $\dot{\alpha}_{-b} y \approx 0$. Logo, multiplicando membro a membro estas duas equações, vem

$$
\begin{aligned}
\left(\dot{\alpha}_{-a} x\right)\left(\dot{\alpha}_{-b} y\right) \approx 0 & \Rightarrow \dot{\alpha}_{-a}\left(x \dot{\alpha}_{-b}\right) y \approx 0 \\
& \Rightarrow \dot{\alpha}_{-a}\left(\dot{\alpha}_{-b} x\right) y \approx 0 \\
& \Rightarrow\left(\dot{\alpha}_{-a} \dot{\alpha}_{-b}\right)(x y) \approx 0 \\
& \Rightarrow \dot{\alpha}_{-(a+b)}(x y) \approx 0 \\
& \Rightarrow \dot{\alpha}_{-r}(x y) \approx 0 \\
& \Rightarrow r \in \mathrm{A}(x y) .
\end{aligned}
$$

Portanto, $\mathrm{A}(x)+\mathrm{A}(y) \subseteq \mathrm{A}(x y)$.

(iii) Com efeito, se $a \in \mathrm{A}\left(\dot{\alpha}_{r} x\right)$, então

$$
\dot{\alpha}_{-a}\left(\dot{\alpha}_{r} x\right) \approx 0 \Rightarrow\left(\dot{\alpha}_{-a} \dot{\alpha}_{r}\right) x \approx 0 \Rightarrow \dot{\alpha}_{-(a-r)} x \approx 0 \Rightarrow(a-r) \in \mathrm{A}(x)
$$

Logo, existe $b \in \mathrm{A}(x)$ tal que $b=a-r \Rightarrow a=r+b$. Portanto,

$$
\sup \left(\mathrm{A}\left(\dot{\alpha}_{r} x\right)\right)=r+\sup (\mathrm{A}(x)) \Rightarrow \mathrm{V}\left(\dot{\alpha}_{r} x\right)=r+\mathrm{V}(x)
$$

(iv): Seja $r<\inf \{\mathrm{V}(x), \mathrm{V}(y)\}$. Então $r<\mathrm{V}(x)$ e $r<\mathrm{V}(y)$. Assim, $r \in \mathrm{A}(x)$ e $r \in \mathrm{A}(y)$. Pelo Lema 1.1.9, temos que existe $p \in \mathbb{N}$ tal que

$$
\lim _{\varepsilon \downarrow 0} \varepsilon^{-r} x\left(\varphi_{\varepsilon}\right)=0=\lim _{\varepsilon \downarrow 0} \varepsilon^{-r} y\left(\varphi_{\varepsilon}\right), \forall \varphi \in \mathcal{A}_{p}(\mathbb{K})
$$

Agora, para este $p \in \mathbb{N}$ temos que

$$
\lim _{\varepsilon \downarrow 0} \varepsilon^{-r}\left(x\left(\varphi_{\varepsilon}\right)+y\left(\varphi_{\varepsilon}\right)\right)=0, \forall \varphi \in \mathcal{A}_{p}(\mathbb{K})
$$

E, novamente, pelo Lema 1.1.9 podemos concluir que $r \in \mathrm{A}(x+y)$ e, portanto,

$$
r \leq \sup (\mathrm{A}(x+y))=\mathrm{V}(x+y)
$$

o que mostra que $\mathrm{V}(x+y) \geq \inf \{V(x), \mathrm{V}(y)\}$.

$(v) \mathrm{V}(x)=+\infty \Leftrightarrow \mathrm{A}(x)=\mathbb{R} \Leftrightarrow \dot{\alpha}_{-r} x \approx 0, \forall r \in \mathbb{R} \Leftrightarrow \operatorname{cl}[x]=\operatorname{cl}[0] \Leftrightarrow x \in \mathcal{N}_{f}(\mathbb{K})$. 
(vi) Sabemos de $(i v)$ que $\mathrm{V}(x+y) \geq \inf \{\mathrm{V}(x), \mathrm{V}(y)\}=\mathrm{V}(x)$, pois $y \in \mathcal{N}_{f}(\mathbb{K})$ e por $(v)$ temos que $\mathrm{V}(y)=+\infty$. Portanto, $\mathrm{V}(x+y) \geq \mathrm{V}(x)$. Por outro lado, se $a \in \mathrm{A}(x+y)$, então $\dot{\alpha}_{-a}(x+y) \approx 0 \Rightarrow \dot{\alpha}_{-a} x+\dot{\alpha}_{-a} y \approx 0 \Rightarrow \dot{\alpha}_{-a} x \approx 0$ e $\dot{\alpha}_{-a} y \approx 0^{1} \Rightarrow a \in \mathrm{A}(x) \Rightarrow$ $\mathrm{A}(x+y) \subset \mathrm{A}(x), \forall y \in \mathcal{N}_{f}(\mathbb{K}) \Rightarrow \mathrm{V}(x+y) \leq \mathrm{V}(x)$. Das duas desigualdades acima concluímos o que queríamos.

A proposição acima mostra que a função

$$
D: \overline{\mathbb{K}}_{f} \times \overline{\mathbb{K}}_{f} \rightarrow \mathbb{R}_{+}
$$

definida por

$$
D(x, y):=e^{-V(x-y)}
$$

está bem definida e com esta notação temos o seguinte:

Corolário 1.1.11. A função $D$ é uma ultra-métrica sobre $\overline{\mathbb{K}}_{f}$ que é invariante por translação.

Prova. Parte 1: Para mostrarmos que $D$ é uma ultra-métrica precisamos mostrar que para todo $x, y, w \in \overline{\mathbb{K}}_{f}$ vale:

(a) $D(x, y) \geq 0$ e $D(x, y)=0$ se, e somente se, $x-y \in \mathcal{N}_{f}$, isto é, $\operatorname{cl}[x]=\operatorname{cl}[y]$

(b) $D(x, y)=D(y, x)$;

(c) $D(x, y) \leq \max \{D(x, y), D(y, w)\}$

Vamos mostrar $(a),(b)$ e $(c)$ :

(a) Temos $D(x, y)=e^{-\mathrm{V}(x-y)} \geq 0$ e $D(x, y)=0 \Leftrightarrow e^{-\mathrm{V}(x-y)}=0 \Leftrightarrow \mathrm{V}(x-y)=+\infty \Leftrightarrow$ $x-y \in \mathcal{N}_{f} \Leftrightarrow \operatorname{cl}[x]=\operatorname{cl}[y]$ (ver Proposição 1.1.10(v)).

(b) $\operatorname{Temos} D(x, y)=e^{-\mathrm{V}(x-y)}=e^{-\mathrm{V}(-1(y-x))}=e^{-\mathrm{V}(y-x)}=D(y, x)$, pois $\mathrm{V}(-1(y-x))=$ $\mathrm{V}(y-x)$ (ver Proposição 1.1.10(i) para $\lambda=-1$ ).

(c) Temos que $x-y=(x-w)+(w-y)$, assim

$$
\begin{aligned}
\mathrm{V}(x-y) & =\mathrm{V}((x-w)+(w-y)) \text { (ver proposição 1.1.10(iv)) } \\
& \geq \inf \{\mathrm{V}(x-w), \mathrm{V}(w-y)\}
\end{aligned}
$$

${ }^{1}$ Lembremos que $\dot{\alpha}_{-a} y \in \mathcal{N}_{f}(\mathbb{K})$, pois $\mathcal{N}_{f}(\mathbb{K})$ é um ideal de $\mathcal{E}_{f}^{M}(\mathbb{K})$ 


$$
\begin{aligned}
& \text { Logo, }-\mathrm{V}(x-y) \leq \sup \{-\mathrm{V}(x-w),-\mathrm{V}(w-y)\} \text { e, portanto, } \\
& \qquad \begin{aligned}
D(x, y)= & e^{-\mathrm{V}(x-y)} \\
\leq & e^{\sup \{-\mathrm{V}(x-w),-\mathrm{V}(w-y)\}} \\
\leq & \sup \left\{e^{-\mathrm{V}(x-w)}, e^{-\mathrm{V}(w-y)}\right\} \\
\leq & \sup \{D(x, w), D(w, y)\} \\
& =\max \{D(x, w), D(w, y)\} \therefore D(x, y) \leq \max \{D(x, w), D(w, y)\} .
\end{aligned}
\end{aligned}
$$

De $(a),(b)$ e $(c)$ segue que $D$ é uma ultra-métrica.

Parte 2: Agora, vamos mostrar que $D$ é invariante por translação. De fato, para todo $x, y, z \in \overline{\mathbb{K}}_{f}$, temos

$$
\begin{aligned}
D(x+z, y+z) & =e^{-\mathrm{V}((x+z)-(y+z))} \\
& =e^{-\mathrm{V}(x+(z-y)-z)} \\
& =e^{-\mathrm{V}((x-y)+(z-z))} \\
& =e^{-\mathrm{V}(x-y+0)} \\
& =e^{-\mathrm{V}(x-y)} \\
& =D(x, y) .
\end{aligned}
$$

E, portanto,

$$
D(x+z, y+z)=D(x, y), \forall x, y, z \in \overline{\mathbb{K}}_{f}
$$

o que mostra a invariância por translação.

$D$ determina uma estrutura uniforme sobre $\overline{\mathbb{K}}_{f}$ chamada de estrutura uniforme escalar cortante sobre $\overline{\mathbb{K}}_{f}$ e a topologia resultante de $D$ é chamada a topologia escalar cortante sobre $\overline{\mathbb{K}}_{f}$ e a denotaremos por $\tau_{s f}$.

Seja $x \in \overline{\mathbb{K}}_{f}$ e $r \in \mathbb{R}_{+}^{*}$. Então de agora em diante denotamos por $B_{r}(x)$ (resp. $B_{r}^{\prime}(x)$ e $S_{r}(x)$ ) a $D$-bola aberta (resp. $D$-bola fechada e $D$-esfera) com centro em $x$ e raio $r$. No caso onde $x=0$ omitiremos na notação escrevendo simplesmente $B_{r}$ (resp. $B_{r}^{\prime}$ e $S_{r}$ ). Para simplificar definimos

$$
\|x\|:=D(x, 0), \forall x \in \overline{\mathbb{K}}_{f}
$$

e a distância entre dois elementos $x, y \in \overline{\mathbb{K}}_{f}$ por

$$
d(x, y):=\|x-y\| .
$$


O resultado abaixo é uma conseqüência imediata da Proposição 1.1 .10 e da definição de $D$.

Corolário 1.1.12. Para $x, y \in \overline{\mathbb{K}}_{f}, r \in \mathbb{R}, s \in \mathbb{R}_{+}^{*}$ e $a, b \in \mathbb{K}$, temos

(i) $\|x+y\| \leq \max \{\|x\|,\|y\|\}$ e $\|x y\| \leq\|x\|\|y\|$;

(ii) $\|x\|=0 \Leftrightarrow \operatorname{cl}[x]=\operatorname{cl}[0]$;

(iii) $\|a x\|=\|x\|$, se $a \neq 0$;

(iv) $\left\|\dot{\alpha}_{r} x\right\|=e^{-r}\|x\| ;$

(v) $\|a\|=1$, se $a \neq 0$;

(vi) $\|a-b\|=1-\delta_{a b}(\delta$ de Kronecker $)$.

Prova. (i) Parte 1: Com efeito, se $\mathrm{V}(x) \leq \mathrm{V}(y)$, então

$$
e^{\mathrm{V}(x)} \leq e^{\mathrm{V}(y)} \Rightarrow e^{-\mathrm{V}(x)} \geq e^{-\mathrm{V}(y)}
$$

mas então $\|x\| \geq\|y\|$. Neste caso temos que $\max \{\|x\|,\|y\|\}=\|x\|$. Agora, pela Proposição $1.1 .10(i v)$, vem

$$
\begin{aligned}
\|x+y\| & =e^{-\mathrm{V}(x+y)} \\
& \leq e^{-\mathrm{V}(x)} \\
& =\|x\| \\
& =\max \{\|x\|,\|y\|\} .
\end{aligned}
$$

Portanto, $\|x+y\| \leq \max \{\|x\|,\|y\|\}$.

Parte 2: Pela Proposição 1.1.10 (ii), temos

$$
\begin{aligned}
\|x y\| & =e^{-\mathrm{V}(x y)} \\
& \leq e^{-(\mathrm{V}(x)+\mathrm{V}(y))} \\
& =e^{-\mathrm{V}(x)-\mathrm{V}(y)} \\
& =e^{-\mathrm{V}(x)} e^{-\mathrm{V}(y)} \\
& =\|x\|\|y\| .
\end{aligned}
$$


Portanto, $\|x y\| \leq\|x\|\|y\|$.

(ii) Pela Proposição 1.1.10 $(v)$, temos

$$
\|x\|=0 \Leftrightarrow e^{-\mathrm{V}(x)}=0 \Leftrightarrow \mathrm{V}(x)=+\infty \Leftrightarrow x \in \mathcal{N}_{f}(\mathbb{K}) \Leftrightarrow x=0 .
$$

Logo, $\|x\|=0 \Leftrightarrow \operatorname{cl}[x]=\operatorname{cl}[0]$.

(iii) Temos, se $a \neq 0$ que

$$
\begin{aligned}
\|a x\| & =e^{-\mathrm{V}(a x)}(\text { por }(i) \text { da Proposição } 1.1 .10, \text { temos }) \\
& =e^{-\mathrm{V}(x)} \\
& =\|x\|
\end{aligned}
$$

isto é, $\|a x\|=\|x\|$.

(iv) Temos pela Proposição 1.1.10 (iii) que

$$
\begin{aligned}
\left\|\dot{\alpha}_{r} x\right\| & =e^{-\mathrm{V}\left(\dot{\alpha}_{r} x\right)} \\
& =e^{-(r+\mathrm{V}(x))} \\
& =e^{-r-\mathrm{V}(x)} \\
& =e^{-r} e^{-\mathrm{V}(x)} \\
& =e^{-r}\|x\| .
\end{aligned}
$$

Portanto, $\left\|\dot{\alpha}_{r} x\right\|=e^{-r}\|x\|$.

(v) Com efeito, se $a \neq 0$ e $r \in \mathrm{A}(a)$, então $\dot{\alpha}_{-r} a \approx 0 \Rightarrow \dot{\alpha}_{-r} \approx 0 \Rightarrow r<0$. Assim, $\mathrm{A}(a)=]-\infty, 0[$ e, portanto, $\mathrm{V}(a)=\sup (\mathrm{A}(a))=\sup (]-\infty, 0[)=0$. Logo, $\|a\|=e^{-\mathrm{V}(a)}=e^{0}=1$, isto é, $\|a\|=1$.

(vi) Se $a=b$, então $a-b=0$ e por $(i i)$, temos que $\|a-b\|=0$. Agora, se $a \neq b$, então $c=a-b \neq 0, c \in \mathbb{K}$ e, por $(v)$, temos que $\|c\|=1$. Logo,

$$
\|a-b\|= \begin{cases}0, & \text { se } a=b \\ 1, & \text { se } a \neq b\end{cases}
$$

E pela definição do $\delta$ de Kronecker, abaixo

$$
\delta_{a b}:= \begin{cases}1, & \text { se } a=b \\ 0, & \text { se } a \neq b .\end{cases}
$$

segue que $\|a-b\|=1-\delta_{a b}$. 
Proposição 1.1.13. $\left(\overline{\mathbb{K}}_{f}, \tau_{s f}\right)$ é um anel topológico completo.

Prova. Parte 1: Como $\overline{\mathbb{K}}_{f}$ é um anel quociente, basta mostrarmos que as aplicações $(+): \overline{\mathbb{K}}_{f} \times \overline{\mathbb{K}}_{f} \rightarrow \overline{\mathbb{K}}_{f}$ tal que $(x, y) \mapsto x+y$ e $(\cdot): \overline{\mathbb{K}}_{f} \times \overline{\mathbb{K}}_{f} \rightarrow \overline{\mathbb{K}}_{f}$ tal que $(x, y) \mapsto x \cdot y$, isto é, a soma e a multiplicação em $\overline{\mathbb{K}}_{f}$, respectivamente, são contínuas. Sabendo-se que $\left(\overline{\mathbb{K}}_{f}, \tau_{s f}\right)$ é uma topologia ultra-métrica, é suficiente mostrarmos que se $\left\{x_{n}\right\}_{n \in \mathbb{N}}$ e $\left\{y_{n}\right\}_{n \in \mathbb{N}}$ são duas seqüências em $\overline{\mathbb{K}}_{f}$ tais que $x_{n} \underset{n \rightarrow \infty}{\longrightarrow} x$ e $y_{n} \underset{n \rightarrow \infty}{\longrightarrow} y$, então $\left(x_{n}+y_{n}\right) \underset{n \rightarrow \infty}{\longrightarrow}(x+y)$ e $\left(x_{n} \cdot y_{n}\right) \underset{n \rightarrow \infty}{\longrightarrow}(x \cdot y)$. Ora,

$$
\begin{aligned}
\left\|\left(x_{n}+y_{n}\right)-(x+y)\right\| & =\left\|\left(x_{n}-x\right)+\left(y_{n}-y\right)\right\| \text { (pela Corolário 1.1.12(i), temos) } \\
& \leq \max \left\{\left\|x_{n}-x\right\|,\left\|y_{n}-y\right\|\right\}
\end{aligned}
$$

e esta última tende a zero quando $n \longrightarrow \infty$, pois $\left\|x_{n}-x\right\| \underset{n \rightarrow \infty}{\longrightarrow} 0$ e $\left\|y_{n}-y\right\| \underset{n \rightarrow \infty}{\longrightarrow} 0$. Portanto,

$$
\left\|\left(x_{n}+y_{n}\right)-(x+y)\right\| \underset{n \rightarrow \infty}{\longrightarrow} 0 \Rightarrow\left(x_{n}+y_{n}\right) \underset{n \rightarrow \infty}{\longrightarrow}(x+y) .
$$

$\mathrm{E}$ isto mostra a continuidade da operação de soma (+).

Agora, vamos mostrar a continuidade da operação de multiplicação (·). Ora,

$$
\begin{aligned}
\left\|x_{n} \cdot y_{n}-x \cdot y\right\| & =\left\|x_{n}\left(y_{n}-y\right)+y\left(x_{n}-x\right)\right\| \text { (pelo Corolário 1.1.12(i), temos) } \\
& \leq \max \left\{\left\|x_{n}\left(y_{n}-y\right)\right\|,\left\|y\left(x_{n}-x\right)\right\|\right\} \text { (pelo Corolário 1.1.12(i), temos) } \\
& \leq \max \left\{\left\|x_{n}\right\|\left\|y_{n}-y\right\|,\|y\|\left\|x_{n}-x\right\|\right\}
\end{aligned}
$$

e esta última tende a zero quando $n \longrightarrow \infty$, pois

$$
\left\|x_{n}-x\right\| \underset{n \rightarrow \infty}{\longrightarrow} 0,\left\|y_{n}-y\right\| \underset{n \rightarrow \infty}{\longrightarrow} 0
$$

e $\left\|x_{n}\right\|=\left\|\left(x_{n}-x\right)+x\right\| \leq \max \left\{\left\|x_{n}-x\right\|,\|x\|\right\} \underset{n \rightarrow \infty}{\longrightarrow}\|x\|=$ const $\Rightarrow\left\|x_{n}\right\| \leq$ const. Portanto,

$$
\left\|x_{n} \cdot y_{n}-x \cdot y\right\| \underset{n \rightarrow \infty}{\longrightarrow} 0 \Rightarrow\left(x_{n} \cdot y_{n}\right) \underset{n \rightarrow \infty}{\longrightarrow}(x \cdot y)
$$

o que mostra a continuidade da multiplicação (·). Assim, concluímos que $\left(\overline{\mathbb{K}}_{f}, \tau_{s, f}\right)$ é um anel topológico.

Parte 2: Segue da completude das álgebras $\mathcal{G}_{f}(\bar{\Omega})$ e $\mathcal{G}_{f}(\Omega)$ (ver Capítulo 3, Teorema 3.4.13 e Corolário 3.4.14) e do fato que $\overline{\mathbb{K}}_{f}$ é o anel das constantes de tais álgebras.

Proposição 1.1.14. (i) $\left(\overline{\mathbb{K}}_{f}, \tau_{s f}\right)$ não é um $\mathbb{K}$-espaço vetorial topológico. 
(ii) $\left(\overline{\mathbb{K}}_{f}, \tau_{s f}\right)$ não é separável.

(iii) $\left(\overline{\mathbb{K}}_{f}, \tau_{s f}\right)$ não é localmente compacto.

Prova. (i) Para mostrarmos isto, mostraremos que a multiplicação usual

$$
M: \mathbb{K} \times \overline{\mathbb{K}}_{f} \rightarrow \overline{\mathbb{K}}_{f}
$$

dada por

$$
M(a, y)=a y
$$

onde $\mathbb{K}$ está munido com sua topologia usual não é contínua. Isto é, existe

$$
\left(a_{n}, y_{n}\right) \underset{n \rightarrow \infty}{\longrightarrow}(a, y)
$$

mas

$$
M\left(a_{n}, y_{n}\right) \underset{n \rightarrow \infty}{\nrightarrow} M(a, y) \text {, i.e., } a_{n} y_{n} \underset{n \rightarrow \infty}{\nrightarrow} a y .
$$

De fato, basta tomarmos uma seqüência $\left\{a_{n}\right\}_{n \in \mathbb{N}} \subset \mathbb{K}$ com $a_{n} \underset{n \rightarrow \infty}{\longrightarrow} 0$ (na topologia usual de $\mathbb{K})$ e $a_{n} \neq 0, \forall n \in \mathbb{N}$ e uma seqüência $\left\{y_{n}\right\}_{n \in \mathbb{N}} \operatorname{com} y_{n}=y \neq 0, \forall n \in \mathbb{N}$. Então temos que

$$
\left(a_{n}, y_{n}\right) \underset{n \rightarrow \infty}{\longrightarrow}(0, y)
$$

mas

$$
M\left(a_{n}, y_{n}\right) \underset{n \rightarrow \infty}{\nrightarrow} M(0, y)=0,
$$

pois pelo Corolário 1.1.12-(iii), temos $\left\|M\left(a_{n}, y_{n}\right)\right\|=\left\|a_{n} y_{n}\right\|=\left\|a_{n} y\right\|=\|y\| \neq 0$, já que $y \neq 0$.

As afirmações (ii) e (iii) são justificadas na Seção 1.3, Proposição 1.3.5 (ii).

\subsection{Propriedades algébricas de $\overline{\mathbb{K}}_{f}$}

Nesta seção enunciamos e provamos algumas propriedades algébricas do anel dos números generalizados plenos de Colombeau. Na verdade procuramos estender alguns dos resultados algébricos obtidos para o anel dos números generalizados simplificados de Colombeau $\overline{\mathbb{K}}$ em [5], para o anel dos números generalizados plenos de Colombeau $\overline{\mathbb{K}}_{f}$, objeto de nossos estudos. Damos início com o seguinte: 
Lema 1.2.1. (i) $x \in B_{1}$ se, e somente se, $\mathrm{V}(x)>0$.

(ii) Se $x \in B_{1}$, então vale:

(a) $x \approx 0$;

(b) $D(1, x)=\|1-x\| \geq 1$. Portanto, $1 \notin \overline{B_{1}}, B_{1} \cap B_{1}(1)=\emptyset, B_{1}^{\prime} \supset \overline{B_{1}}$ e $B_{1}^{\prime} \neq \overline{B_{1}}$.

Prova. (i) Temos que

$$
\begin{aligned}
x \in B_{1} & \Leftrightarrow\|x\|<1 \\
& \Leftrightarrow e^{-\mathrm{V}(x)}<1 \\
& \Leftrightarrow e^{-\mathrm{V}(x)}<e^{0} \\
& \Leftrightarrow-\mathrm{V}(x)<0 \\
& \Leftrightarrow \mathrm{V}(x)>0 .
\end{aligned}
$$

(ii)(a) Para mostrarmos que $x \approx 0$, precisamos mostrar que existe $p \in \mathbb{N}$ tal que

$$
\lim _{\varepsilon \rightarrow 0} x\left(\varphi_{\varepsilon}\right)=0, \forall \varphi \in \mathcal{A}_{p}(\mathbb{K}) .
$$

Seja $r \in \mathrm{A}(x)$. Então $\dot{\alpha}_{-r} x \approx 0$. Logo, existe $p^{\prime} \in \mathbb{N}$ tal que

$$
\lim _{\varepsilon \rightarrow 0} \dot{\alpha}_{-r}\left(\varphi_{\varepsilon}\right) x\left(\varphi_{\varepsilon}\right)=0, \forall \varphi \in \mathcal{A}_{p^{\prime}}(\mathbb{K})
$$

o que implica

$$
(i(\varphi))^{-r} \lim _{\varepsilon \rightarrow 0} \varepsilon^{-r} x\left(\varphi_{\varepsilon}\right)=0, \forall \varphi \in \mathcal{A}_{p^{\prime}}(\mathbb{K}) .
$$

Como $(i(\varphi))^{-r}>0, \forall \varphi \in \mathcal{A}_{p^{\prime}}(\mathbb{K})$. Segue que

$$
\lim _{\varepsilon \rightarrow 0} \varepsilon^{-r} x\left(\varphi_{\varepsilon}\right)=0, \forall \varphi \in \mathcal{A}_{p^{\prime}}(\mathbb{K}) .
$$

Logo, da definição da valuação de $x$ e de $(i)$ segue existe $p \in \mathbb{N}$ tal que

$$
\lim _{\varepsilon \rightarrow 0} \varepsilon^{-p} x\left(\varphi_{\varepsilon}\right)=0, \forall \varphi \in \mathcal{A}_{p}(\mathbb{K}) \Rightarrow \varepsilon^{-p} x\left(\varphi_{\varepsilon}\right)<1, \forall \varphi \in \mathcal{A}_{p}(\mathbb{K})
$$

e $\varepsilon$ suficientemente pequeno. Daí, $x\left(\varphi_{\varepsilon}\right)<\varepsilon^{p}, \forall \varphi \in \mathcal{A}_{p}(\mathbb{K})$ e $\varepsilon$ suficientemente pequeno.

$\mathrm{E}$, portanto, existe $p \in \mathbb{N}$ tal que

$$
\lim _{\varepsilon \rightarrow 0} x\left(\varphi_{\varepsilon}\right)=0, \forall \varphi \in \mathcal{A}_{p}(\mathbb{K})
$$


o que implica $x \approx 0$.

(b) Suponhamos, por absurdo, que $D(1, x)=\|1-x\|<1$, então $(1-x) \in B_{1}$. Por $(a)$, temos que $(1-x) \approx 0 \Rightarrow x \approx 1$ o que contradiz $(a)$ já que $x \in B_{1}$ e neste caso temos que $x \approx 0$. Logo, $D(1, x)=\|1-x\|=e^{-\mathrm{V}(1-x)} \geq 1$. Neste caso também temos que $\mathrm{V}(1-x) \leq 0$. Por último, se $1 \in \overline{B_{1}}$, então existe uma seqüência $\left\{x_{n}\right\}_{n \in \mathbb{N}}$ em $B_{1}$ que é $\tau_{s f}$-convergente a 1 e, portanto, $1 \leq D\left(1, x_{n}\right)=\left\|1-x_{n}\right\| \rightarrow 0$ quando $n \rightarrow \infty$ o que é um absurdo!

Proposição 1.2.2. Sejam $\left\{a_{n}\right\}_{n \in \mathbb{N}} \subset \mathbb{K}$ uma seqüência qualquer e $0 \neq x \in B_{1}$, i.e., $\|x\|<1$. Então a série

$$
\sum_{n \geq 0} a_{n} x^{n}
$$

converge em $\overline{\mathbb{K}}_{f}$. Em particular, se $a_{n}=1, \forall n \in \mathbb{N}$, então

$$
\sum_{n \geq 0} x^{n}
$$

converge e temos que

$$
(1-x) \sum_{n \geq 0} x^{n}=1
$$

$E$, portanto, $(1-x) \in \operatorname{Inv}\left(\overline{\mathbb{K}}_{f}\right)$.

Prova. Seja $\left\{S_{n}\right\}_{n \in \mathbb{N}} \subset \overline{\mathbb{K}}_{f}$ a seqüência de somas parciais,

$$
S_{n}=\sum_{k=0}^{n} a_{k} x^{k} .
$$

Como, pelo Teorema (1:1.13), $\left(\overline{\mathbb{K}}_{f}, \tau_{s f}\right)$ é um anel topológico completo segue que para mostrarmos a convergência da seqüência $\left\{S_{n}\right\}_{n \in \mathbb{N}}$, basta mostrarmos que é uma seqüência de Cauchy. De fato,

$$
\begin{aligned}
\left\|S_{m}-S_{n}\right\| & =\left\|\sum_{k=n+1}^{m} a_{k} x^{k}\right\| \text { (pelo Corolário 1.1.12(i), temos) } \\
& \leq \max _{n+1 \leq k \leq m}\left\{\left\|a_{k} x^{k}\right\|\right\}\left(a_{k} \in \mathbb{K} \text { e } a_{k} \neq 0, \text { Corolário } 1.1 .12(\text { iii }),\right. \text { temos) } \\
& =\max _{n+1 \leq k \leq m}\left\{\left\|x^{k}\right\|\right\} \\
& \leq \max _{n+1 \leq k \leq m}\left\{\|x\|^{k}\right\}\left(\|x\|<1, \text { pois } x \in B_{1}\right) \\
& \leq\|x\|^{n+1} \\
& =e^{-\mathrm{V}(x)(n+1)}
\end{aligned}
$$


$\mathrm{E}$, portanto, existe $n_{0} \in \mathbb{N}$ tal que para todo $m>n \geq n_{0}$ implica que

$$
\left\|S_{m}-S_{n}\right\| \leq e^{-\mathrm{V}(x)(n+1)}<e^{-\mathrm{V}(x) n_{0}} .
$$

Agora, como $x \in B_{1}$ segue pelo Lema 1.2.1 $(i)$ que $V(x)>0$ o que implica $V(x) n_{0}>0$. Daí, temos $e^{-\mathrm{V}(x) n_{0}} \underset{n \rightarrow \infty}{\longrightarrow} 0$ e, portanto, $\left\|S_{m}-S_{n}\right\| \underset{m, n \rightarrow \infty}{\longrightarrow} 0$. Logo, $\left\{S_{n}\right\}_{n \in \mathbb{N}}$ é uma seqüência de Cauchy e, portanto, $\left\{S_{n}\right\}_{n \in \mathbb{N}}$ é convergente em $\overline{\mathbb{K}}_{f}$. Em particular,

$$
\sum_{k=0}^{\infty} x^{k}
$$

converge. Isto é, existe $z \in \overline{\mathbb{K}}_{f}$ tal que $S_{n} \underset{n \rightarrow \infty}{\longrightarrow} z$, onde $\left\{S_{n}\right\}_{n \in \mathbb{N}}$ é a seqüência de somas parciais de (1.3). Sabendo-se que a soma e a multiplicação de elementos de $\overline{\mathbb{K}}_{f}$ são aplicações contínuas segue que

$$
S_{n} \cdot(1-x) \underset{n \rightarrow \infty}{\longrightarrow} z \cdot(1-x)
$$

Por outro lado, temos

$$
S_{n} \cdot(1-x)=\sum_{k=0}^{n} x^{k}-\sum_{k=1}^{n+1} x^{k}=1-x^{n+1} \underset{n \rightarrow \infty}{\longrightarrow} 1,
$$

pois $\left\|x^{n+1}\right\| \leq\|x\|^{n+1} \underset{n \rightarrow \infty}{\longrightarrow} 0$, o que implica $x^{n+1} \underset{n \rightarrow \infty}{\longrightarrow} 0$. Agora, Como $\left(\overline{\mathbb{K}}_{f}, \tau_{s f}\right)$ é Hausdorff segue que $z \cdot(1-x)=1$ e, portanto, $z \in \operatorname{Inv}\left(\overline{\mathbb{K}}_{f}\right)$ e $z=(1-x)^{-1}$. Logo,

$$
\sum_{n=0}^{\infty} x^{n}=z
$$

E segue o resultado.

Corolário 1.2.3. O conjunto dos invertiveis de $\overline{\mathbb{K}}_{f}$ que denotamos por $\operatorname{Inv}\left(\overline{\mathbb{K}}_{f}\right)$ é um subconjunto aberto de $\overline{\mathbb{K}}_{f}$.

Prova. Para mostrarmos isto, precisamos mostrar que para todo $a \in \operatorname{Inv}\left(\overline{\mathbb{K}}_{f}\right)$ existe $r>0$ tal que $B_{r}(a) \subset \operatorname{Inv}\left(\overline{\mathbb{K}}_{f}\right)$. Seja $a \in \operatorname{Inv}\left(\overline{\mathbb{K}}_{f}\right)$ e defina $r:=\left\|a^{-1}\right\|^{-1}$. Então se $z \in B_{r}(a)$, queremos mostrar que $z \in \operatorname{Inv}\left(\overline{\mathbb{K}}_{f}\right)$. De fato,

$$
\begin{aligned}
\left\|a^{-1}(z-a)\right\| & \leq\left\|a^{-1}\right\|\|z-a\| \\
& <r^{-1} r \\
& =1
\end{aligned}
$$


e, portanto, $\left\|a^{-1}(z-a)\right\|<1$ e pelo Proposição 1.2 .2 temos que $\left(1-a^{-1}(a-z)\right) \in \operatorname{Inv}\left(\overline{\mathbb{K}}_{f}\right)$. Agora, sendo $z=a\left(1-a^{-1}(a-z)\right)$ segue que $z \in \operatorname{Inv}\left(\overline{\mathbb{K}}_{f}\right)$, pois, por hipótese, $a \in \operatorname{Inv}\left(\overline{\mathbb{K}}_{f}\right)$. Em particular, $B_{r=1}(1) \subset \operatorname{Inv}\left(\overline{\mathbb{K}}_{f}\right)$.

Teorema 1.2.4. Seja $\mathfrak{I}$ um ideal próprio de $\overline{\mathbb{K}}_{f}$. Então para cada $x \in \mathfrak{I}$ temos $D(1, x) \geq 1$ e $D(1, \mathfrak{I})=1$. Portanto, todo ideal maximal de $\overline{\mathbb{K}}_{f}$ é fechado e assim, é também um conjunto raro.

Prova. Seja $\mathfrak{I}$ um ideal próprio de $\overline{\mathbb{K}}_{f}$. Então se $x \in \mathfrak{I}, x \notin \operatorname{Inv}\left(\overline{\mathbb{K}}_{f}\right)$ o que implica $D(1, x)=\|1-x\| \geq 1$, pois se $D(1, x)=\|1-x\|<1$, então pela Proposição 1.2.2, temos que $1-(1-x)=x \in \operatorname{Inv}\left(\overline{\mathbb{K}}_{f}\right)$ o que é um absurdo! Mais ainda, $D(1,0)=\|1\|=1$, i.e., $D(1,0)=1$. Isto mostra a primeira afirmação. Agora, se $\mathfrak{m}$ é um ideal maximal de $\overline{\mathbb{K}}_{f}$ segue da primeira parte que $1 \notin \overline{\mathrm{m}}$. E, portanto, temos a segunda afirmação.

Lema 1.2.5. (i) $0 \in \overline{\operatorname{Inv}\left(\overline{\mathbb{K}}_{f}\right)}$;

(ii) Para cada $x \in \overline{\mathbb{K}}_{f}$, se $r=-\mathrm{V}(x)$, então $y=\dot{\alpha}_{r} x \in S_{1}$;

(iii) Se $x \in \overline{\mathbb{K}}_{f}$, então $\mathrm{V}(x)+\mathrm{V}\left(x^{-1}\right) \leq 0$.

Prova. (i) Sabemos que $\left\{\dot{\alpha}_{n}\right\}_{n \in \mathbb{N}}$ é uma seqüência em $\operatorname{Inv}\left(\overline{\mathbb{K}}_{f}\right)$. Agora, pelo Corolário 1.1.12 (iv), fazendo $x=1$, temos

$$
\left\|\dot{\alpha}_{n}\right\|=e^{-n} \underset{n \rightarrow \infty}{\longrightarrow} 0
$$

E, portanto, $\dot{\alpha}_{n} \underset{n \rightarrow \infty}{\longrightarrow} 0$ o que mostrar que $0 \in \overline{\operatorname{Inv}\left(\overline{\mathbb{K}}_{f}\right)}$.

(ii) Para mostrarmos que $y \in S_{1}$, precisamos mostrar que $\|y\|=1$. De fato,

$$
\begin{aligned}
\|y\| & =\left\|\dot{\alpha}_{r} x\right\|(\text { pelo Corolário } 1.1 .12(i v), \text { temos }) \\
& =e^{-r}\|x\|(\text { como, por hipótese, }-r=\mathrm{V}(x), \text { temos }) \\
& =e^{\mathrm{V}(x)}\|x\| \\
& =\frac{1}{\|x\|}\|x\| \\
& =1
\end{aligned}
$$

portanto, $\|y\|=1$. 
(iii) Com efeito, $x \in \operatorname{Inv}\left(\overline{\mathbb{K}}_{f}\right)$, então $x \cdot x^{-1}=1$. Agora, pelo Corolário 1.1.12 (v), temos que

$$
\|1\|=1 \Rightarrow e^{-\mathrm{V}(1)}=1 \Rightarrow e^{-\mathrm{V}(1)}=e^{0} \Rightarrow \mathrm{V}(1)=0 .
$$

Assim,

$$
0=\mathrm{V}(1)=\mathrm{V}\left(x \cdot x^{-1}\right) \geq \mathrm{V}(x)+\mathrm{V}\left(x^{-1}\right)
$$

esta última desigualdade se deve a Proposição 1.1.10 (ii).

Proposição 1.2.6. (i) $\overline{\mathbb{K}}_{f}$ não tem ideais próprios aberto.

(ii) Nenhum módulo topológico $X$ sobre $\overline{\mathbb{K}}_{f}$ tem submódulos próprio aberto.

(iii) Se $X$ é um módulo topológico sobre $\overline{\mathbb{K}}_{f}$ Hausdorff, então para todo $x \in X, x \neq 0$ o conjunto $\operatorname{Inv}\left(\overline{\mathbb{K}}_{f}\right) \cdot x:=\left\{\lambda x \mid \lambda \in \operatorname{Inv}\left(\overline{\mathbb{K}}_{f}\right)\right\}$ não é um conjunto limitado e, portanto, $\operatorname{Inv}\left(\overline{\mathbb{K}}_{f}\right)$ não é um subconjunto limitado de $\overline{\mathbb{K}}_{f}$.

(iv) Um conjunto $B \subset \overline{\mathbb{K}}_{f}$ é limitado se, e somente se, é D-limitado.

(v) O único módulo topológico sobre $\overline{\mathbb{K}}_{f}$ que é limitado é (0) e, portanto, o único módulo topológico sobre $\overline{\mathbb{K}}_{f}$ que é compacto é o $(0)$.

Prova. (i) Com efeito, se $\mathfrak{I}$ é um ideal aberto de $\overline{\mathbb{K}}_{f}$, então pelo Lema 1.2.5 (i) temos que $\mathfrak{I} \cap \operatorname{Inv}\left(\overline{\mathbb{K}}_{f}\right) \neq \emptyset$ e, portanto, $\mathfrak{I}=\overline{\mathbb{K}}_{f}$ e $\mathfrak{I}$ não é um ideal próprio de $\overline{\mathbb{K}}_{f}$.

(ii) Suponhamos, por absurdo, que existe $X$ módulo topológico sobre $\overline{\mathbb{K}}_{f}$ tal que $S$ é submódulo próprio aberto de $X$. Então fixado $x_{0} \in X \backslash S$ temos pela continuidade da aplicação

$$
\lambda \in \overline{\mathbb{K}}_{f} \mapsto \lambda x_{0} \in X
$$

que o ideal $\mathfrak{I}:=\left\{\lambda \in \overline{\mathbb{K}}_{f} \mid \lambda x_{0} \in S\right\}$ é um ideal aberto próprio de $\overline{\mathbb{K}}_{f}$ o que é um absurdo pelo item (i). Logo, não existe tal módulo topológico $X$ sobre $\overline{\mathbb{K}}_{f}$.

(iii) Para mostrarmos que

$$
\operatorname{Inv}\left(\overline{\mathbb{K}}_{f}\right) \cdot x=\left\{\lambda x \mid \lambda \in \operatorname{Inv}\left(\overline{\mathbb{K}}_{f}\right)\right\}
$$

não é um conjunto limitado, precisamos mostrar que existe uma 0 -vizinhança $W$ de $X$ tal que para toda 0 -vizinhança $V$ de $\overline{\mathbb{K}}_{f}$ tem-se que $V \cdot\left(\operatorname{Inv}\left(\overline{\mathbb{K}}_{f}\right) \cdot x\right)$ não está contido em $W$. De fato, como $X$ é um módulo topológico sobre $\overline{\mathbb{K}}_{f}$ Hausdorff existe uma 0-vizinhança 
$W$ de $x$ tal que $x \notin W$. Vamos então mostrar que $x \in V$.(Inv $\left.\left(\overline{\mathbb{K}}_{f}\right) \cdot x\right)$ e, portanto, $W$ não contém $V \cdot\left(\operatorname{Inv}\left(\overline{\mathbb{K}}_{f}\right) \cdot x\right)$. De fato, pelo Lema $1.2 .5(i)$ temos que para toda 0 -vizinhança $V$ de $\overline{\mathbb{K}}_{f}$ existe $\lambda \in V \cap \operatorname{Inv}\left(\overline{\mathbb{K}}_{f}\right)$ e, portanto, $x=\lambda\left(\lambda^{-1} x\right) \in V$.(Inv $\left.\left(\overline{\mathbb{K}}_{f}\right) \cdot x\right)$ como queríamos.

(iv) Fixemos $r>0$ qualquer. Como $B_{t} B_{s} \subset B_{t s}, \forall t>0, s>0$ é natural que para todo $s>0$ existe $t>0$ tal que $B_{t} B_{r} \subset B_{s}$ e, portanto, todo conjunto $D$-limitado é limitado. Reciprocamente, se $L \subset \overline{\mathbb{K}}_{f}$ é limitado, existe $r>0$ tal que $B_{r} L \subset B_{1}$. Assim, pelo Lema 1.2.5 $(i)$ existe $\lambda \in B_{r} \cap \operatorname{Inv}\left(\overline{\mathbb{K}}_{f}\right) L \subset \lambda^{-1} B_{1}$. Agora, como existe $t \geq 1$ tal que $\lambda^{-1} B_{1} \subset B_{t}$ segue que $L \subset B_{t}$ e $L$ é $D$-limitado.

(v) A primeira afirmação segue imediatamente de (iii) e a segunda afirmação segue da primeira levando em conta que em um módulo topológico todo conjunto compacto é limitado.

Teorema 1.2.7. Se $\mathfrak{m}$ é um ideal maximal de $\overline{\mathbb{K}}_{f}$, então $\mathbb{K}$ pode ser identificado com um subcorpo $k$ de $L=\overline{\mathbb{K}}_{f} / \mathfrak{m}$ e $[L: \mathbb{K}]>1$, isto é, $L$ é uma extensão própria de $\overline{\mathbb{K}}_{f}$.

Prova. Seja $\pi: \overline{\mathbb{K}}_{f} \rightarrow L:=\overline{\mathbb{K}}_{f} / \mathfrak{m}$ a aplicação canônica. Então afirmamos que $k:=$ $\pi(\mathbb{K}) \simeq \mathbb{K}$. De fato, se $L=k$, então $\overline{\mathbb{K}}_{f}=\mathbb{K}+\mathfrak{m}$. Mas $\mathbb{K}$ é um subconjunto discreto de $\overline{\mathbb{K}}_{f}$ e, portanto, do Teorema 1.2.4 e a Proposição 1.2.6 (i) segue imediatamente que $\mathfrak{m} \cup \mathbb{K}$ é um conjunto fechado com interior vazio. Daí, existe $x \in B_{1}$, tal que $x \notin \mathfrak{m} \cup \mathbb{K}$. Então escrevamos $x=k_{x}-m_{x}$, onde $k_{x} \in \mathbb{K}$ e $m_{x} \in \mathfrak{m}$, assim $k_{x} \neq 0$ e, portanto, $m_{x}=k_{x}-x=k_{x}\left(1-k_{x}^{-1} x\right)$. De fato, $\left(1-k_{x}^{-1} x\right)=k_{x}^{-1} m_{x} \in \mathfrak{m}$, pois $\mathfrak{m}$ é um ideal, assim, $\left(1-k_{x}^{-1} x\right) \in \mathfrak{m}$ o que é um absurdo, pois $\left\|k_{x}^{-1} x\right\|=\|x\|<1$, pois $x \in B_{1}$ e, pela Proposição 1.2.2 segue que $1-k_{x}^{-1} x \in \operatorname{Inv}\left(\overline{\mathbb{K}}_{f}\right)$.

Lema 1.2.8. Sejam $R_{1}, R_{2}$ números reais positivos e defina $r:=\ln R_{1}-\ln R_{2}$. Então $\dot{\alpha}_{r} . S_{R_{1}}=S_{R_{2}}$. Em particular, se $x \in \overline{\mathbb{K}}_{f} \backslash\{0\}$ e $r=-\mathrm{V}(x)$, então $\dot{\alpha}_{r} x \in S_{1}$.

Prova. É conseqüência imediata do Corolário 1.1.12 (iv).

Teorema 1.2.9. $\overline{\mathbb{K}}_{f}$ não tem elementos nilpotentes e, portanto, seu nil-radical é trivial. Em particular, $\overline{\mathbb{K}}_{f}$ está contido num produto de domínios integrais.

Prova. Pelo Lema 1.2.8 é suficiente mostrarmos que $S_{1}$, a esfera de centro 0 e raio 1 , não tem elementos nilpotentes. Mas isto é claro, pois se existisse um tal elemento poderíamos facilmente mostrar que é nulo. Portanto, se $\mathfrak{P}$ denota o conjunto de todos os idéias primos de $\overline{\mathbb{K}}_{f}$, temos $\bigcap_{\mathfrak{p} \in \mathfrak{P}} \mathfrak{p}=0$, que é o kernel da aplicação natural $\overline{\mathbb{K}}_{f} \rightarrow \Pi_{\mathfrak{p} \in \mathfrak{P}} \overline{\mathbb{K}}_{f} / \mathfrak{p}$. 


\subsection{Tipos especiais de funções características}

Nosso objetivo nesta seção é estudar o grupo das unidades do anel dos números generalizados plenos de Colombeau, $\overline{\mathbb{K}}_{f}$, bem como os seus ideais primos e maximais. Para isto faremos primeiro uma análise cuidadosa do conjunto de zeros dos representantes dos elementos de $\overline{\mathbb{K}}_{f}$. E em seguida estudamos tipos especiais de funções características mostrando que elas estão relacionadas com os idéias primos e maximais de $\overline{\mathbb{K}}_{f}$. A nossa inspiração para esta pesquisa surgiu do artigo de Aragona e Juriaans [5].

Vamos agora definir o análogo ao conjunto $\mathcal{S}$ definido em [5] para o anel dos números generalizados plenos de Colombeau $\overline{\mathbb{K}}_{f}$ e o conjunto $P_{*}\left(\mathcal{S}_{f}\right)$. No restante deste trabalho usaremos com freqüência os símbolos $I, \bar{I}, I_{\eta}(\eta \in I), \mathcal{A}_{0}(\mathbb{K}), \mathcal{A}_{q}(\mathbb{K})(q \in \mathbb{N})$. Para $A \subset$ $\mathcal{A}_{0}(\mathbb{K})$ denote por $A^{c}$ o complementar de $A$ com respeito a $\mathcal{A}_{0}(\mathbb{K})$, i.e., o conjunto de todos os elementos $\varphi \in \mathcal{A}_{0}(\mathbb{K})$ tal que $\varphi \notin A$ e por $\hat{\mathcal{X}}_{A}$ a função característica de $A$ com domínio em $\mathcal{A}_{0}(\mathbb{K})$, i.e.,

$$
\hat{\mathcal{X}}_{A}(\varphi)= \begin{cases}1, & \text { se } \varphi \in A \\ 0, & \text { se } \varphi \in A^{c}\end{cases}
$$

É fácil ver que $\hat{\mathcal{X}}_{A} \in \mathcal{E}_{f}^{M}(\mathbb{K})$, mas $\hat{\mathcal{X}}_{A} \notin \mathcal{N}_{f}(\mathbb{K})$ e, portanto, $\hat{\mathcal{X}}_{A} \neq 0$. Denotemos por $\hat{\mathcal{X}}_{A}$ o representante de $\mathcal{X}_{A}$ em $\overline{\mathbb{K}}_{f}$. Isto, por abuso de linguagem, ainda será chamada de função característica de $A$.

Definição 1.3.1. Definimos

$$
\mathcal{S}_{f}:=\left\{A \subset \mathcal{A}_{0}(\mathbb{K}) \mid \forall p \in \mathbb{N}, \exists \varphi \in \mathcal{A}_{p}(\mathbb{K}) \text { tal que }\left\{\varepsilon \mid \varphi_{\varepsilon} \in A\right\} \in \mathcal{S}\right\},
$$

onde $\mathcal{S}:=\left\{S \subset I \mid 0 \in \bar{S} \cap \overline{S^{c}}\right\}$.

A proposição a seguir nos fornece duas propriedades importantes do conjunto $\mathcal{S}_{f}$ definido acima.

Proposição 1.3.2. (i) $A \in \mathcal{S}_{f} \Leftrightarrow A^{c} \in \mathcal{S}_{f}$.

(ii) $A \in \mathcal{S}_{f} \Rightarrow \emptyset \neq A \cap \mathcal{A}_{p}(\mathbb{K}) \varsubsetneqq \mathcal{A}_{p}(\mathbb{K}), \forall p \in \mathbb{N}$. Em particular, temos que $A \cap \mathcal{A}_{p}(\mathbb{K}) \neq$ $\emptyset$ e $A^{c} \cap \mathcal{A}_{p}(\mathbb{K}) \neq \emptyset$ para todo $p \in \mathbb{N}$.

Prova. (i) Com efeito, se $A \in \mathcal{S}_{f}$, então para todo $p \in \mathbb{N}$ existe $\varphi \in \mathcal{A}_{p}(\mathbb{K})$ tal que o $\left\{\varepsilon \mid \varphi_{\varepsilon} \in A\right\} \in \mathcal{S}$. Assim, pela (Proposição 4.2 item b pg. 2217 de Aragona e Juriaans [5]) 
temos que o $\left\{\varepsilon \mid \varphi_{\varepsilon} \in A\right\}^{c} \in \mathcal{S}$, mas $\left\{\varepsilon \mid \varphi_{\varepsilon} \in A\right\}^{c}=\left\{\varepsilon \mid \varphi_{\varepsilon} \notin A\right\}=\left\{\varepsilon \mid \varphi_{\varepsilon} \in A^{c}\right\}$ e, portanto, o $\left\{\varepsilon \mid \varphi_{\varepsilon} \in A^{c}\right\} \in \mathcal{S}$. Logo, para todo $p \in \mathbb{N}$ existe $\varphi \in \mathcal{A}_{p}(\mathbb{K})$ tal que o $\left\{\varepsilon \mid \varphi_{\varepsilon} \in A^{c}\right\} \in \mathcal{S}$ e, portanto, $A^{c} \in \mathcal{S}_{f}$. Reciprocamente, se $A^{c} \in \mathcal{S}_{f}$, então $A=\left(A^{c}\right)^{c} \in \mathcal{S}_{f}$ e, portanto, $A \in \mathcal{S}_{f}$.

(ii) Com efeito, se $A \in \mathcal{S}_{f}$, então para todo $p \in \mathbb{N}$, existe $\varphi \in \mathcal{A}_{p}(\mathbb{K})$ tal que o conjunto $A_{\varepsilon}:=\left\{\varepsilon \mid \varphi_{\varepsilon} \in A\right\} \in \mathcal{S}$. Como $\left\{\varphi_{\varepsilon} \mid \varepsilon \in A_{\varepsilon}\right\} \subseteq \mathcal{A}_{p}(\mathbb{K})$ e $\left\{\varphi_{\varepsilon} \mid \varepsilon \in A_{\varepsilon}\right\} \subseteq A$, segue que $A \cap \mathcal{A}_{p}(\mathbb{K}) \neq \emptyset$. Falta mostrarmos que $A \cap \mathcal{A}_{p}(\mathbb{K}) \varsubsetneqq \mathcal{A}_{p}(\mathbb{K})$. Que está contido é óbvio. Agora que $A \cap \mathcal{A}_{p}(\mathbb{K}) \neq \mathcal{A}_{p}(\mathbb{K})$ segue do fato que $\left\{\varphi_{\varepsilon} \mid \varepsilon \in A_{\varepsilon}^{c}\right\} \subset \mathcal{A}_{p}(\mathbb{K})$ e $\left\{\varphi_{\varepsilon} \mid \varepsilon \in A_{\varepsilon}^{c}\right\} \subset A^{c}$ que por $(i)$ também está em $\mathcal{S}_{f}$. Isto implica que $A^{c} \cap \mathcal{A}_{p}(\mathbb{K}) \neq \emptyset$. E, portanto, $A \cap \mathcal{A}_{p}(\mathbb{K}) \varsubsetneqq \mathcal{A}_{p}(\mathbb{K})$.

Proposição 1.3.3. Seja $A \in \mathcal{S}_{f}$. Então $\operatorname{Ann}\left(\mathcal{X}_{A}\right)=\overline{\mathbb{K}}_{f} \mathcal{X}_{A^{c}} e$

$$
\overline{\mathbb{K}}_{f}=\operatorname{Ann}\left(\mathcal{X}_{A}\right) \oplus \operatorname{Ann}\left(\mathcal{X}_{A^{c}}\right) \text {. }
$$

Para cada ideal primo $\mathfrak{p}$ de $\overline{\mathbb{K}}_{f}$ temos que ou $\mathcal{X}_{A}$ ou $\mathcal{X}_{A^{c}}=1-\mathcal{X}_{A}$ está em $\mathfrak{p}$.

Prova. Para mostrarmos que $\operatorname{Ann}\left(\mathcal{X}_{A}\right)=\overline{\mathbb{K}}_{f} \mathcal{X}_{A^{c}}$ precisamos mostrar que

$$
\operatorname{Ann}\left(\mathcal{X}_{A}\right) \subseteq \overline{\mathbb{K}}_{f} \mathcal{X}_{A^{c}} \quad \text { e } \quad \overline{\mathbb{K}}_{f} \mathcal{X}_{A^{c}} \subseteq \operatorname{Ann}\left(\mathcal{X}_{A}\right)
$$

De fato, se $x \in \operatorname{Ann}\left(\mathcal{X}_{A}\right)$, então $x \mathcal{X}_{A}=0$ e como $1=\mathcal{X}_{A}+\mathcal{X}_{A^{c}}$ segue multiplicando ambos os membros por $x$ que $x=x \mathcal{X}_{A^{c}} \in \overline{\mathbb{K}}_{f} \mathcal{X}_{A^{c}}$ e, portanto, Ann $\left(\mathcal{X}_{A}\right) \subseteq \overline{\mathbb{K}}_{f} \mathcal{X}_{A^{c}}$. Agora, se $x \in \overline{\mathbb{K}}_{f} \mathcal{X}_{A^{c}}$, então $x=y \mathcal{X}_{A^{c}}$ para algum $y \in \overline{\mathbb{K}}_{f}$ e, portanto,

$$
x \mathcal{X}_{A}=\left(y \mathcal{X}_{A^{c}}\right) \mathcal{X}_{A}=y\left(\mathcal{X}_{A^{c}} \mathcal{X}_{A}\right)=0
$$

pois $\mathcal{X}_{A}$ e $\mathcal{X}_{A^{c}}$ são idempotentes ortogonais. Isto mostra que $x \in \operatorname{Ann}\left(\mathcal{X}_{A}\right)$. Portanto, $\overline{\mathbb{K}}_{f} \mathcal{X}_{A^{c}} \subseteq \operatorname{Ann}\left(\mathcal{X}_{A}\right)$. Das duas inclusões segue a primeira assertiva. Falta agora, mostrarmos que

$$
\overline{\mathbb{K}}_{f}=\operatorname{Ann}\left(\mathcal{X}_{A}\right) \oplus \operatorname{Ann}\left(\mathcal{X}_{A^{c}}\right)
$$

Novamente, temos que sendo $1=\mathcal{X}_{A}+\mathcal{X}_{A^{c}}$ segue que multiplicando ambos os membros por $x \in \overline{\mathbb{K}}_{f}$, vem

$$
x=x \mathcal{X}_{A}+x \mathcal{X}_{A^{c}}
$$

com $x \mathcal{X}_{A} \in \operatorname{Ann}\left(\mathcal{X}_{A^{c}}\right)$ e $x \mathcal{X}_{A^{c}} \in \operatorname{Ann}\left(\mathcal{X}_{A}\right)$, pois $\mathcal{X}_{A}$ e $\mathcal{X}_{A^{c}}$ são idempotentes ortogonais. Logo, $\overline{\mathbb{K}}_{f} \subseteq \operatorname{Ann}\left(\mathcal{X}_{A}\right)+\operatorname{Ann}\left(\mathcal{X}_{A^{c}}\right)$. Agora, se $x \in \operatorname{Ann}\left(\mathcal{X}_{A}\right) \cap \operatorname{Ann}\left(\mathcal{X}_{A^{c}}\right)$, então $x \in \operatorname{Ann}\left(\mathcal{X}_{A}\right)$ 
e $x \in \operatorname{Ann}\left(\mathcal{X}_{A^{c}}\right)$, portanto, $x \mathcal{X}_{A}=0$ e $x \mathcal{X}_{A^{c}}=0$. Assim, $0=x \mathcal{X}_{A}+x \mathcal{X}_{A^{c}}=x \Rightarrow x=0$ o que completa a prova. Por último, como $\mathfrak{p} \ni 0=\mathcal{X}_{A} \mathcal{X}_{A^{c}}=\mathcal{X}_{A}\left(1-\mathcal{X}_{A^{c}}\right)$ segue que $\mathcal{X}_{A} \in \mathfrak{p}$ ou $\mathcal{X}_{A^{c}}=1-\mathcal{X}_{A} \in \mathfrak{p}$. Se ambos pertencem a $\mathfrak{p}$, então $1=\mathcal{X}_{A}+\mathcal{X}_{A^{c}} \in \mathfrak{p}$, absurdo.

Definição 1.3.4. Definimos, como é usual, $P\left(\mathcal{S}_{f}\right)$ como o conjunto das partes de $\mathcal{S}_{f}$, isto é, o conjunto de todos os subconjuntos de $\mathcal{S}_{f}$. Então denotamos por $P_{*}\left(\mathcal{S}_{f}\right)$ o conjunto de todos os subconjuntos $\mathcal{F}$ de $P\left(\mathcal{S}_{f}\right)$ satisfaz:

(i) Para.todo $A \in \mathcal{S}_{f}$, tem-se que $A$ ou $A^{c}$ está em $\mathcal{F}$, nunca ambos.

(ii) Se $A, B \in \mathcal{F}$, então $A \cup B \in \mathcal{F}$.

A segunda condição nos diz que $\mathcal{F}$ é estável por união finita. A proposição seguinte enumera algumas propriedades da função $\mathcal{X}_{A}$.

Proposição 1.3.5. (i) $S e A \in \mathcal{S}_{f}$, então $\mathcal{X}_{A} \in S_{1}$, i.e., $\left\|\mathcal{X}_{A}\right\|=1$.

(ii) $S e A, B \in \mathcal{F}, A \neq B$ e $\mathcal{X}_{A} \neq \mathcal{X}_{B}$, então $\mathrm{d}\left(\mathcal{X}_{A}, \mathcal{X}_{B}\right)=\left\|\mathcal{X}_{A}-\mathcal{X}_{B}\right\|=1$. Logo, $a$ topologia de $\overline{\mathbb{K}}_{f}$ não tem base enumerável. Isto mostra (ii) e (iii) da Proposição 1.1.14.

(iii) Se $A \in \mathcal{S}_{f}$, então $\mathcal{X}_{A} \in \overline{\mathbb{K}}_{f} \backslash\{0,1\}$ e $\mathcal{X}_{A}^{2}=\mathcal{X}_{A}$.

(iv) $\left(1-\mathcal{X}_{A}\right) \mathcal{X}_{A}=0$

Prova. (i) Para mostrarmos que $\left\|\mathcal{X}_{A}\right\|=1$ observemos que

$$
\dot{\alpha}_{-r} \approx 0 \Leftrightarrow r<0 .
$$

Assim, se $r \in \mathrm{A}\left(\mathcal{X}_{A}\right)$ (ver Definição 1.1.8), então $\dot{\alpha}_{-r} \mathcal{X}_{A} \approx 0$ e, portanto, $\dot{\alpha}_{-r} \approx 0$. E pela observação acima, temos que ter $r<0$, isto é, $\left.A\left(\mathcal{X}_{A}\right)=\right]-\infty, 0[$. Logo,

$$
\left\|\mathcal{X}_{A}\right\|=e^{-\mathrm{V}\left(\mathcal{X}_{A}\right)}=e^{-\sup \left(A\left(\mathcal{X}_{A}\right)\right)}=e^{0}=1 \text {. }
$$

(ii) Seja $r \in A\left(\mathcal{X}_{A}-\mathcal{X}_{B}\right)$. Então $\dot{\alpha}_{-r}\left(\mathcal{X}_{A}-\mathcal{X}_{B}\right) \approx 0$. Como $\left(\mathcal{X}_{A}-\mathcal{X}_{B}\right)$ não está associado a zero, pois $\mathcal{X}_{A} \neq \mathcal{X}_{B}$ segue que $\dot{\alpha}_{-r} \approx 0$. Novamente, pela observação acima devemos ter $r<0$ o que implica $\left.A\left(\mathcal{X}_{A}-\mathcal{X}_{B}\right)=\right]-\infty, 0[$. E, portanto,

$$
\left\|\mathcal{X}_{A}-\mathcal{X}_{B}\right\|=e^{-\mathrm{V}\left(\mathcal{X}_{A}-\mathcal{X}_{B}\right)}=e^{-\sup \left(A\left(\mathcal{X}_{A}-\mathcal{X}_{B}\right)\right)}=e^{0}=1 .
$$


(iii) Temos $\mathcal{X}_{A}^{2}=\mathcal{X}_{A} \cdot \mathcal{X}_{A}=\mathcal{X}_{A \cap A}=\mathcal{X}_{A}$. Portanto, $\mathcal{X}_{A}^{2}=\mathcal{X}_{A}$, i.e., $\mathcal{X}_{A}$ é um idempotente de $\overline{\mathbb{K}}_{f}$

(iv) Temos $\left(1-\mathcal{X}_{A}\right) \cdot \mathcal{X}_{A}=\mathcal{X}_{A}-\mathcal{X}_{A}^{2}=0$, pois por $(i i i)$ temos $\mathcal{X}_{A}^{2}=\mathcal{X}_{A}$.

Definição 1.3.6. Se $\mathcal{F} \in P_{*}\left(\mathcal{S}_{f}\right)$ definimos por

$$
g_{f}(\mathcal{F}):=\left\langle\left\{\mathcal{X}_{A} \mid A \in \mathcal{F}\right\}\right\rangle
$$

o ideal de $\overline{\mathbb{K}}_{f}$ gerado pelo conjunto de todas as funções características de A tal que $A \in \mathcal{F}$.

Lema 1.3.7. Se $\mathcal{F} \in P_{*}\left(\mathcal{S}_{f}\right)$ e $A, B \in \mathcal{F}$, então $\mathcal{X}_{A \cap B}, \mathcal{X}_{A \cup B} \in g_{f}(\mathcal{F})$.

Prova. Com efeito, se $A, B \in \mathcal{F}$, então pela Definição 1.3.4 (ii), temos que $A \cup B \in \mathcal{F}$. Agora, da Definição 1.3.6 temos que $\mathcal{X}_{A}, \mathcal{X}_{B}, \mathcal{X}_{A U B} \in g_{f}(\mathcal{F})$. Obviamente,

$$
\mathcal{X}_{A \cap B}=\mathcal{X}_{A} \mathcal{X}_{B} \in g_{f}(\mathcal{F})
$$

e, portanto, $\mathcal{X}_{A \cap B} \in g_{f}(\mathcal{F})$.

O lema a seguir garante que $g_{f}(\mathcal{F})$ é um ideal próprio de $\overline{\mathbb{K}}_{f}$. Mais precisamente, temos o seguinte:

Lema 1.3.8. Se $\mathcal{F} \in P_{*}\left(\mathcal{S}_{f}\right)$, então $g_{f}(\mathcal{F})$ é um ideal próprio de $\overline{\mathbb{K}}_{f}$, i.e., $g_{f}(\mathcal{F}) \nsupseteq \overline{\mathbb{K}}_{f}$.

Prova. Suponhamos que $g_{f}(\mathcal{F})=\overline{\mathbb{K}}_{f}$. Então existem $a_{i} \in \overline{\mathbb{K}}_{f}$ e $A_{i} \in \mathcal{F}, i=1,2, \ldots, n$ tal que

$$
1=\sum_{i=1}^{n} a_{i} \mathcal{X}_{A_{i}}
$$

Defina $A:=\bigcup_{i=1}^{n} A_{i}$. Então como $\mathcal{F} \in P_{*}\left(\mathcal{S}_{f}\right)$ e $\mathcal{F}$ é estável por união finita segue que $A \in \mathcal{F}$ e $A \neq \mathcal{A}_{0}(\mathbb{K})$, mais ainda, para todo $i=1,2, \ldots, n$ tem-se que $A_{i} \subset A$. Daí,

$$
\mathcal{X}_{A_{i}} \cdot \mathcal{X}_{A}=\mathcal{X}_{A_{i} \cap A}=\mathcal{X}_{A_{i}}
$$

Agora, multiplicando-se ambos os membros da equação (1.4) por $\mathcal{X}_{A}$, temos

$$
\mathcal{X}_{A}=\sum_{i=1}^{n} a_{i} \mathcal{X}_{A_{i}} \cdot \mathcal{X}_{A}=\sum_{i=1}^{n} a_{i} \mathcal{X}_{A_{i}}=1
$$

Logo,

$$
\mathcal{X}_{A}=1 \Rightarrow \mathcal{X}_{A}-1=0 \Rightarrow\left(\hat{\mathcal{X}}_{A}-1\right) \in \mathcal{N}_{f}(\mathbb{K})
$$


Pela Definição 1.1.5 que define as "nulas" de $\overline{\mathbb{K}}_{f}$, temos que $\exists p \in \mathbb{N}, \exists \gamma \in \Gamma$ tal que $\forall q \geq p$ e $\forall \varphi \in \mathcal{A}_{q}(\mathbb{K})\left(\exists C=C_{\varphi}>0, \exists \eta=\eta_{\varphi}>0\right)$ tal que

$$
\left|1-\hat{\mathcal{X}}_{A}\left(\varphi_{\varepsilon}\right)\right| \leq C \varepsilon^{\gamma(q)-p}, \forall 0<\varepsilon<\eta
$$

onde a $\hat{\mathcal{X}}_{A}$ é um representante da $\mathcal{X}_{A}$ em $\overline{\mathbb{K}}_{f}$. Mas $A \in \mathcal{F} \subset P\left(\mathcal{S}_{f}\right)$. Logo, para este $\varphi$ temos que o

$$
\left\{\varepsilon \mid \varphi_{\varepsilon} \in A\right\} \in \mathcal{S} \Rightarrow 0 \in \overline{\left\{\varepsilon \mid \varphi_{\varepsilon} \in A\right\}} \cap \overline{\left\{\varepsilon \mid \varphi_{\varepsilon} \in A^{c}\right\}}
$$

isto é, existe uma seqüência $\left\{\varepsilon_{n}\right\}_{n \in \mathbb{N}}$ que converge a zero quando $n \rightarrow \infty$ tal que $\varphi_{\varepsilon_{n}} \in A^{c}$. Substituindo isto em (1.5), obtemos

$$
1=\left|1-\hat{\mathcal{X}}_{A}\left(\varphi_{\varepsilon_{n}}\right)\right| \leq C\left(\varepsilon_{n}\right)^{\gamma(q)-p}
$$

Como $\gamma$ é divergente, podemos escolher $q_{0} \in \mathbb{N}$ tal que $\gamma\left(q_{0}\right)-p>0$. Assim, temos $1 \leq C\left(\varepsilon_{n}\right)^{\gamma\left(q_{0}\right)-p} \underset{n \longrightarrow \infty}{\longrightarrow} 0$ o que é um absurdo. Portanto, $g_{f}(\mathcal{F}) \pitchfork \overline{\mathbb{K}}_{f}$.

Teorema 1.3.9. Para todo ideal primo $\mathfrak{p}$ de $\overline{\mathbb{K}}_{f}$ existe um único $\mathcal{F}_{\mathfrak{p}} \in P_{*}\left(\mathcal{S}_{f}\right)$ tal que $g_{f}\left(\mathcal{F}_{\mathrm{p}}\right) \subset \mathfrak{p}$. Em particular, $P_{*}\left(\mathcal{S}_{f}\right) \neq \emptyset$.

Prova. Seja $\mathfrak{p}$ um ideal primo de $\overline{\mathbb{K}}_{f}$. Então $\mathfrak{p}$ é um ideal próprio e como $\mathcal{X}_{A}+\mathcal{X}_{A^{c}}=1$ segue da Proposição 1.3.3 que $\mathcal{F}_{\mathfrak{p}}=\left\{A \in \mathcal{S}_{f} \mid \mathcal{X}_{A} \in \mathfrak{p}\right\}$ satisfaz a condição $(i)$ da Definição 1.3.4. Agora, dados $A, B \in \mathcal{F}_{\mathfrak{p}}$, queremos mostrar que $A \cup B \in \mathcal{F}_{\mathfrak{p}}$. Isto é, $\mathcal{F}_{\mathbf{p}}$ satisfaz a condição (ii) da Definição 1.3.4. De fato, se $A, B \in \mathcal{F}_{\mathfrak{p}}$, então $\mathcal{X}_{A}, \mathcal{X}_{B} \in \mathfrak{p}$ e sendo

$$
\mathcal{X}_{A \cap B}=\mathcal{X}_{A} \mathcal{X}_{B}
$$

segue que $\mathcal{X}_{A \cap B} \in \mathfrak{p}$, pois $\mathcal{X}_{A} \mathcal{X}_{B} \in \mathfrak{p}$. Logo,

$$
\mathcal{X}_{A \cup B}=\mathcal{X}_{A}+\mathcal{X}_{B}-\mathcal{X}_{A \cap B} \in \mathfrak{p}
$$

Assim, é suficiente mostrarmos que $A \cup B \in \mathcal{S}_{f}$. Suponhamos, por absurdo, que $A \cup B \notin \mathcal{S}_{f}$. Então $\mathcal{X}_{A \cup B}=0$ ou $\mathcal{X}_{A \cup B}=1$. Se $\mathcal{X}_{A \cup B}=0$, então por (1.6) segue que

$$
\mathcal{X}_{A}+\mathcal{X}_{B}=\mathcal{X}_{A \cap B}
$$

Agora, multiplicando ambos os membros de (1.7) por $\mathcal{X}_{A}$, vem

$$
\mathcal{X}_{A}\left(\mathcal{X}_{A}+\mathcal{X}_{B}\right)=\mathcal{X}_{A \cap B}
$$


como

$$
\mathcal{X}_{A}+\mathcal{X}_{A \cap B}=\mathcal{X}_{A}^{2}+\mathcal{X}_{A} \mathcal{X}_{B}=\mathcal{X}_{A}\left(\mathcal{X}_{A}+\mathcal{X}_{B}\right)
$$

segue por (1.8) que

$$
\mathcal{X}_{A}+\mathcal{X}_{A \cap B}=\mathcal{X}_{A \cap B}
$$

e, portanto, $\mathcal{X}_{A}=0$ o que implica $A \notin \mathcal{S}_{f}$, absurdo. Agora, se $\mathcal{X}_{A \cup B}=1$, novamente por (1.6) temos que $1 \in \mathfrak{p}$ o que é um absurdo, pois $\mathfrak{p}$ é um ideal próprio de $\overline{\mathbb{K}}_{f}$. Logo, $A \cup B \in \mathcal{S}_{f}$. Das condições $(i)$ e (ii) da Definição 1.3.4 satisfeitas concluímos que

$$
\mathcal{F}_{\mathrm{p}} \in P_{*}\left(\mathcal{S}_{f}\right)
$$

Para mostrarmos que $g_{f}\left(\mathcal{F}_{\mathfrak{p}}\right) \subset \mathfrak{p}$ seja $\mathcal{X}_{A} \in g_{f}\left(\mathcal{F}_{\mathfrak{p}}\right)$. Então pela Definição 1.3.6 $A \in \mathcal{F}_{\mathfrak{p}}$ e, portanto, $\mathcal{X}_{A} \in \mathfrak{p}$ o que implica $g_{f}\left(\mathcal{F}_{\mathfrak{p}}\right) \subset \mathfrak{p}$. A unicidade de $\mathcal{F}_{\mathfrak{p}}$ segue do fato que $\mathcal{X}_{A}$ e $\mathcal{X}_{A^{c}}$ são idempotentes ortogonais cuja a soma é igual a 1 e que p é um ideal próprio de $\overline{\mathbb{K}}_{f}$. Por último mostramos que $P_{*}\left(\mathcal{S}_{f}\right) \neq \emptyset$, pois para cada ideal primo $\mathfrak{p}$ de $\overline{\mathbb{K}}_{f}$ temos que $\mathcal{F}_{\mathrm{p}} \in P_{*}\left(\mathcal{S}_{f}\right)$.

O Teorema 1.3.9 associa a cada ideal primo $\mathfrak{p}$ de $\overline{\mathbb{K}}_{f}$ um conjunto bem definido

$$
\mathcal{F}_{\mathfrak{p}} \in P_{*}\left(\mathcal{S}_{f}\right)
$$

caracterizado pela inclusão $g_{f}\left(\mathcal{F}_{\mathfrak{p}}\right) \subset \mathfrak{p}$.

Definição 1.3.10. Se $x \in \overline{\mathbb{K}}_{f}$ e $\hat{x}$ é um representante de $x$ definimos

$$
Z(\hat{x}):=\left\{\varphi \in \mathcal{A}_{0}(\mathbb{K}) \mid \hat{x}(\varphi)=0\right\}
$$

o conjunto de zeros do representante $\hat{x}$ de $x \in \overline{\mathbb{K}}_{f}$.

Lema 1.3.11. Seja $x \in \overline{\mathbb{K}}_{f} \backslash\{0\}$ e $\mathcal{F} \in P_{*}\left(\mathcal{S}_{f}\right)$. Então as seguintes afirmações são equivalentes:

(i) $x \in g_{f}(\mathcal{F})$.

(ii) Existe $A \in \mathcal{F}$ tal que $x \mathcal{X}_{A}=x$.

Prova. $(i) \Rightarrow(i i)$ : Com efeito, se $x \in g_{f}(\mathcal{F})$, então existem $A_{1}, A_{2}, \ldots, A_{n} \in \mathcal{F}$ e $a_{1}, a_{2}, \ldots, a_{n} \in \overline{\mathbb{K}}_{f}$ tal que

$$
x=\sum_{i=1}^{n} a_{i} \mathcal{X}_{A_{i}} .
$$


Pela Definição 1.3.4 (ii) temos que

$$
A:=\bigcup_{i=1}^{n} A_{i} \in \mathcal{F}
$$

e como $A_{i} \subset A, \forall i=1,2, \ldots, n$ segue que $\mathcal{X}_{A_{i}} \mathcal{X}_{A}=\mathcal{X}_{A_{i} \cap A}=\mathcal{X}_{A_{i}}$. Logo,

$$
\begin{aligned}
x \mathcal{X}_{A} & =\left(\sum_{i=1}^{n} a_{i} \mathcal{X}_{A_{i}}\right) \mathcal{X}_{A} \\
& =\sum_{i=1}^{n}\left(a_{i} \mathcal{X}_{A_{i}}\right) \mathcal{X}_{A} \\
& =\sum_{i=1}^{n} a_{i}\left(\mathcal{X}_{A_{i}} \mathcal{X}_{A}\right) \\
& =\sum_{i=1}^{n} a_{i} \mathcal{X}_{A_{i}}=x
\end{aligned}
$$

Portanto, $x \mathcal{X}_{A}=x$ e $A \in \mathcal{F}$.

(ii) $\Rightarrow(i)$ : Com efeito, se existe $A \in \mathcal{F}$ tal que $x \mathcal{X}_{A}=x$, então $x \in g_{f}(\mathcal{F})$, pois existe $A \in \mathcal{F}$ e $x \in \overline{\mathbb{K}}_{f}$ tal que $x=x \mathcal{X}_{A}$, onde $\mathcal{X}_{A} \in g_{f}(\mathcal{F})$

Seguinte teorema relaciona os elementos invertíveis de $\overline{\mathbb{K}}_{f}$ com o conjunto de zeros de seus representantes, mais precisamente, temos o seguinte:

Teorema 1.3.12. $\quad(i) x \in \operatorname{Inv}\left(\overline{\mathbb{K}}_{f}\right) \Leftrightarrow Z(\hat{x}) \notin \mathcal{S}_{f}, \forall$ representante $\hat{x}$ de $x$.

(ii) $x \notin \operatorname{Inv}\left(\overline{\mathbb{K}}_{f}\right) \Leftrightarrow \exists e \in \overline{\mathbb{K}}_{f}, e^{2}=e$ (idempotente) tal que x.e $=0$. Em particular, se $x \in \overline{\mathbb{K}}_{f} \backslash\{0\}$ e $x \notin \operatorname{Inv}\left(\overline{\mathbb{K}}_{f}\right)$, então $x$ é um divisor de zero.

Prova. (i) Suponhamos, por absurdo, que $x \in \operatorname{Inv}\left(\overline{\mathbb{K}}_{f}\right)$ e que $Z(\hat{x}) \in \mathcal{S}_{f}$ para algum representante $\hat{x}$ de $x$. Então, pela Proposição 1.3 .5 (iii), temos que $\hat{\mathcal{X}}_{Z(\hat{x})} \notin\{0,1\}$, assim $\hat{\mathcal{X}}_{Z(\hat{x})} \neq 0$, mas $x \mathcal{X}_{Z(\hat{x})}=0$ e, portanto, $x$ é um divisor de zero, absurdo. Logo, $Z(\hat{x}) \notin$ $\mathcal{S}_{f}$, qualquer que seja o representante $\hat{x}$ de $x$. Para mostrarmos a recíproca é suficiente mostrarmos (ii).

(ii) Vamos agora mostrar que se $x \in \overline{\mathbb{K}}_{f} \backslash\{0\}$ e $x \notin \operatorname{Inv}\left(\overline{\mathbb{K}}_{f}\right)$, então $x$ é um divisor de zero. Seja $\hat{x}$ um representante de $x$. Então, temos dois casos a estudar, a saber:

(a) $Z(\hat{x}) \in \mathcal{S}_{f}$. 
(b) $Z(\hat{x}) \notin \mathcal{S}_{f}$.

(a) Se $Z(\hat{x}) \in \mathcal{S}_{f}$, então da Proposição 1.3.5 (iii), temos que $\hat{\mathcal{X}}_{Z(\hat{x})} \neq 0$, mas $x \mathcal{X}_{Z(\hat{x})}=0$ o que implica que $x$ é um divisor de zero.

(b) Agora, se $Z(\hat{x}) \notin \mathcal{S}_{f}$. Defina

$$
x^{*}(\varphi)= \begin{cases}\hat{x}(\varphi), & \text { se } \varphi \notin Z(\hat{x}) \\ 0, & \text { se } \varphi \in Z(\hat{x})\end{cases}
$$

então $x^{*}(\varphi) \neq 0$ para alguma $\varphi \in \mathcal{A}_{0}(\mathbb{K})$. Claramente, temos que $\left(x^{*}-\hat{x}\right) \in \mathcal{N}_{f}(\mathbb{K})$. Assim, podemos substituir $\hat{x}$ por $x^{*}$, ou melhor, supor que $\hat{x}(\varphi) \neq 0$. Como, por hipótese, $x \notin \operatorname{Inv}\left(\overline{\mathbb{K}}_{f}\right)$ segue que $\frac{1}{x} \notin \mathcal{E}_{f}^{M}(\mathbb{K})$, pois se $\frac{1}{x} \in \mathcal{E}_{f}^{M}(\mathbb{K})$, então sendo $\frac{1}{\hat{x}}$ seu representante, vem que $\hat{x} \frac{1}{\hat{x}}=1 \Rightarrow x \operatorname{cl}\left[\frac{1}{\hat{x}}\right]=x \frac{1}{x}=1 \Rightarrow x \in \operatorname{Inv}\left(\overline{\mathbb{K}}_{f}\right)$, absurdo! Mas $\frac{1}{x} \notin \mathcal{E}_{f}^{M}(\mathbb{K})$ implica pela Definição 1.1 .5 das moderada que $\forall p \in \mathbb{N}, \exists \varphi \in \mathcal{A}_{p}(\mathbb{K})$ tal que $\forall C=C_{\varphi}>$ $0, \forall \eta=\eta_{\varphi}>0, \exists 0<\varepsilon_{0}<\eta$ tal que $\left|\frac{1}{\hat{x}}\left(\varphi_{\varepsilon_{0}}\right)\right|>C \varepsilon_{0}^{-p}$. Tomemos $C=n, \eta=\frac{1}{n}$, temos que existe $0<\varepsilon_{n}<\frac{1}{n}$ tal que

$$
\left|\frac{1}{\hat{x}}\left(\varphi_{\varepsilon_{n}}\right)\right|>n \varepsilon_{n}^{-p} .
$$

Agora, para esta $\varphi$ defina o conjunto $A_{p}\left(\varphi_{p}\right):=\left\{\varepsilon_{n} \mid n \in \mathbb{N}\right\}$. Seja

$$
B:=\left\{\left(\varphi_{p}\right)_{\varepsilon_{n}} \mid \varepsilon_{n} \in A_{p}\left(\varphi_{p}\right)\right\}
$$

Então $B \in \mathcal{S}_{f}$, i.e., $\forall p \in \mathbb{N}$, exists $\varphi_{p} \in \mathcal{A}_{p}(\mathbb{K})$ tal que $\left\{\varepsilon_{n} \mid\left(\varphi_{p}\right)_{\varepsilon_{n}} \in B\right\} \in \mathcal{S}$. De fato, basta mostrarmos que $\left\{\varepsilon_{n} \mid\left(\varphi_{p}\right)_{\varepsilon_{n}} \in B\right\} \in \mathcal{S}$. Para isto é suficiente mostrarmos que

$$
A_{p}\left(\varphi_{p}\right)=\left\{\varepsilon_{n} \mid\left(\varphi_{p}\right)_{\varepsilon_{n}} \in B\right\}
$$

pois $\emptyset \neq A_{p}\left(\varphi_{p}\right) \cap I_{\eta=1 / 2} \neq I_{\eta=1 / 2}^{2}$ já que basta tomarmos $0<\varepsilon_{n}<1 / 2$. Isto é possível, pois

$$
\lim _{n \rightarrow \infty} \varepsilon_{n} \leq \lim _{n \rightarrow \infty} \frac{1}{n}=0
$$

e, portanto,

$$
\lim _{n \rightarrow \infty} \varepsilon_{n}=0 .
$$

Seja $a \in A_{p}\left(\varphi_{p}\right)$, então $a=\varepsilon_{n}$ para algum $n \in \mathbb{N}$ e pela definição de $B$, temos que

$$
\left(\varphi_{p}\right)_{a}=\left(\varphi_{p}\right)_{\varepsilon_{n}} \in B
$$

\footnotetext{
${ }^{2}$ Condição necessária e suficiente para que um conjunto pertença a $\mathcal{S}$.
} 
e, portanto, $a \in\left\{\varepsilon_{n} \mid\left(\varphi_{p}\right)_{\varepsilon_{n}} \in B\right\}$. Daí, temos que

$$
A_{p}\left(\varphi_{p}\right) \subseteq\left\{\varepsilon_{n} \mid\left(\varphi_{p}\right)_{\varepsilon_{n}} \in B\right\}
$$

Reciprocamente, se $a \in\left\{\varepsilon_{n} \mid\left(\varphi_{p}\right)_{\varepsilon_{n}} \in B\right\}$, então $a=\varepsilon_{n}$ para algum $n \in \mathbb{N}$ e

$$
\left(\varphi_{p}\right)_{a}=\left(\varphi_{p}\right)_{\varepsilon_{n}} \in B
$$

e, novamente, pela definição de $B$, temos que $a \in A_{p}\left(\varphi_{p}\right)$ e, portanto,

$$
\left\{\varepsilon_{n} \mid\left(\varphi_{p}\right)_{\varepsilon_{n}} \in B\right\} \subseteq A_{p}\left(\varphi_{p}\right)
$$

Das duas inclusões temos que $A_{p}\left(\varphi_{p}\right)=\left\{\varepsilon_{n} \mid \varphi_{p, \varepsilon_{n}} \in B\right\}$. E, portanto, $\left\{\varepsilon_{n} \mid\left(\varphi_{p}\right)_{\varepsilon_{n}} \in B\right\} \in \mathcal{S}$ o que mostrar que $B \in \mathcal{S}_{f}$. Vamos, agora, mostrar que $\hat{x} \hat{\mathcal{X}}_{B} \in \mathcal{N}_{f}(\mathbb{K})$. Pela Definição 1.1.5 das nulas, precisamos mostrar que $\exists p \in \mathbb{N} \exists \gamma \in \Gamma$ tal que $\forall q \geq p, \forall \varphi \in \mathcal{A}_{q}(\mathbb{K}) \exists C=$ $C(\varphi)>0, \exists \eta=\eta(\varphi)>0$ tal que $\left|\hat{x} \hat{\mathcal{X}}_{B}\left(\varphi_{\varepsilon}\right)\right| \leq C \varepsilon^{\gamma(q)-p}, \forall 0<\varepsilon<\eta$. De fato, basta tomarmos $p=0, \gamma(q)=q$, pois $\left|\hat{x} \hat{\mathcal{X}}_{B}\left(\varphi_{\varepsilon}\right)\right| \leq C \varepsilon^{q}<1$ para todo $\varepsilon$ suficientemente pequeno. E, portanto, $\hat{x} \hat{\mathcal{X}}_{B} \in \mathcal{N}_{f}(\mathbb{K})$. Agora, observe que $\hat{z}(\varphi):=\left(\hat{x}-\hat{x} \hat{\mathcal{X}}_{B}\right)(\varphi)=$ $0, \forall \varphi \in B \Rightarrow B \subseteq Z(\hat{z}) \Rightarrow Z(\hat{z}) \in \mathcal{S}_{f}$, mais ainda, $\hat{z}$ é um representante de $x$, pois $\hat{x}-\hat{z}=\hat{x} \hat{\mathcal{X}}_{B} \in \mathcal{N}_{f}(\mathbb{K})$. Logo, $\hat{x} \hat{\mathcal{X}}_{Z(\hat{z})}=0$, com $\hat{\mathcal{X}}_{Z(\hat{z})} \neq 0$, o que mostra que $x$ é um divisor de zero.

Definição 1.3.13. Dado $x \in \mathcal{E}_{f}^{M}(\mathbb{K})$ e a $\in \mathbb{N}$ definimos

(i) $N_{a}(x):=\left\{\varphi \in \mathcal{A}_{0}(\mathbb{K}) \| x(\varphi) \mid<\dot{\alpha}_{a}(\varphi)\right\}$

(ii) $\hat{\mathcal{X}}_{a, x}:=\hat{\mathcal{X}}_{N_{a}(x)}$ e $\mathcal{X}_{a, x}:=\mathcal{X}_{N_{a}(x)}$

Lema 1.3.14. Seja $A \subseteq \mathcal{A}_{0}(\mathbb{K})$. Então $\left.\left.\hat{\mathcal{X}}_{A} \in \mathcal{N}_{f}(\mathbb{K}) \Leftrightarrow \exists \tau: \mathcal{A}_{0}(\mathbb{K}) \rightarrow\right] 0,1\right]$ tal que para todo $\varphi \in \mathcal{A}_{q}(\mathbb{K})$ tem-se que $I_{\tau(\varphi)} \subseteq\left\{\varepsilon \mid \varphi_{\varepsilon} \in A^{c}\right\}$.

Prova. Temos pela Definição 1.1.5 que $\hat{\mathcal{X}}_{A} \in \mathcal{N}_{f}(\mathbb{K}) \Leftrightarrow \exists p \in \mathbb{N}, \exists \gamma \in \Gamma$ tal que $\forall q \geq$ $p, \forall \varphi \in \mathcal{A}_{q}(\mathbb{K}) \exists C=C_{\varphi}>0, \exists \eta=\eta_{\varphi}>0$ tal que $\left|\hat{\mathcal{X}}_{A}\left(\varphi_{\varepsilon}\right)\right| \leq C \varepsilon^{\gamma(q)-p}, \forall 0<\varepsilon<\eta$. Como $\gamma$ é divergente, podemos escolher $q$ suficientemente grande de modo que $\gamma(q)-p>2$ em seguida tomamos $\varepsilon$ suficientemente pequeno, digamos $0<\varepsilon<\tau(\varphi)<\eta=\eta_{\varphi}$, de modo que $C \varepsilon^{\gamma(q)-p}<1$. Neste caso, temos que $\left|\hat{\mathcal{X}}_{A}\left(\varphi_{\varepsilon}\right)\right|<1 \Leftrightarrow \hat{\mathcal{X}}_{A}\left(\varphi_{\varepsilon}\right)=0 \Leftrightarrow \varphi_{\varepsilon} \in A^{c} \Leftrightarrow$ $\left\{\varphi_{\varepsilon} \mid \varepsilon<\tau(\varphi)\right\} \subseteq A^{c}$, i.e, $\left\{\varphi_{\varepsilon} \mid \varepsilon \in I_{\tau(\varphi)}\right\} \subseteq A^{c}$. Equivalentemente, $I_{\tau(\varphi)} \subseteq\left\{\varepsilon \mid \varphi_{\varepsilon} \in A^{c}\right\}$. 
Lema 1.3.15. (i) $\mathcal{X}_{N_{a}(x)}=1$ para todo $a \in \mathbb{N}$ se, e somente se, $x \in \mathcal{N}_{f}(\mathbb{K})$.

(ii) $\mathcal{X}_{N_{a}(x)}=0$ para todo $a \in \mathbb{N}$ se, e somente se, $x \in \operatorname{Inv}\left(\overline{\mathbb{K}}_{f}\right)$.

Prova. (i) Tomemos, então $p=0, \gamma: \mathbb{N} \rightarrow \mathbb{N}$ tal que $\gamma(q)=q \geq 0$. Temos, por hipótese, que $\mathcal{X}_{N_{q}(x)}=1$ se, e somente se, $\hat{\mathcal{X}}_{\left(N_{q}(x)\right)^{c}}=1-\hat{\mathcal{X}}_{N_{q}(x)} \in \mathcal{N}_{f}(\mathbb{K})$ e pelo Lema 1.3 .14 se, e somente se, existe $\left.\left.\tau: \mathcal{A}_{0}(\mathbb{K}) \rightarrow\right] 0,1\right]$ tal que para toda $\varphi \in \mathcal{A}_{q}(\mathbb{K})$ tem-se que $I_{\tau(\varphi)} \subseteq\left\{\varepsilon \mid \varphi_{\varepsilon} \in N_{q}(x)\right\}$. Pela Definição 1.3.13 de $N_{q}(x)$ se, e somente se,

$$
\left|x\left(\varphi_{\varepsilon}\right)\right|<\dot{\alpha}_{q}\left(\varphi_{\varepsilon}\right)=(i(\varphi))^{q} \varepsilon^{q}, \forall 0<\varepsilon<\tau(\varphi)
$$

se, e somente se, $x \in \mathcal{N}_{f}(\mathbb{K})$, pois dado $\varphi \in \mathcal{A}_{q}(\mathbb{K})$ tomemos $C=C_{\varphi}=(i(\varphi))^{q}>0, \eta=$ $\eta_{\varphi}=\tau(\varphi)>0$ e obtemos

$$
\left|x\left(\varphi_{\varepsilon}\right)\right|<(i(\varphi))^{q} \varepsilon^{q}=C \varepsilon^{\gamma(q)-p}, \forall 0<\varepsilon<\eta(\varphi)^{3}
$$

Para mostrarmos (ii) usamos um raciocínio análogo ao de $(i)$.

Proposição 1.3.16. Seja $x \in \overline{\mathbb{K}}_{f} \backslash\{0\}$ uma não-unidade. Então existe a $\in \mathbb{N}$ tal que $S=N_{a}(x) \in \mathcal{S}_{f}$ e $\left|x \mathcal{X}_{S}\right|<\dot{\alpha}_{a}$

Prova. Suponhamos, por absurdo, que para todo $a \in \mathbb{N}$ tem-se que

$$
S:=N_{a}(x) \notin \mathcal{S}_{f}
$$

Então $\mathcal{X}_{N_{a}(x)} \in \mathcal{N}_{f}(\mathbb{K})$ ou $\mathcal{X}_{\left(N_{a}(x)\right)^{c}} \in \mathcal{N}_{f}(\mathbb{K})$. De fato, se $\mathcal{X}_{N_{a}(x)} \in \mathcal{N}_{f}(\mathbb{K})$, então pelo Lema 1.3.14 existe

$$
\left.\left.\tau: \mathcal{A}_{0}(\mathbb{K}) \rightarrow\right] 0,1\right]
$$

tal que para toda $\varphi \in \mathcal{A}_{q}(\mathbb{K})$ tem-se que $I_{\tau(\varphi)} \subseteq\left\{\varepsilon \mid \varphi_{\varepsilon} \in\left(N_{a}(x)\right)^{c}\right\}$. Logo, pela Definição 1.3.13 de $N_{a}(x)$ temos que

$$
\left|x\left(\varphi_{\varepsilon}\right)\right| \geq \dot{\alpha}_{a}\left(\varphi_{\varepsilon}\right)=(i(\varphi))^{a} \varepsilon^{a}, \forall 0<\varepsilon<\tau(\varphi)
$$

e, portanto, $\left|\frac{1}{x\left(\varphi_{\varepsilon}\right)}\right| \leq(i(\varphi))^{-a} \varepsilon^{-a}, \forall 0<\varepsilon<\tau(\varphi)$ o que implica que $\frac{1}{x} \in \mathcal{E}_{f}^{M}(\mathbb{K})$ e sendo $x \frac{1}{x}=1$ segue que $x \in \operatorname{Inv}\left(\overline{\mathbb{K}}_{f}\right)$ o que é um absurdo, pois, por hipótese, $x$ não é uma unidade. Agora, se $\mathcal{X}_{\left(N_{a}(x)\right)^{c}} \in \mathcal{N}_{f}(\mathbb{K})$, então sendo $\mathcal{X}_{\left(N_{a}(x)\right)^{c}}=1-\mathcal{X}_{N_{a}(x)}$ segue que

\footnotetext{
${ }^{3}$ Veja a Definição 1.1 .5 das nulas.
} 
$\mathcal{X}_{N_{a}(x)}=1, \forall a \in \mathbb{N}$ e pelo Lema 1.3.15 (i) segue-se que $x \in \mathcal{N}_{f}(\mathbb{K})$ o que é um absurdo, pois, por hipótese, $x \neq 0$. Das duas hipóteses verificadas segue então que existe $a \in \mathbb{N}$ tal que $S=N_{a}(x) \in \mathcal{S}_{f}$. A última afirmação segue imediatamente da definição de $S$.

Vamos agora, enunciar e demonstrar o Teorema da Aproximação para o anel dos números generalizados plenos de colombeau $\overline{\mathbb{K}}_{f}$. Este teorema será uma ferramenta fundamental para provar o resultado principal deste capítulo.

Teorema 1.3.17 (Teorema da aproximação). Seja $x \in \overline{\mathbb{K}}_{f} \backslash\{0\}$ uma não-unidade. Então $x$ satisfaz uma e somente uma das condições abaixo:

(a) Existe $S \in \mathcal{S}_{f}$ e $a \in \mathbb{N}$ tal que

(i) $x \mathcal{X}_{S}=0$;

(ii) $\left|x \mathcal{X}_{S^{c}}\right| \geq \dot{\alpha}_{a} \mathcal{X}_{S^{c}}$ (i.e., existe $\hat{x}$ representante de $x$ tal que

$$
\left.|\hat{x}(\varphi)| \geq \hat{\dot{\alpha}}_{a}(\varphi), \forall \varphi \in \mathcal{A}_{0}(\mathbb{K})\right) .
$$

(b) Existem seqüências $\left\{a_{n}\right\}_{n \in \mathbb{N}} \subset \mathbb{N} e\left\{S_{n}\right\}_{n \in \mathbb{N}} \subset \mathcal{S}_{f}$ tais que

(i) $S_{n} \supset S_{n+1}, a_{n}<a_{n+1} e \lim _{n \rightarrow \infty} a_{n}=\infty$;

(ii) $x \mathcal{X}_{S_{n}} \underset{n \rightarrow \infty}{\longrightarrow} 0$;

(iii) $\left|x \mathcal{X}_{S_{n}}\right|<\dot{\alpha}_{a_{n}}$.

Prova. Suponhamos que $x$ não satisfaz (a) e vamos mostrar que $x$ satisfaz (b). Como $x \in \overline{\mathbb{K}}_{f} \backslash\{0\}$ e $x \notin \operatorname{Inv}\left(\overline{\mathbb{K}}_{f}\right)$ segue pela Proposição 1.3 .16 que existe $a_{1} \in \mathbb{N}$ tal que se $S_{1}:=N_{a_{1}}(x)$, então $S_{1} \in \mathcal{S}_{f}$ e $\left|x \mathcal{X}_{S_{1}}\right|<\dot{\alpha}_{a_{1}}$. Seja $x_{2}=x \mathcal{X}_{S_{1}}$. Então $x_{2} \neq 0$, pois se $x_{2}=0$, então $x \mathcal{X}_{S_{1}}=0$, isto é, $x$ satisfaz $(a)(i)$, mas pela Definição 1.3 .13 de $N_{a_{1}}(x)$ temos também que $\left|x \mathcal{X}_{S_{1}^{c}}\right| \geq \dot{\alpha}_{a_{1}} \mathcal{X}_{S_{1}^{c}}$ e, portanto, $x$ satisfaz $(a)$ (ii). Logo, $x$ satisfaz (a) o que é um absurdo, pois, por hipótese, temos que $x$ não satisfaz $(a)$. Agora, $x_{2} \mathcal{X}_{S_{1}^{c}}=0$, mas $\mathcal{X}_{S_{1}^{c}} \neq 0$, pois se $S_{1} \in \mathcal{S}_{f}$, então $S_{1}^{c} \in \mathcal{S}_{f}$ e, portanto, $x_{2}$ é um divisor de zero o que implica $x_{2} \notin \operatorname{Inv}\left(\overline{\mathbb{K}}_{f}\right)$. Temos então que $x_{2}$ está sobre as hipóteses da Proposição 1.3.16. Assim, existe $a_{2} \in \mathbb{N}$ tal que se $\tilde{S}_{2}:=N_{a_{2}}\left(x_{2}\right)$, então $\tilde{S}_{2} \in \mathcal{S}_{f}$ e $\left|x_{2} \mathcal{X}_{\tilde{S}_{2}}\right|<\dot{\alpha}_{a_{2}} \Rightarrow\left|x \mathcal{X}_{S_{1} \cap \tilde{S}_{2}}\right|<\dot{\alpha}_{a_{2}}$. Seja $S_{2}:=S_{1} \cap \tilde{S}_{2}$. Então $S_{2} \subset S_{1} \in \mathcal{S}_{f} \Rightarrow S_{2} \in \mathcal{S}_{f}$ e $\left|x \mathcal{X}_{S_{2}}\right|<\dot{\alpha}_{a_{2}}$. Agora, pela Definição 1.3.13 de $N_{a_{2}}\left(x_{2}\right)$ temos que

$$
\dot{\alpha}_{a_{2}} \leq\left|x_{2} \mathcal{X}_{\tilde{S}_{2}^{c}}\right|=\left|x \mathcal{X}_{S_{1} \cap \tilde{S}_{2}^{c}}\right|=\left|x \mathcal{X}_{\tilde{S}_{2}^{c}}\right|
$$


pois $\tilde{S}_{2}^{c} \subset S_{1}$. De fato, se $\varphi \in \tilde{S}_{2}^{c}$, então $\dot{\alpha}_{a_{2}}(\varphi) \leq\left|x_{2}(\varphi)\right|=\left|x(\varphi) \mathcal{X}_{S_{1}}(\varphi)\right|$ e sendo $\dot{\alpha}_{a_{2}}=(i(\varphi))^{a_{2}}>0$ temos que $\mid x(\varphi) \mathcal{X}_{S_{1}}\left(\varphi \mid>0 \Rightarrow \mathcal{X}_{S_{1}}(\varphi) \neq 0\right.$, isto é, $\mathcal{X}_{S_{1}}(\varphi)=1 \Rightarrow \varphi \in S_{1}$ e, portanto, $\tilde{S}_{2}^{c} \subset S_{1}$ e $S_{1} \cap \tilde{S}_{2}^{c}=\tilde{S}_{2}^{c}$. Agora, para toda $\varphi \in \tilde{S}_{2}^{c} \subset S_{1}$ temos por (1.9) que

$$
\dot{\alpha}_{a_{2}}(\varphi) \leq\left|x_{2}(\varphi)\right|=|x(\varphi)|<\dot{\alpha}_{a_{1}}(\varphi)
$$

Daí, temos que $\dot{\alpha}_{a_{2}}(\varphi)<\dot{\alpha}_{a_{1}}(\varphi), \forall \varphi \in \tilde{S}_{2}^{c}$ o que implica

$$
(i(\varphi))^{\boldsymbol{a}_{2}}<(i(\varphi))^{\boldsymbol{a}_{1}}, \forall \varphi \in \tilde{S}_{2}^{c}
$$

E, portanto, $a_{1}<a_{2}$ para alguma $\varphi \in \tilde{S}_{2}^{c}$ tal que $0<i(\varphi)<1$. Tal $\varphi \in \tilde{S}_{2}^{c}$ sempre existe, pois $\tilde{S}_{2}^{c} \in \mathcal{S}_{f}{ }^{4}$.

Agora, usando os mesmos argumentos usados para obter $S_{n}$ e $a_{n}(n=1,2)$ podemos construir indutivamente as seqüências $\left\{S_{n}\right\}_{n \in \mathbb{N}}$ e $\left\{a_{n}\right\}_{n \in \mathbb{N}}$ como em (b) verificando as condições $(i)$ e (iii). Faltando, portanto, mostrar que a condição (ii), se verifica. De fato, de (iii) segue que para cada $n \in \mathbb{N},\left|\left(x \mathcal{X}_{S_{n}}\right)(\varphi)\right|<\dot{\alpha}_{a_{n}}(\varphi), \forall \varphi \in \mathcal{A}_{0}(\mathbb{K}) \Rightarrow\left\|x \mathcal{X}_{S_{n}}\right\| \leq$ $\left\|\dot{\alpha}_{a_{n}}\right\|=e^{-a_{n}} \underset{n \rightarrow \infty}{\longrightarrow} 0$, pois por $(i)$ temos que $a_{n} \underset{n \rightarrow \infty}{\longrightarrow} \infty$ e, portanto, $x \mathcal{X}_{S_{n}} \underset{n \rightarrow \infty}{\longrightarrow} 0$, isto é, vale (ii). E, portanto, segue o resultado.

Teorema 1.3.18. $x \in \operatorname{Inv}\left(\overline{\mathbb{K}}_{f}\right)$ se, e somente se, existem $\left.\left.r>0, \tau: \mathcal{A}_{0}(\mathbb{K}) \rightarrow\right] 0,1\right]$ tal que

$$
\left|\hat{x}\left(\varphi_{\varepsilon}\right)\right| \geq \dot{\alpha}_{r}\left(\varphi_{\varepsilon}\right), \forall 0<\varepsilon<\tau(\varphi),
$$

onde $\hat{x}$ é um representante de $x$.

Prova. $(\Rightarrow)$ Suponhamos que $x \in \operatorname{Inv}(\overline{\mathbb{K}})$. Então pela recíproca do Lema 1.3 .15 (ii) temos que $\mathcal{X}_{N_{q}(\hat{x})}=0, \forall q \in \in \mathbb{N}$, i.e., $\hat{\mathcal{X}}_{N_{q}(\hat{x})} \in \mathcal{N}_{f}$. Pelo Lema 1.3.14 temos que existe $\left.\left.\tau: \mathcal{A}_{0}(\mathbb{K}) \rightarrow\right] 0,1\right]$ tal que para toda $\varphi \in \mathcal{A}_{q}(\mathbb{K})$ tem-se que $I_{\tau(\varphi)} \subset\left\{\varepsilon \mid \varphi_{\varepsilon} \in\left(N_{q}(\hat{x})\right)^{c}\right\}$ e, pela Definição 1.3 .13 , segue que

$$
\left|\hat{x}\left(\varphi_{\varepsilon}\right)\right| \geq \dot{\alpha}_{q}\left(\varphi_{\varepsilon}\right), \forall 0<\varepsilon<\tau(\varphi),
$$

onde $\hat{x}$ é um representante de $x$ e, portanto, basta tomarmos $q=r$.

$(\Leftarrow)$ Com efeito, se existem $\left.\left.r>0, \tau: \mathcal{A}_{0}(\mathbb{K}) \rightarrow\right] 0,1\right]$ tal que

$$
\left|\hat{x}\left(\varphi_{\varepsilon}\right)\right| \geq \dot{\alpha}_{r}\left(\varphi_{\varepsilon}\right), \forall 0<\varepsilon<\tau(\varphi),
$$

\footnotetext{
${ }^{4}$ De fato, tome $\varphi \in \tilde{S}_{2}^{c}$, então $i\left(\varphi_{\varepsilon}\right)=\varepsilon i(\varphi)$ e $\left\{\varepsilon \mid \varphi_{\varepsilon} \in \tilde{S}_{2}^{c}\right\} \in \mathcal{S}$, portanto existe $\varepsilon_{0}$ tal que $\varphi_{\varepsilon_{0}} \in \tilde{S}_{2}^{c}$ e $i\left(\varphi_{\varepsilon_{0}}\right)<1$, isto é, $\varepsilon_{0} i(\varphi)<1$, pois $i\left(\varphi_{\varepsilon}\right)=\varepsilon i(\varphi) \rightarrow 0$ quando $\varepsilon \rightarrow 0$.
} 
então

$$
\left|\hat{x}\left(\varphi_{\varepsilon}\right)\right| \geq(i(\varphi))^{r} \varepsilon^{r}, \forall 0<\varepsilon<\tau(\varphi)
$$

e, portanto,

$$
\left|\frac{1}{\hat{x}\left(\varphi_{\varepsilon}\right)}\right| \leq(i(\varphi))^{-r} \varepsilon^{-r}, \forall 0<\varepsilon<\tau(\varphi) .
$$

Afirmamos que $1 / \hat{x} \in \mathcal{E}_{f}^{M}(\mathbb{K})$. Para mostrarmos isto, pela Definição 1.1.5 das moderadas, precisamos mostrar que existe $p \in \mathbb{N}$ tal que $\forall \varphi \in \mathcal{A}_{p}(\mathbb{K})$ existem $C=C(\varphi)>0, \eta=$ $\eta(\varphi)>0$ tal que

$$
\left|\frac{1}{\hat{x}\left(\varphi_{\varepsilon}\right)}\right| \leq C \varepsilon^{-p}, \forall 0<\varepsilon<\eta .
$$

Basta então tomarmos $p=r>0, C=C(\varphi)=(i(\varphi))^{-p}>0$ e $\eta=\eta(\varphi)=\tau(\varphi)>0$ para obtermos,

$$
\left|\frac{1}{\hat{x}\left(\varphi_{\varepsilon}\right)}\right| \leq(i(\varphi))^{-r} \varepsilon^{-r}=C \varepsilon^{-p}, \forall 0<\varepsilon<\tau(\varphi) .
$$

$\mathrm{E}$, portanto, a afirmação está provada e $x \in \operatorname{Inv}\left(\overline{\mathbb{K}}_{f}\right)$, pois $\hat{x} 1 / \hat{x}=1$, onde $\hat{x}$ e $1 / \hat{x}$ são representantes de $x$ e $1 / x$, respectivamente.

Lema 1.3.19. Seja $x \in \overline{\mathbb{K}}_{f} \backslash\{0\}$ uma não-unidade. Então existe $a \in \mathbb{N}$ tal que

$$
y:=x\left(1-\mathcal{X}_{N_{a}(\hat{x})}\right)+\mathcal{X}_{N_{a}(\hat{x})} \in \operatorname{Inv}\left(\overline{\mathbb{K}}_{f}\right) .
$$

Prova. Suponhamos que $x \in \overline{\mathbb{K}}_{f} \backslash\{0\}$ é uma não-unidade. Então pela Proposição 1.3.16 existe $a \in \mathbb{N}$ tal que $N_{a}(\hat{x}) \in \mathcal{S}_{f}$ e, portanto, $\mathcal{X}_{N_{a}(\hat{x})} \neq 0$ e $\mathcal{X}_{N_{a}(\hat{x})} \neq 1$. Seja

$$
\hat{y}:=\hat{x}\left(1-\hat{\mathcal{X}}_{N_{a}(\hat{x})}\right)+\hat{\mathcal{X}}_{N_{a}(\hat{x})} \text {. }
$$

Então é suficiente mostrarmos que $\left|\hat{y}\left(\varphi_{\varepsilon}\right)\right| \geq \dot{\alpha}_{a}\left(\varphi_{\varepsilon}\right), \forall 0<\varepsilon \leq 1$. Para todo $0<\varepsilon \leq 1$, temos

$$
\hat{y}\left(\varphi_{\varepsilon}\right)= \begin{cases}1, & \text { se } \varphi_{\varepsilon} \in N_{a}(\hat{x}) \\ \hat{x}\left(\varphi_{\varepsilon}\right), & \text { se } \varphi_{\varepsilon} \in\left(N_{a}(\hat{x})\right)^{c}\end{cases}
$$

E, portanto, para todo $0<\varepsilon \leq 1$, temos

$$
\left|\hat{y}\left(\varphi_{\varepsilon}\right)\right|= \begin{cases}1, & \text { se } \varphi_{\varepsilon} \in N_{a}(\hat{x}) \\ \left|\hat{x}\left(\varphi_{\varepsilon}\right)\right|, & \text { se } \varphi_{\varepsilon} \in\left(N_{a}(x)\right)^{c}\end{cases}
$$

Logo, pela Definição 1.3.13 de $N_{a}(\hat{x})$, temos que $\left|\hat{y}\left(\varphi_{\varepsilon}\right)\right| \geq \dot{\alpha}_{a}\left(\varphi_{\varepsilon}\right)$ para todo $0<\varepsilon \leq 1$, pois $\left|\hat{x}\left(\varphi_{\varepsilon}\right)\right| \geq \dot{\alpha}_{a}\left(\varphi_{\varepsilon}\right)$ já que $\varphi_{\varepsilon} \notin N_{a}(\hat{x}), \forall 0<\varepsilon \leq 1$. Assim, pelo Teorema 1.3.18 segue que $\hat{y} \in \operatorname{Inv}\left(\overline{\mathbb{K}}_{f}\right)$ para todo representante $\hat{y}$ de $y$ e, portanto, $y \in \operatorname{Inv}\left(\overline{\mathbb{K}}_{f}\right)$. 
Teorema 1.3.20. Seja $x \in \overline{\mathbb{K}}_{f} \backslash\{0\}$ uma não-unidade. Então existe um ideal maximal $\mathfrak{m}$ de $\overline{\mathbb{K}}_{f}$ tal que $x \notin \mathfrak{m}$ e, portanto, o radical de Jacobson de $\overline{\mathbb{K}}_{f}$ denotado por $\operatorname{Rad}\left(\overline{\mathbb{K}}_{f}\right)$ é nulo, isto é, $\operatorname{Rad}\left(\overline{\mathbb{K}}_{f}\right)=\{0\}$.

Prova. Seja $x \in \overline{\mathbb{K}}_{f} \backslash\{0\}$ uma não-unidade. Então pelo Lema 1.3.19 existe $a \in \mathbb{N}$ tal que

$$
y=x\left(1-\mathcal{X}_{N_{a}(\hat{x})}\right)+\mathcal{X}_{N_{a}(\hat{x})} \in \operatorname{Inv}\left(\overline{\mathbb{K}}_{f}\right) .
$$

Agora, como $\mathcal{X}_{N_{a}(\hat{x})} \notin\{0,1\}$ e também $\mathcal{X}_{N_{a}(\hat{x})} \notin \operatorname{Inv}\left(\overline{\mathbb{K}}_{f}\right), \overline{\mathbb{K}}_{f}$ um anel com unidade, segue que existe $\mathfrak{m} \triangleleft \overline{\mathbb{K}}_{f}$ ideal maximal tal que $\mathcal{X}_{N_{a}(\hat{x})} \in \mathfrak{m}$. Agora, veremos que $x \notin \mathfrak{m}$. De fato, se $x \in \mathfrak{m}$, então $x \mathcal{X}_{\left(N_{a}(\hat{x})\right)^{c}}=x\left(1-\mathcal{X}_{N_{a}(\hat{x})}\right) \in \mathfrak{m}$ e, portanto, $y=x\left(1-\mathcal{X}_{N_{a}(\hat{x})}\right)+\mathcal{X}_{N_{a}(\hat{x})} \in \mathfrak{m}$. Como $y \in \operatorname{Inv}\left(\overline{\mathbb{K}}_{f}\right)$ segue que $y \in \mathfrak{m} \cap \operatorname{Inv}\left(\overline{\mathbb{K}}_{f}\right)$ o que implica $\mathfrak{m}=\overline{\mathbb{K}}_{f}$ o que é um absurdo. Logo, $x \notin \mathrm{m}$. Agora, se

$$
x \in \operatorname{Rad}\left(\overline{\mathbb{K}}_{f}\right)=\bigcap_{\mathfrak{M} \triangleleft \overline{\mathbb{K}}_{f}} \mathfrak{M},
$$

então $x \in \mathfrak{M}, \forall \mathfrak{M} \triangleleft \overline{\mathbb{K}}_{f}$ ideal maximal. Assim, se $x \neq 0$, então pelo que acabamos de mostrar existe $\mathfrak{m} \triangleleft \overline{\mathbb{K}}_{f}$ ideal maximal tal que $x \notin \mathfrak{m}$, absurdo! Logo, $x=0$, isto é, $\operatorname{Rad}\left(\overline{\mathbb{K}}_{f}\right)=\{0\}$.

Seja $\hat{\mathbb{R}}:=\left\{r: \mathcal{A}_{0}(\mathbb{K}) \rightarrow \mathbb{R} \mid r\right.$ é limitada $\}$ ou seja $\hat{\mathbb{R}}=\mathcal{B}\left(\mathcal{A}_{0}(\mathbb{K}), \mathbb{R}\right)$. Para $r \in \hat{\mathbb{R}}$ defina

$$
\hat{\dot{\alpha}}_{r}: \mathcal{A}_{0}(\mathbb{K}) \rightarrow \mathbb{R}
$$

por

$$
\hat{\dot{\alpha}}_{r}(\varphi):=[i(\varphi)]^{r(\varphi)} .
$$

Note que quando $r(\varphi)=r_{0}$, constante, obtemos a função $\hat{\dot{\alpha}}_{r_{0}}=\dot{\alpha}_{r_{0}}$ já conhecida. Observe também que

$$
\begin{aligned}
\hat{\dot{\alpha}}_{r}\left(\varphi_{\varepsilon}\right) & =\left[i\left(\varphi_{\varepsilon}\right)\right]^{r\left(\varphi_{\varepsilon}\right)} \\
& =[i(\varphi)]^{r\left(\varphi_{\varepsilon}\right)} \varepsilon^{r\left(\varphi_{\varepsilon}\right)} .
\end{aligned}
$$

Sendo $r \in \hat{\mathbb{R}} \Leftrightarrow \exists C$ tal que $0 \leq[i(\varphi)]^{r\left(\varphi_{\varepsilon}\right)} \leq C$. Portanto, $\left|\hat{\dot{\alpha}}_{r}\left(\varphi_{\varepsilon}\right)\right| \leq C \varepsilon^{r\left(\varphi_{\varepsilon}\right)}, \exists a \in \mathbb{R}$ tal que $a \leq r\left(\varphi_{\varepsilon}\right), \forall \varepsilon$. Dai, como $0<\varepsilon \leq 1 \Rightarrow \varepsilon^{r\left(\varphi_{\varepsilon}\right)}<\varepsilon^{a}$. E, portanto,

$$
\left|\hat{\dot{\alpha}}_{r}\left(\varphi_{\varepsilon}\right)\right| \leq C \varepsilon^{q}
$$

para algum $q \in \mathbb{N}$. 
A partir do conjunto $\hat{\mathbb{R}}$, obtemos uma nova caracterização para os invertíveis de $\overline{\mathbb{K}}_{f}$.

Vamos agora enunciar e demonstrar o principal resultado deste capítulo como haviámos prometido, a saber:

Teorema 1.3.21. (i) Para todo ideal maximal $\mathfrak{m}$ de $\overline{\mathbb{K}}_{f}$ temos que

$$
\mathfrak{m}=\overline{g_{f}\left(\mathcal{F}_{\mathfrak{m}}\right)}
$$

(ii) Para todo $\mathcal{F} \in P_{*}\left(\mathcal{S}_{f}\right)$ o ideal $\mathfrak{m}:=\overline{g_{f}\left(\mathcal{F}_{\mathfrak{m}}\right)}$ é maximal e $\mathcal{F}=\mathcal{F}_{\mathfrak{m}}$.

Prova. (i) Seja $m$ um ideal maximal de $\overline{\mathbb{K}}_{f}$. Então $\mathfrak{m}$ é um ideal primo de $\overline{\mathbb{K}}_{f}$ e pelo Teorema da aproximação existe um único $\mathcal{F}=\mathcal{F}_{\mathfrak{m}}$ tal que $g_{f}(\mathcal{F}) \subset \mathfrak{m}$. Seja $x \in \mathfrak{m} \backslash g_{f}(\mathcal{F})$. Vamos construir uma seqüência $\left\{x_{n}\right\}_{n \in \mathbb{N}}$ em $g_{f}(\mathcal{F})$ tal que $x_{n} \underset{n \rightarrow \infty}{\longrightarrow} x$ em $\overline{\mathbb{K}}_{f}$. Para isto vamos mostrar que $x$ satisfaz a condição $(b)$ do Teorema da Aproximação. De fato, se $x$ não satisfaz a condição $(b)$ do Teorema da aproximação, então, temos que $x$ satisfaz a condição (a) do Teorema da aproximação, isto é, existem $S \in \mathcal{S}_{f}$ e $a \in \mathbb{N}$ tal que

(i) $x \mathcal{X}_{S}=0$

(ii) $\left|x \mathcal{X}_{S^{c}}\right| \geq \dot{\alpha}_{a} \mathcal{X}_{S^{c}}$

Lembre-se que este $S$ é exatamente $N_{a}(x)$. Suponhamos que $S \in \mathcal{F}$. Então

$$
\mathcal{X}_{S} \in g_{f}(\mathcal{F}) \subset \mathfrak{m} \Rightarrow \mathcal{X}_{S} \in \mathfrak{m}
$$

Logo,

$$
y:=x\left(1-\mathcal{X}_{S}\right)+\mathcal{X}_{S} \in \mathfrak{m}
$$

o que é um absurdo, pois pelo Lema 1.3.19 y é invertível. Agora, suponhamos que $S^{c} \in \mathcal{F}$. Então $\mathcal{X}_{S^{c}}=\left(1-\mathcal{X}_{S}\right) \in g_{f}(\mathcal{F})$ e como por $(a)$ parte $(i) x \mathcal{X}_{S}=0$ segue que

$$
x \mathcal{X}_{S^{\mathrm{c}}}=x\left(1-\mathcal{X}_{S}\right)=x \in g_{f}(\mathcal{F})
$$

o que é um absurdo, pois, por hipótese, $x \notin g_{f}(\mathcal{F})$. Logo, concluímos que $x$ não pode satisfazer a condição $(a)$ do Teorema da Aproximação e, portanto, $x$ satisfaz a condição (b) do Teorema da Aproximação, i.e., existem seqüências $\left\{a_{n}\right\} \subset \mathbb{N}$ e $\left\{S_{n}\right\} \subset \mathcal{S}_{f}$ tais que 
(i) $S_{n} \supset S_{n+1}, a_{n}<a_{n+1} \mathrm{e}$

$$
\lim _{n \rightarrow \infty} a_{n}=\infty
$$

(ii) $x \mathcal{X}_{S_{n}} \underset{n \rightarrow \infty}{\longrightarrow} 0$;

(iii) $\left|x \mathcal{X}_{S_{n}}\right|<\dot{\alpha}_{a_{n}}$

Afirmamos que $S_{n}^{c} \in \mathcal{F}$. De fato, se isto não for o caso, então $S_{n} \in \mathcal{F}$ e, portanto,

$$
\mathcal{X}_{S_{n}} \in g_{f}(\mathcal{F}) \subset \mathfrak{m} \Rightarrow \mathcal{X}_{S_{n}} \in \mathfrak{m}
$$

e mais uma vez temos que

$$
y=x\left(1-\mathcal{X}_{S_{n}}\right)+\mathcal{X}_{S_{n}} \in \mathfrak{m}
$$

o que é um absurdo, pois pelo Lema $1.3 .19 y \in \operatorname{Inv}\left(\overline{\mathbb{K}}_{f}\right)$. Logo, $S_{n}^{c} \in \mathcal{F}$ o que implica $\mathcal{X}_{S_{n}^{c}} \in g_{f}(\mathcal{F}), \operatorname{mas} \mathcal{X}_{S_{n}^{c}}=1-\mathcal{X}_{S_{n}}$ e, portanto,

$$
x_{n}=x \mathcal{X}_{S_{n}^{c}}=x-x \mathcal{X}_{S_{n}}
$$

é uma seqüência em $g_{f}(\mathcal{F})$ tal que $x_{n} \underset{n \rightarrow \infty}{\longrightarrow} x$, pois por $(i i)$ de $(b)$ do Teorema da aproximação temos que $x \mathcal{X}_{S_{n}} \underset{n \rightarrow \infty}{\longrightarrow} 0$. E, portanto, $\mathrm{m}=\overline{g_{f}\left(\mathcal{F}_{\mathrm{m}}\right)}$.

(ii) Pelo Lema 1.3.8 $g_{f}(\mathcal{F})$ é um ideal próprio de $\overline{\mathbb{K}}_{f}$ e, portanto, $g_{f}(\mathcal{F}) \subset \mathfrak{m}$ para algum ideal maximal $\mathrm{m}$ de $\overline{\mathbb{K}}_{f}$. Mais ainda, pelo Teorema da aproximação temos que $\mathcal{F}=\mathcal{F}_{\mathrm{m}}$ e a conclusão vem imediatamente de $(i)$.

O próximo resultado é mais uma aplicação do Teorema da Aproximação.

Teorema 1.3.22. $\operatorname{Inv}\left(\overline{\mathbb{K}}_{f}\right)$ é um subconjunto aberto e denso de $\overline{\mathbb{K}}_{f}$.

Prova. Sabemos do Corolário 1.2.3 que $\operatorname{Inv}\left(\overline{\mathbb{K}}_{f}\right)$ é um subconjunto aberto de $\overline{\mathbb{K}}_{f}$, portanto, resta mostrarmos que $\operatorname{Inv}\left(\overline{\mathbb{K}}_{f}\right)$ é um subconjunto denso de $\overline{\mathbb{K}}_{f}$. Isto é, todo elemento $x \in \overline{\mathbb{K}}_{f}$ é limite de alguma seqüência de elementos de $\operatorname{Inv}\left(\overline{\mathbb{K}}_{f}\right)$. Pelo Lema 1.2.5 $(i)$, temos que $0 \in \overline{\mathbb{K}}_{f}$ é limite de uma seqüência em $\operatorname{Inv}\left(\overline{\mathbb{K}}_{f}\right)^{5}$. Basta então estudarmos o caso em que $x \in \overline{\mathbb{K}}_{f} \backslash\{0\}$ e $x \notin \operatorname{Inv}\left(\overline{\mathbb{K}}_{f}\right)$, pois se $x \in \operatorname{Inv}\left(\overline{\mathbb{K}}_{f}\right)$ tomamos a seqüência constante. Pelo Teorema da aproximação $x$ satisfaz (a) ou $x$ satisfaz (b). Em cada um dos casos vamos

\footnotetext{
${ }^{5}$ Veja a seqüência $\left\{\dot{\alpha}_{n}\right\}_{n \in \mathbb{N}}$ em $\operatorname{Inv}\left(\overline{\mathbb{K}}_{f}\right)$ que é $\tau_{s f}$-convergente a zero.
} 
construir seqüências em $\operatorname{Inv}\left(\overline{\mathbb{K}}_{f}\right)$ que são $\tau_{s f}$-convergente a $x$. Inicialmente suponhamos que $x$ satisfaz $(a)$ e defina

$$
x_{n}:=x\left(1-\mathcal{X}_{S}\right)+\dot{\alpha}_{n} \mathcal{X}_{S}
$$

Vamos mostrar que $x_{n} \in \operatorname{Inv}\left(\overline{\mathbb{K}}_{f}\right), \forall n \in \mathbb{N}$ e que $x_{n} \underset{n \rightarrow \infty}{\longrightarrow} x$. De fato, pelo o item (i) de (a), temos que

$$
x_{n}=x+\dot{\alpha}_{n} \mathcal{X}_{S}, \text { pois } x \mathcal{X}_{S}=0 .
$$

Agora, lembrando que $S=N_{a}(x)$ e

$$
x_{n}(\varphi)= \begin{cases}x(\varphi)+\dot{\alpha}_{n}(\varphi), & \text { se } \varphi \in S \\ x(\varphi), & \text { se } \varphi \in S^{c}\end{cases}
$$

temos que

$$
\left|x_{n}(\varphi)\right| \geq \dot{\alpha}_{n}(\varphi), \forall \varphi \text { e } \forall n \in \mathbb{N}
$$

Pelo Teorema 1.3.18, $x_{n} \in \operatorname{Inv}\left(\overline{\mathbb{K}}_{f}\right), \forall n \in \mathbb{N}$, i.e., $\left\{x_{n}\right\}_{n \in \mathbb{N}}$ é uma seqüência em $\operatorname{Inv}\left(\overline{\mathbb{K}}_{f}\right)$. Agora, como

$$
x_{n}=x+\dot{\alpha}_{n} \mathcal{X}_{S}
$$

segue que

$$
x_{n}-x=\dot{\alpha}_{n} \mathcal{X}_{S}
$$

E, portanto, pelo Corolário 1.1.12 (iv), temos

$$
\left\|x_{n}-x\right\|=\left\|\dot{\alpha}_{n} \mathcal{X}_{S}\right\|=e^{-n}\left\|\mathcal{X}_{S}\right\|=e^{-n}
$$

i.e.,

$$
\left\|x_{n}-x\right\|=e^{-n}
$$

e como $e^{-n} \underset{n \rightarrow \infty}{\longrightarrow} 0$ segue que $\left\|x_{n}-x\right\| \underset{n \rightarrow \infty}{\longrightarrow} 0$ o que implica $x_{n} \underset{n \rightarrow \infty}{\longrightarrow} x$. Por último, suponhamos que $x$ satisfaz a condição $(b)$ e defina

$$
x_{n}:=x\left(1-\mathcal{X}_{S_{n}}\right)+\dot{\alpha}_{a_{n}} \mathcal{X}_{S_{n}}
$$

Vamos mostrar que $x_{n} \in \operatorname{Inv}\left(\overline{\mathbb{K}}_{f}\right), \forall n \in \mathbb{N}$ e que $x_{n} \underset{n \rightarrow \infty}{\longrightarrow} x$. De fato,

$$
x_{n}(\varphi)= \begin{cases}\dot{\alpha}_{a_{n}}(\varphi), & \text { se } \varphi \in S_{n} \\ x(\varphi), & \text { se } \varphi \in S_{n}^{c}\end{cases}
$$


Relembrando que na demonstração do Teorema da aproximação $S_{n}=N_{a_{n}}(x)$, temos que

$$
\left|x_{n}(\varphi)\right| \geq \dot{\alpha}_{a_{n}}(\varphi), \forall \varphi, \forall n \in \mathbb{N}
$$

E, mais uma vez, pelo Teorema 1.3.18, vem $x_{n} \in \operatorname{Inv}\left(\overline{\mathbb{K}}_{f}\right), \forall n \in \mathbb{N}$. Como

$$
x_{n}=x-x \mathcal{X}_{S_{n}}+\dot{\alpha}_{a_{n}} \mathcal{X}_{S_{n}}
$$

segue que

$$
\begin{aligned}
\left\|x_{n}-x\right\| & =\left\|\dot{\alpha}_{a_{n}} \mathcal{X}_{S_{n}}-x \mathcal{X}_{S_{n}}\right\| \\
& \leq\left\|\dot{\alpha}_{a_{n}} \mathcal{X}_{S_{n}}+x \mathcal{X}_{S_{n}}\right\| \text { (pelo Corolário 1.1.12(i), temos) } \\
& \leq \max \left\{\left\|\dot{\alpha}_{a_{n}} \mathcal{X}_{S_{n}}\right\|,\left\|x \mathcal{X}_{S_{n}}\right\|\right\} \text { (pelo Corolário 1.1.12(iv), temos) } \\
& =\max \left\{e^{-a_{n}},\left\|x \mathcal{X}_{S_{n}}\right\|\right\} .
\end{aligned}
$$

Mas pelos itens $(i)$ e $(i i)$ de $(b)$, temos que $\max \left\{e^{-a_{n}},\left\|x \mathcal{X}_{S_{n}}\right\|\right\} \underset{n \rightarrow \infty}{\longrightarrow} 0 \Rightarrow\left\|x_{n}-x\right\| \underset{n \rightarrow \infty}{\longrightarrow} 0$ e, portanto, $x_{n} \underset{n \rightarrow \infty}{\longrightarrow} x$. Isto mostra nossa assertiva.

Para terminarmos este capítulo temos a seguinte:

Observação 1.3.23. (i) Se $\mathcal{F}_{1}, \mathcal{F}_{2} \in P_{*}\left(\mathcal{S}_{f}\right)$ e $\mathcal{F}_{1} \neq \mathcal{F}_{2}$, então $g_{f}\left(\mathcal{F}_{1}\right)+g_{f}\left(\mathcal{F}_{2}\right)=\overline{\mathbb{K}}_{f}$. Com efeito, se $\mathcal{F}_{1} \neq \mathcal{F}_{2}$, então existe $S \in \mathcal{F}_{1}$ tal que $S \notin \mathcal{F}_{2}$. Assim, $S^{c} \in \mathcal{F}_{2}$ e, portanto, $1=\mathcal{X}_{S}+\mathcal{X}_{S^{c}} \in g_{f}\left(\mathcal{F}_{1}\right)+g_{f}\left(\mathcal{F}_{2}\right)$, pois $\mathcal{X}_{S} \in g_{f}\left(\mathcal{F}_{1}\right)$ e $\mathcal{X}_{S^{c}} \in g_{f}\left(\mathcal{F}_{2}\right)$ o que mostra que $\overline{\mathbb{K}}_{f}=g_{f}\left(\mathcal{F}_{1}\right)+g_{f}\left(\mathcal{F}_{2}\right)$.

(ii) Se $\mathcal{F} \in P_{*}\left(\mathcal{S}_{f}\right)$, então $\overline{\mathbb{K}}_{f} / g_{f}(\mathcal{F})$ é um anel local cujo o ideal maximal é $\overline{g_{f}(\mathcal{F})} / g_{f}(\mathcal{F})$. Defina $B:=\overline{\mathbb{K}}_{f} / g_{f}(\mathcal{F})$ e denote por $\varphi: \overline{\mathbb{K}}_{f} \rightarrow B$ a aplicação quociente. Seja, então $\mathfrak{m}_{0}$ um ideal maximal de $B$. Então $\mathfrak{m}:=\varphi^{-1}\left(\mathfrak{m}_{0}\right)$ é um ideal maximal de $\overline{\mathbb{K}}_{f}$ contendo o ideal $g_{f}(\mathcal{F})$. Portanto, pelo Teorema 1.3.21 segue que $\mathcal{F}=\mathcal{F}_{\mathfrak{m}}$ e $\mathfrak{m}=\overline{g_{f}(\mathcal{F})}$. Logo, temos que

$$
\mathfrak{m}_{0}=\varphi(\mathfrak{m})=\varphi\left(\overline{g_{f}(\mathcal{F})}\right)=\overline{g_{f}(\mathcal{F})} / g_{f}(\mathcal{F})
$$

é o ideal maximal de $B$.

(iii) $O$ corpo $\overline{\mathbb{K}}_{f} / \overline{g_{f}(\mathcal{F})}$ tem característica zero para todo $\mathcal{F} \in P_{*}\left(\mathcal{S}_{f}\right)$.

Fixemos qualquer $\mathcal{F} \in P_{*}\left(\mathcal{S}_{f}\right)$. Pelo Teorema 1.3.21 sabemos que $\mathrm{m}:=\overline{g_{f}(\mathcal{F})}$ é um ideal maximal de $\overline{\mathbb{K}}_{f}$. Portanto, pelo Teorema 1.2 .7 podemos concluir que $\overline{\mathbb{K}}_{f} / \mathfrak{m}$ é uma extensão do corpo $\mathbb{K}$. Portanto, a afirmação segue do fato que o corpo $\mathbb{K}$ tem característica zero. 
No Capítulo 2, damos mais algumas propriedades algébricas do anel dos números generalizados plenos de Colombeau $\overline{\mathbb{K}}_{f}$. 


\section{Relação de ordem sobre $\overline{\mathbb{R}}_{f}$ e outras propriedades algébricas de $\overline{\mathbb{K}}_{f}$}

Neste capítulo, vamos introduzir uma relação de ordem parcial sobre $\overline{\mathbb{R}}_{f}$, o anel dos números generalizados plenos de Colombeau (sobre os reais) que provaremos induzir uma relação de ordem total em todo o corpo de classe residual. A base para este capítulo são os trabalhos de Aragona, Juriaans, Oliveira e Scarpalézos [6] e Aragona, Fernandez e Juriaans [3] que desenvolveram pesquisas, semelhantes com o anel dos números generalizados simplificados de Colombeau $\overline{\mathbb{K}}$ (sobre $\mathbb{R}$ ou $\mathbb{C}$ ).

\subsection{Relação de ordem sobre $\overline{\mathbb{R}}_{f}$}

O lema abaixo devido a Aragona, Fernandez e Juriaans [3] é a base para a definição de ordem em $\overline{\mathbb{R}}_{f}$.

Lema 2.1.1. Para todo $x \in \overline{\mathbb{R}}_{f}$ as seguintes afirmações são equivalentes:

(i) Todo representante $\hat{x}$ de $x$ satisfaz a condição

$$
(*) \mid \begin{aligned}
& \exists N \in \mathbb{N} \text { tal que } \forall b>0 \forall \varphi \in \mathcal{A}_{N}(\mathbb{K}) \exists \\
& \eta=\eta(b, \varphi) \in I \text { tal que } \hat{x}\left(\varphi_{\varepsilon}\right) \geq-\varepsilon^{b}, \forall \varepsilon \in I_{\eta} ;
\end{aligned}
$$

(ii) existe um representante $\hat{x}$ de $x$ tal que $\hat{x}$ satisfaz $(*)$;

(iii) existe um representante $x_{*}$ de $x$ tal que $x_{*}(\varphi) \geq 0, \forall \varphi \in \mathcal{A}_{0}(\mathbb{K})$; 
(iv) existe $N \in \mathbb{N}$ e um representante $x_{*}$ de $x$ tal que $x_{*}(\varphi) \geq 0, \forall \varphi \in \mathcal{A}_{N}(\mathbb{K})$.

Prova. Ver [3].

Definição 2.1.2. Um elemento $x \in \overline{\mathbb{R}}_{f}$ é dito quase-positivo ou q-positivo, se tem um representante satisfazendo as condições equivalentes do Lema 2.1.1. Denotaremos isto por $x \geq 0$. Dizemos também que $x$ é quase-negativo ou q-negativo se $-x$ é q-positivo $e$ denotamos isto por $x \leq 0$. Se $y \in \overline{\mathbb{R}}_{f}$ é um outro elemento então escrevemos $x \leq y$ (resp. $x \geq y$ ) se $y-x$ (resp. $x-y)$ é q-positivo.

Definição 2.1.3 (Conjunto parcialmente ordenado, cadeia). Um conjunto parcialmente ordenado é um conjunto $M$ sobre o qual existe uma ordem parcial definida, isto é, uma relação binária que indicamos por $\leq$ e que satisfaz as seguintes condições:

(i) $a \leq a, \forall a \in M$ (Reflexiva);

(ii) se $a \leq b$ e $b \leq a$, então $a=b$ (Anti-simétrica);

(iii) Se $a \leq b$ e $b \leq c$, então $a \leq c$ (Transitiva).

"Parcial" significa que $M$ pode conter elementos a e b não comparáveis, isto é, não vale que $a \leq b$ ou $b \leq a$. E neste caso dizemos que $a$ e b são incomparáveis. Ao contrário, dois elementos a e b são chamados de elementos comparáveis se eles satisfazem: $a \leq b$ ou $b \leq a$ (ou ambos, e neste caso temos que $a=b$ ).

Um conjunto totalmente ordenado ou cadeia é um conjunto parcialmente ordenado tal que dois quaisquer elementos do conjunto são sempre comparáveis. Em outras palavras, uma cadeia é um conjunto parcialmente ordenado que não tem elementos incomparáveis.

Observação 2.1.4. A Definição 2.1.2 não define uma relação de ordem total sobre $\overline{\mathbb{R}}_{f}$, pois, por exemplo, o número generalizado $\hat{x}(\varphi)=\hat{\dot{\alpha}}_{1}(\varphi) \sin \left(\hat{\dot{\alpha}}_{-1}(\varphi)\right), \forall \varphi \in \mathcal{A}_{0}(\mathbb{K})$ não é q-positivo nem q-negativo. Entretanto ela define uma relação de ordem parcial tal que a soma e o produto de elementos q-positivos são q-positivos.

Para todo $x \in \overline{\mathbb{K}}_{f}$, se $\hat{x}$ é um representante de $x$, a função

$$
|\hat{x}|: \mathcal{A}_{0} \rightarrow \mathbb{R}_{+}
$$


definida por

$$
|\hat{x}|(\varphi)=|\hat{x}(\varphi)|
$$

é naturalmente uma função moderada e $|x|:=\operatorname{cl}[|\hat{x}|]$ é independente do representante $\hat{x}$ de $x$. Este número generalizado pleno de Colombeau é chamado o valor absoluto de $x$. Assim, temos a aplicação natural $x \in \overline{\mathbb{K}}_{f} \mapsto|x| \in \overline{\mathbb{R}}_{f+}$

Definição 2.1.5. Seja $x \in \overline{\mathbb{R}}_{f}$. Então $x^{+}:=\frac{x+|x|}{2}$ e $x^{-}:=\frac{x-|x|}{2}$ são chamados respectivamente de parte q-positiva e q-negativa de $x$.

Note que $x^{+}$e $x^{-}$dependem apenas de $x$.

Definição 2.1.6. Dado $u \in \mathcal{E}_{f}^{M}(\mathbb{K})$ definimos as seguintes funções

$$
\begin{gathered}
\theta_{u}: \mathcal{A}_{0}(\mathbb{K}) \rightarrow \mathbb{K} \\
\theta_{u}(\varphi)=\exp (-i \operatorname{Arg}(u(\varphi)))
\end{gathered}
$$

$e$

$$
\begin{gathered}
\theta_{u}^{-1}: \mathcal{A}_{0}(\mathbb{K}): \rightarrow \mathbb{K} \\
\theta_{u}^{-1}(\varphi)=\exp (i \operatorname{Arg}(u(\varphi))),
\end{gathered}
$$

onde $\operatorname{Arg}(u(\varphi))$ denota o argumento de $u(\varphi) \in \mathbb{K}$, com a convenção que $\operatorname{Arg}(0):=0$.

No caso em que $\mathbb{K}=\mathbb{R}$ as imagens de $\theta_{u}$ e $\theta_{u}^{-1}$ pertencem ao conjunto $\{-1,1\}$. É claro também que estas funções são moderadas e uma é a inversa da outra $\left(\theta_{u} \theta_{u}^{-1}=\right.$ $\left.1=\theta_{u}^{-1} \theta_{u}\right)$. Mais ainda, $u(\varphi) \theta_{u}(\varphi)=|u(\varphi)|, \forall \varphi \in \mathcal{A}_{0}(\mathbb{K})$. Portanto, se denotamos $|u|(\varphi):=|u(\varphi)|, \forall \varphi \in \mathcal{A}_{0}(\mathbb{K})$, podemos escrever $u \theta_{u}=|u|$ e, portanto, $|u| \in \mathcal{E}_{f}^{M}(\mathbb{R})$.

Definição 2.1.7. Dado $u \in \mathcal{E}_{f}^{M}(\mathbb{K})$ definimos $\Theta_{u}=\operatorname{cl}\left[\theta_{u}\right]$ e $\Theta_{u}^{-1}=\operatorname{cl}\left[\theta_{u}^{-1}\right]$

Como $\Theta_{u} \Theta_{u}^{-1}=1=\Theta_{u}^{-1} \Theta_{u}$ segue que $\Theta_{u}, \Theta_{u}^{-1} \in \operatorname{Inv}(\mathbb{K})$. Note entretanto, que estas funções dependem do representante.

Proposição 2.1.8. Seja $x, y \in \overline{\mathbb{R}}_{f}$. Então:

(i) $x=x^{+}$se, e somente se, $x=|x|$ se, e somente se, $x$ é q-positivo.

(ii) $x=x^{-}$se, e somente se, $x=-|x|$ se, e somente se, $x$ é q-negativo. 
(iii) $(-x)^{+}=-\left(x^{-}\right) e(-x)^{-}=-\left(x^{+}\right)$.

(iv) $|x| \geq 0 \leq x^{+}, x^{-} \leq 0 \geq-x^{+},|-x|=|x| e|x| \geq x$.

(v) $|x+y| \leq|x|+|y|, \quad|| x|-| y|| \leq|x-y|$ (desigualdade triangular).

(vi) Se $x \leq y e-x \leq y$, então $|x| \leq y$.

(vii) $x^{+}=x\left(\frac{1+\Theta_{\hat{\dot{x}}}}{2}\right)$ e $x^{-}=x\left(\frac{1-\Theta_{\dot{\hat{x}}}}{2}\right)$.

(viii) Se $A=\left\{\varphi \in \mathcal{A}_{0}(\mathbb{K}) \mid \hat{x}(\varphi) \geq 0\right\}$ então $x^{+}=x \mathcal{X}_{A}$ e $x^{-}=x \mathcal{X}_{A^{c}}$.

Prova. (i) $x=x^{+} \Leftrightarrow \frac{x-|x|}{2}=0 \Leftrightarrow x-|x|=0 \Leftrightarrow x=|x| \geq 0 \Leftrightarrow x$ é q-positivo.

(ii) $x=x^{-} \Leftrightarrow \frac{x+|x|}{2}=0 \Leftrightarrow x+|x|=0 \Leftrightarrow x=-|x| \leq 0 \Leftrightarrow x$ é q-negativo.

(iii) $(-x)^{+}=\frac{-x+|x|}{2}=-\frac{x-|x|}{2}=-x^{-}$e $(-x)^{-}=\frac{-x-|x|}{2}=-\frac{x+|x|}{2}=-x^{+}$.

(vi) Se $x \leq y$ e $-x \leq y$, então $-y \leq x \leq y$ e, portanto, $|x| \leq y$.

(vii) $x\left(\frac{1+\Theta_{\hat{x}}}{2}\right)=\frac{x+x \Theta_{\hat{x}}}{2}=\frac{x+|x|}{2}=x^{+}$e $x\left(\frac{1-\Theta_{\hat{x}}}{2}\right)=\frac{x-x \Theta_{\hat{x}}}{2}=\frac{x-|x|}{2}=x^{-}$.

(viii) Se $\varphi \in A$, então $\hat{x} \hat{\mathcal{X}}_{A}(\varphi)=\hat{x}(\varphi) \geq 0$, portanto, $x^{+}=x \mathcal{X}_{A}$. Agora, se $\varphi \in A^{c}$, então $\hat{x} \hat{\mathcal{X}}_{A}(\varphi)=\hat{x}(\varphi)<0$ e, portanto, $x^{-}=x \mathcal{X}_{A^{c}}$.

Observação 2.1.9. Note que se $z \in \overline{\mathbb{C}}_{f}$ então $|z| \in \overline{\mathbb{R}}_{f},|z| \geq 0$ e, portanto, podemos aplicar a Proposição 2.1 .8 onde for necessário. Em particular, a desigualdade triangular vale neste contexto.

Proposição 2.1.10 (Convexidade de ideais). Seja $\mathfrak{J}$ um ideal de $\overline{\mathbb{K}}_{f}$ e $x, y \in \overline{\mathbb{K}}_{f}$. Então:

(i) $x \in \mathfrak{J}$ se, e somente se, $|x| \in \mathfrak{J}$;

(ii) se $x \in \mathfrak{J}$ e $|y| \leq|x|$, então $y \in \mathfrak{J}$.

(iii) se $\mathbb{K}=\mathbb{R} e 0 \leq y \leq x$, então $y \in \mathfrak{J}$.

Prova. (i) Se $x \in \mathfrak{J}$, então $|x|=x \Theta_{\dot{x}} \in \mathfrak{J}$, pois $\mathfrak{J}$ é um ideal. Reciprocamente, se $|x| \in \mathfrak{J}$, então $|x|=x \Theta_{\hat{x}} \in \mathfrak{J}$. Agora, sendo $\Theta_{\hat{x}} \in \operatorname{Inv}\left(\overline{\mathbb{K}}_{f}\right)$ segue que $x=|x| \Theta_{\hat{x}}^{-1} \in \mathfrak{J}$, i.e., $x \in \mathfrak{J}$.

(ii) Se $|y|=|x|$, então por $(i)|x| \in \mathfrak{J}$, pois $x \in \mathfrak{J}$ e, portanto, $|y| \in \mathfrak{J}$ e pela recíproca de (i) segue que $y \in \mathfrak{J}$. Agora, se $|y|<|x|$, então $|x| \neq 0$, pois se $|x|=0$, então $|y|<0$ 
o que é um absurdo, pois $|y| \geq 0$. Seja $u=\frac{|y|}{|x|}$, então $|y|=u|x| \in \mathfrak{J}$, pois por $(i)|x| \in \mathfrak{J}$ $(x \in \mathfrak{J})$ e $\mathfrak{J}$ é um ideal de $\overline{\mathbb{K}}_{f}$. E mais uma vez pela recíproca de $(i)$, temos que $y \in \mathfrak{J}$.

(iii) Segue de (ii) e a Proposição 2.1.8.

Observação 2.1.11. Se $z \in \overline{\mathbb{C}}_{f}$, então é óbvio que podemos escrever $z=x+i y$, com $x, y \in \overline{\mathbb{R}}_{f}$, onde $i$ é classe da função constante $\sqrt{-1}$. Defina $\Re(z)=x$ e $\Im(z)=y$, as partes real e imaginária de $z$, respectivamente. Naturalmente, se $\hat{z}=\hat{x}+\sqrt{-1} \hat{y}$ é um representante de $z$, então $\hat{x}$ e $\hat{y}$ são representantes, respectivamente, de $x$ e y. É também, claro que $\overline{\mathbb{C}}_{f}$ é um $\overline{\mathbb{R}}_{f}$-módulo e que as aplicações $\Re, \Im: \overline{\mathbb{C}}_{f} \rightarrow \overline{\mathbb{R}}_{f}$ são $\overline{\mathbb{R}}_{f}$-epimorfismos. Portanto, se $\mathfrak{J} \triangleleft \overline{\mathbb{C}}_{f}$ é um ideal, então sua imagem por estes epimorfismos são ideais de $\overline{\mathbb{R}}_{f}$ e é um exercício trivial ver que eles coincidem; denotaremos por $\mathfrak{J}_{r}$ e chamamos a parte real do ideal $\mathfrak{J}$. Pela Proposição 2.1.10 segue que $\mathfrak{J}_{r} \subset \mathfrak{J}$.

Note que a involução

$$
C: \mathbb{C} \rightarrow \mathbb{C}
$$

definida por

$$
C(z)=\bar{z}
$$

se estende a uma involução $\overline{\mathbb{C}}_{f} \rightarrow \overline{\mathbb{C}}_{f}$ de $\overline{\mathbb{C}}_{f}$. Chamaremos esta involução de conjugação. O seguinte resultado é óbvio.

Lema 2.1.12. Seja $\mathfrak{J} \triangleleft \overline{\mathbb{C}}_{f}$ um ideal de $\overline{\mathbb{C}}_{f}$. Então

(i) $\mathfrak{J}_{r} \subset \mathfrak{J}$.

(ii) $\mathfrak{J}=\mathfrak{J}_{r}+i \mathfrak{J}_{r}$ e $\mathfrak{J}$ é invariante por conjugação.

(iii) $\mathfrak{J}_{r}=\mathfrak{J} \cap \overline{\mathbb{R}}_{f}$

Corolário 2.1.13. Seja $\mathcal{F} \in P_{*}\left(\mathcal{S}_{f}\right)$. Então:

(i) $g_{f_{r}}(\mathcal{F})$ é a parte real de $g_{f}(\mathcal{F})$ (ver a Definição 1.3.6).

(ii) Se $z \in \overline{\mathbb{C}}_{f}$, então $z \in g_{f}(\mathcal{F})$ se, e somente se, $|z| \in g_{f_{r}}(\mathcal{F})$.

Lema 2.1.14. Seja $\mathcal{F} \in P_{*}\left(\mathcal{S}_{f}\right)$ e $x, y \in \overline{\mathbb{R}}_{f}$. Então valem as seguintes afirmações:

(i) $S e(x-y) \in g_{f_{r}}(\mathcal{F})$ e $x^{-} \in g_{f_{r}}(\mathcal{F})$, então $y^{-} \in g_{f_{r}}(\mathcal{F})$. 
(ii) A parte $x^{+}$ou $x^{-}$de $x$ está em $g_{f_{r}}(\mathcal{F})$.

Prova. (i) Temos pela Proposição 2.1.8 item $(v)$ que

$$
\begin{aligned}
\left\|x^{-}-y^{-}\right\| & =\left\|\frac{x-|x|}{2}-\frac{y-|y|}{2}\right\| \\
& =\frac{1}{2}|x-| x|-y+| y|| \\
& \leq \frac{1}{2}(|x-y|+|| y|-| x||) \\
& \leq|x-y|,
\end{aligned}
$$

isto é, $\left\|x^{-}-y^{-}\right\| \leq|x-y|$. Como $(x-y) \in g_{f_{r}}(\mathcal{F})$ segue pela Proposição 2.1.10 item (ii) que $\left|x^{-}-y^{-}\right| \in g_{f_{r}}(\mathcal{F})$ e pela recíproca de 2.1.10 item $(i)$ temos que $x^{-}-y^{-} \in g_{f_{r}}(\mathcal{F})$ e como $x^{-} \in g_{f_{r}}(\mathcal{F})$ segue que $y^{-} \in g_{f_{r}}(\mathcal{F})$.

(ii) Podemos supor aqui que $x$ é q-positivo e não q-negativo e, portanto, $x$ tem um representante $\hat{x}$ tal que $\theta_{\hat{x}} \notin\{ \pm 1\}$ o que significa que que se $A:=\left\{\varphi \in \mathcal{A}_{0}(\mathbb{K}) \mid \theta_{\hat{x}} \equiv 1\right\}$, então $A$ ou $A^{c}$ está em $\mathcal{F}$ e o resultado segue da Proposição 2.1.8 item (viiii).

Definição 2.1.15. Seja $\alpha \in \overline{\mathbb{R}}_{f} / g_{f_{r}}(\mathcal{F})$. Dizemos que $\alpha$ é não-negativo $\alpha \geq 0$, se $\alpha$ tem um representante $a \in \overline{\mathbb{R}}_{f}$ tal que a parte negativa $a^{-} \in g_{f_{r}}(\mathcal{F})$. E isto nos dá uma relação de ordem no sentido usual.

O Lema 2.1.14 nos mostra que a Definição 2.1.15 é intrínseca, i.e., independe do representantes. O seguinte lema é fácil mostrar e muito conhecido:

Lema 2.1.16. Seja $(A, \leq)$ um anel comutativo com unidade parcialmente ordenado $e$ $a, b \in A$. Então:

(i) $(A, \leq)$ é totalmente ordenado se, e somente se, $a \geq 0$ ou $-a \geq 0$.

(ii) Se $\geq$ é uma relação de ordem total sobre $A$, então o nil-radical de $A$ é zero, isto é, $\mathcal{N}(A)=0$ e se $a, b \geq 0 \Rightarrow a b \geq 0$, então $A$ é um domínio integral.

Teorema 2.1.17. Seja $\mathcal{F} \in P_{*}\left(\mathcal{S}_{f}\right)$. Então $\left(\overline{\mathbb{R}}_{f} / g_{f_{r}}(\mathcal{F}), \leq\right)$ é um anel totalmente ordenado.

Prova. Inicialmente vamos mostrar que $\left(\mathbb{R}_{f} / g_{f_{r}}(\mathcal{F}), \leq\right)$ é um anel parcialmente ordenado. As propriedades reflexiva e anti-simétrica é facilmente provadas usando a Proposição 
2.1.8. Então resta mostrarmos que vale a propriedade transitiva. De fato, sejam $\alpha, \beta, \gamma \in$ $\left(\overline{\mathbb{R}}_{f} / g_{f_{r}}(\mathcal{F})\right)$ com representantes $a, b, c \in \overline{\mathbb{R}}_{f}$. Então se $\alpha \leq \beta$ e $\beta \leq \gamma$, queremos mostrar que $\alpha \leq \gamma$. Mas para isto precisamos mostrar que $(\gamma-\beta) \geq 0$, isto é, $(\gamma-\alpha)$ tem um representante $(c-a) \in \overline{\mathbb{R}}_{f}$ tal que $(c-a)^{-} \in g_{f_{r}}(\mathcal{F})$ (Definição 2.1.15). Ora, se $\alpha \leq \beta$ e $\beta \leq \gamma$, então $(\beta-\alpha) \geq 0$ e $(\gamma-\beta) \geq 0$, isto é, são ambos n-negativos e, pela Definição 2.1.15 os representantes $(b-a),(c-b) \in \overline{\mathbb{R}}_{f}$ de $(\beta-\alpha)$ e $(\gamma-\beta)$, respectivamente são tais que $(b-a)^{-},(c-b)^{-} \in g_{f_{r}}(\mathcal{F})$. Agora, pela desigualdade triangular, temos que

$$
\begin{aligned}
(c-a)^{-} & =\frac{1}{2}((c-a)-|c-a|) \\
& =\frac{1}{2}((c-b)+(b-a)-|(c-b)+(b-a)| \\
& \geq \frac{1}{2}\left((c-b)^{-}+(b-a)^{-}\right) \in g_{f_{r}}(\mathcal{F}) .
\end{aligned}
$$

$\mathrm{E}$, portanto, vale a propriedade transitiva. Vamos agora verificar se vale o lado direito de (i) do Lema 2.1.16. Para isto seja $\alpha \in\left(\overline{\mathbb{R}}_{f} / g_{f_{r}}(\mathcal{F}), \leq\right)$ e seja $a \in \overline{\mathbb{R}}_{f}$ um representante. Do Lema 2.1.14-(ii), temos que $a^{+}$ou $a^{-}$está em $g_{f_{r}}(\mathcal{F})$ e o resultado segue pela Proposição 2.1.8-(iii). E o Lema 2.1.16 nos da a assertiva.

\subsection{Outras propriedades algébricas de $\overline{\mathbb{K}}_{f}$}

Nesta seção damos continuidade aos fatos algébricos estudados no Capítulo 1, onde os ideais maximais de $\overline{\mathbb{K}}_{f}$ foram descrito completamente pelo Teorema 1.3.21. Agora nosso objetivo é descrever completamente os ideais primos de $\overline{\mathbb{K}}_{f}$ e mostrar que este anel não é Von Neumann regular.

Se $A$ é um anel comutativo com unidade, denotaremos por $\mathcal{B}(A)$ o conjunto dos idempotentes de $A$. Nosso primeiro resultado descreve completamente o conjunto dos idempotentes de $\overline{\mathbb{K}}_{f}$.

Teorema 2.2.1. Se e $\in \overline{\mathbb{K}}_{f}$ é um idempotente não-trivial, então existe $S \in \mathcal{S}_{f}$ tal que $e=\mathcal{X}_{S}$. Em particular, temos que $\mathcal{B}\left(\overline{\mathbb{K}}_{f}\right)$ é um subconjunto discreto de $\overline{\mathbb{K}}_{f}$.

Prova. Seja $\hat{e}=e(\varphi)$ um representante qualquer de $e$. Então como $e^{2}=e$ segue que $e(1-e)=0$, isto é, $\hat{e}(1-\hat{e}) \in \mathcal{N}_{f}(\mathbb{K})$ e, portanto, existem $p \in \mathbb{N}, \gamma \in \Gamma$ tal que para todo 
$q \geq p$ e para toda $\varphi \in \mathcal{A}_{q}(\mathbb{K})$ existem $C=C(\varphi)>0, \eta=\eta(\varphi)>0$ tal que

$$
\left|\hat{e}\left(\varphi_{\varepsilon}\right)\left(1-\hat{e}\left(\varphi_{\varepsilon}\right)\right)\right| \leq C \varepsilon^{\gamma(q)-p}, \forall 0<\varepsilon<\eta .
$$

Sejam $S=\left(N_{a}(\hat{e})\right)^{c}=\left\{\varphi \in \mathcal{A}_{0}(\mathbb{K}) \| e(\varphi) \mid \geq \dot{\alpha}_{a}(\varphi)\right\}$ e $\hat{u}=\hat{e}-\hat{\mathcal{X}}_{S}$. Então vamos mostrar que $\hat{u} \in \mathcal{N}_{f}(\mathbb{K})$. De fato, se $\hat{u}=\hat{e}-\hat{\mathcal{X}}_{S}$, então para todo $0<\varepsilon<\eta$, temos

$$
\left|\hat{u}\left(\varphi_{\varepsilon}\right)\right|= \begin{cases}\left|1-\hat{e}\left(\varphi_{\varepsilon}\right)\right|, & \text { se } \varphi_{\varepsilon} \in S \\ \left|\hat{e}\left(\varphi_{\varepsilon}\right)\right|, & \text { se } \varphi_{\varepsilon} \in S^{c} .\end{cases}
$$

Ora, se $\varphi_{\varepsilon} \in S$, então $\left|\hat{e}\left(\varphi_{\varepsilon}\right)\right| \geq \dot{\alpha}_{a}\left(\varphi_{\varepsilon}\right)=(i(\varphi))^{a} \varepsilon^{a}$ e, portanto,

$$
\frac{1}{\left|\hat{e}\left(\varphi_{\varepsilon}\right)\right|} \leq(i(\varphi))^{-a} \varepsilon^{-a}, \forall 0<\varepsilon<\eta,
$$

observando que $\left|\hat{e}\left(\varphi_{\varepsilon}\right)\right| \neq 0$, pois $e$ é um idempotente não-trivial. Assim, temos

$$
\begin{aligned}
\left|\hat{u}\left(\varphi_{\varepsilon}\right)\right| & =\left|1-\hat{e}\left(\varphi_{\varepsilon}\right)\right| \\
& =\frac{1}{\left|\hat{e}\left(\varphi_{\varepsilon}\right)\right|}\left|\hat{e}\left(\varphi_{\varepsilon}\right)\right|\left|1-\hat{e}\left(\varphi_{\varepsilon}\right)\right| \\
& \left.\leq \frac{1}{\left|\hat{e}\left(\varphi_{\varepsilon}\right)\right|}\left|\hat{e}\left(\varphi_{\varepsilon}\right)\left(1-\hat{e}\left(\varphi_{\varepsilon}\right)\right)\right| \quad \text { (por }(2.1) \text { e }(2.2), \text { temos }\right) \\
& \leq\left((i(\varphi))^{-a} \varepsilon^{-a}\right)\left(C \varepsilon^{\gamma(q)-p}\right), \forall 0<\varepsilon<\eta \\
& =C(i(\varphi))^{-a} \varepsilon^{\gamma(q)-(p+a)}, \forall 0<\varepsilon<\eta .
\end{aligned}
$$

Portanto,

$$
\left|\hat{u}\left(\varphi_{\varepsilon}\right)\right| \leq K \varepsilon^{\gamma(q)-P}, \forall 0<\varepsilon<\eta,
$$

onde $K=C(i(\varphi))^{-a}>0$ e $P=p+a$.

Agora, se $\varphi_{\varepsilon} \in S^{c}=N_{a}(\hat{e}), \forall 0<\varepsilon<\eta$, então

$$
\left|\hat{e}\left(\varphi_{\varepsilon}\right)\right|<\dot{\alpha}_{a}\left(\varphi_{\varepsilon}\right)=(i(\varphi))^{a} \varepsilon^{a} \Rightarrow\left|\hat{e}\left(\varphi_{\varepsilon}\right)\right|<(i(\varphi))^{a} \varepsilon^{a}, \forall 0<\varepsilon<\eta .
$$

Mas neste caso, temos que $\left|\hat{u}\left(\varphi_{\varepsilon}\right)\right|=\left|\hat{e}\left(\varphi_{\varepsilon}\right)\right|$ e por (2.4) segue que

$$
\left|\hat{u}\left(\varphi_{\varepsilon}\right)\right| \leq(i(\varphi))^{a} \varepsilon^{a}, \forall 0<\varepsilon<\eta .
$$

Logo, por (2.3) e (2.5) temos que $\hat{u} \in \mathcal{N}_{f}(\mathbb{K})$ e, portanto, $\hat{e}=\hat{\mathcal{X}}_{S}$, i.e., $e=\mathcal{X}_{S}$. Em particular, temos que $\mathcal{B}\left(\overline{\mathbb{K}}_{f}\right)$ é um subconjunto discreto de $\overline{\mathbb{K}}_{f}$.

Definição 2.2.2. Um anel com unidade é dito Von Neumann regular se todos os seus ideais principais são gerados por um idempotente. 
Para o que queremos fazer basta a seguinte:

Proposição 2.2.3. Seja $A$ um anel comutativo com unidade e seja $\mathcal{N}(A)$ seu nil-radical. Então todo ideal primo de $A$ é maximal se, e somente se, $A / \mathcal{N}(A)$ é Von Neumann regular.

Lema 2.2.4. Seja $\gamma: \mathcal{A}_{0}(\mathbb{K}) \rightarrow \mathbb{R} \cup\{+\infty\}$ tal que

$$
\gamma(\varphi)= \begin{cases}+\infty, & \text { se }(i(\varphi))^{-1} \notin \mathbb{N} \\ p, & \text { se }(i(\varphi))^{-1} \in \mathbb{N}\end{cases}
$$

onde $p$ é o menor primo que divide $(i(\varphi))^{-1}$. Seja $x \in \overline{\mathbb{K}}_{f}$ tal que $\hat{x}(\varphi)=\hat{\dot{\alpha}}_{\gamma(\varphi)}(\varphi)$ é um representante. Então o ideal gerado por $x$ não é um ideal idempotente.

Prova. Suponhamos que $\mathfrak{J}=x \overline{\mathbb{K}}_{f}$ é um ideal idempotente. Então pelo Teorema 2.2.1 existe $S \in \mathcal{S}_{f}$ tal que $\mathfrak{J}=\mathcal{X}_{S} \overline{\mathbb{K}}_{f}$. Pela definição de $\gamma$ temos dois casos a analisar, a saber:

(a) $\gamma(S)$ é finito;

(b) $\gamma(S)$ é infinito.

(a) Sejam $\gamma(S)$ finito, $\sigma:=\max \{z \mid z \in \mathbb{R} \cap \gamma(S)\}$ e fixemos um número primo $p>\sigma$. Seja também $\varepsilon_{n}:=p^{-n}$. Então $\varepsilon_{n} \underset{n \rightarrow \infty}{\longrightarrow} 0$ e $\gamma\left(\varphi_{\varepsilon_{n}}\right)=p \notin \gamma(S), \forall n \in \mathbb{N}$ e, portanto, $\varphi_{\varepsilon_{n}} \notin S$, isto é, $\varphi_{\varepsilon_{n}} \in S^{c}$. Agora,

$$
\hat{x}\left(\varphi_{\varepsilon_{n}}\right)=\hat{\dot{\alpha}}_{\gamma\left(\varphi_{\varepsilon_{n}}\right)}\left(\varphi_{\varepsilon_{n}}\right)=\hat{\dot{\alpha}}_{p}\left(\varphi_{\varepsilon_{n}}\right)=(i(\varphi))^{p} \varepsilon_{n}^{p}>0, \forall n \in \mathbb{N}
$$

e, portanto, $x \mathcal{X}_{S^{c}} \neq 0$. Suponhamos, que $x \in \mathfrak{J}=\mathcal{X}_{S} \overline{\mathbb{K}}_{f}$, então $x=y \mathcal{X}_{S}$ para algum $y \in \overline{\mathbb{K}}_{f}$. Daí, temos que $0=\left(y \mathcal{X}_{S}\right) \mathcal{X}_{S^{c}}=x \mathcal{X}_{S^{c}} \neq 0$ o que é uma contradição.

(b) Agora, se $\gamma(S)$ é infinito, então existe uma seqüência $\left\{\varepsilon_{n}\right\} \subset\left\{\varepsilon \mid \varphi_{\varepsilon} \in S\right\}$ que converge a zero quando $n \rightarrow \infty$ tal que $\left\{\gamma\left(\varphi_{\varepsilon_{n}}\right)\right\} \subset \mathbb{N}$ é uma seqüência estritamente crescente e divergente. Suponhamos que $\mathcal{X}_{S}=y x$. Então

$$
\hat{\mathcal{X}}_{S}\left(\varphi_{\varepsilon}\right)-\hat{y}\left(\varphi_{\varepsilon}\right) \hat{x}\left(\varphi_{\varepsilon}\right)=1-\hat{y}\left(\varphi_{\varepsilon}\right) \hat{x}\left(\varphi_{\varepsilon}\right) \underset{n \rightarrow \infty}{\longrightarrow} 0
$$

Mas visto que $\left\{\gamma\left(\varphi_{\varepsilon_{n}}\right)\right\}$ é uma seqüência crescente divergente segue facilmente que $y$ não pode ser uma função moderada, isto é, $y \notin \mathcal{E}_{f}^{M}(\mathbb{K})$ o que é uma contradição. 
Das duas contradições encontradas nos casos $(a)$ e $(b)$ segue o resultado.

Com o que desenvolvemos até este ponto podemos afirmar que $\overline{\mathbb{K}}_{f}$ não é Von Neumann regular segundo a Definição 2.2.2, mais precisamente, temos:

Teorema 2.2.5. $\overline{\mathbb{K}}_{f}$ não é Von Neumann regular. Em particular, existe $\mathcal{F} \in P_{*}\left(\mathcal{S}_{f}\right)$ tal que $g_{f}(\mathcal{F})$ não é fechado e $\overline{\mathbb{K}}_{f}$ tem um ideal primo que não é um ideal maximal.

Prova. Sabemos pelo Teorema 1.3.20 do Capítulo 1 que o nil-radical de $\overline{\mathbb{K}}_{f}$ é nulo, isto é, $\mathcal{N}\left(\overline{\mathbb{K}}_{f}\right)=\{0\}$. Portanto, pela Proposição 2.2.3 e o Lema 2.2.4 segue que $\overline{\mathbb{K}}_{f}$ não é Von Neumann regular.

Teorema 2.2.6. Para todo $\mathcal{F} \in P_{*}\left(\mathcal{S}_{f}\right), g_{f}(\mathcal{F})$ é um ideal primo.

Prova. Inicialmente, suponhamos que $\mathbb{K}=\mathbb{R}$. Precisamos mostrar que a condição (ii) do Lema 2.1.16 se verifica. Assim, sejam $a, b \in \overline{\mathbb{R}}_{f}$ tal que $a^{-}, b^{-} \in g_{f}(\mathcal{F})$. Então

$$
\begin{aligned}
(a b)^{-} & =\frac{1}{2}(a b-|a||b|) \\
& =\frac{1}{2}\left[\left(a^{+}+a^{-}\right)\left(b^{+}+b^{-}\right)-\left(a^{+}-a^{-}\right)\left(b^{+}-b^{-}\right)\right] \\
& =a^{+} b^{-}+a^{-} b^{+}
\end{aligned}
$$

e, portanto, $(a b)^{-} \in g_{f}(\mathcal{F})$, pois $a^{+} b^{-}+a^{-} b^{+} \in g_{f}(\mathcal{F})$. Isto significa que o cone positivo é invariante por multiplicação. Agora, seja $a \in \overline{\mathbb{R}}_{f}$ tal que $a^{2} \in g_{f}(\mathcal{F})$. Da definição do ideal $g_{f}(\mathcal{F})$ (ver Capítulo 1 ), temos que existe $A \in \mathcal{F}$ tal que $a^{2}=a^{2} \mathcal{X}_{A}$. Portanto, $a^{2} \mathcal{X}_{A^{c}}=0$ e assim $a \mathcal{X}_{A^{c}} \in \mathcal{N}\left(\overline{\mathbb{R}}_{f}\right)=0$. Logo, $a=a \mathcal{X}_{A}+a \mathcal{X}_{A^{c}}=a \mathcal{X}_{A} \in g_{f}(\mathcal{F})$. Assim, estamos feito neste caso. Para completarmos a prova consideremos o caso em que $\mathbb{K}=\mathbb{C}$. Sejam $x, y \in g_{f}(\mathcal{F})$ tal que $x y \in g_{f}(\mathcal{F})$. Então $|x y|=|x||y| \in \overline{\mathbb{R}}_{f} \cap g_{f}(\mathcal{F})=g_{f_{r}}(\mathcal{F})$. Pelo primeiro caso $|x|$ ou $|y|$ está em $g_{f_{r}}(\mathcal{F})$ e pela convexidade de ideais segue o resultado.

Corolário 2.2.7. $\left\{g_{f_{r}}(\mathcal{F}) \mid \mathcal{F} \in P_{*}\left(\mathcal{S}_{f}\right)\right\}$ é o conjunto dos ideais primos de $\overline{\mathbb{R}}_{f} e$

$$
\left\{g_{f}(\mathcal{F}) \mid \mathcal{F} \in P_{*}\left(\mathcal{S}_{f}\right)\right\}
$$

é o conjunto dos ideais primos de $\overline{\mathbb{C}}_{f}$. 


\section{A topologia cortante sobre a álgebra das funções generalizadas plenas de Colombeau}

Neste capítulo vamos construir uma topologia para a álgebra das funções generalizadas plenas de Colombeau $\mathcal{G}_{f}(\Omega)$, semelhante àquela construída por D. Scarpalézos, para a álgebra das funções generalizadas simplificadas de Colombeau $\mathcal{G}(\Omega)$. (Ver [15, 16].) Esta topologia será chamada a topologia cortante plena e denotaremos por $\tau_{\Omega f}$. Veremos que esta topologia é Hausdorff e estudamos alguns resultados sobre convergência em $\left(\mathcal{G}_{f}(\Omega), \tau_{\Omega f}\right)$, dentre eles destacamos que $\mathcal{G}_{f_{c}}(\Omega)$ é denso em $\mathcal{G}_{f}(\Omega)$. (Ver Corolário 3.3.9.) Ainda neste capítulo, definimos uma topologia sobre $\mathcal{G}_{f}(\bar{\Omega})$, onde $\Omega \subset \mathbb{R}^{n}$ é um domínio limitado e $\bar{\Omega}$ é o fecho topológico de $\Omega$. (Ver [4].) Mostraremos que com esta topologia, denotada por $\tau_{\bar{\Omega} b}, \mathcal{G}_{f}(\bar{\Omega})$ é completa (Teorema 3.4.13). (Ver [4].) Como conseqüência do Teorema 3.4.13, temos o Corolário 3.4.14. Veremos que os resultados de densidade e completude, descritos acima, serão a mola mestra para os estudos que realizaremos no Capítulo 4, onde pretendemos generalizar os resultados de existência e unicidade de solução para um problema de valor inicial e de fronteira, abreviadamente PVIF, do tipo parabólico . (Ver [10].) 
A topologia cortante sobre a álgebra das funções generalizadas plenas de 58

Colombeau

\subsection{A álgebra das funções generalizadas}

Seja $\Omega$ um subconjunto aberto não-vazio de $\mathbb{R}^{n}$. Denote por $K \subset \subset \Omega$ para significar que $K$ é um subconjunto compacto de $\Omega$. Para uma função $f: \Omega \rightarrow \mathbb{K}$ e $\emptyset \neq \Omega^{\prime} \subset \Omega$ definimos

$$
\|f\|_{\Omega^{\prime}}:=\sup _{x \in \Omega^{\prime}}|f(x)|
$$

Uma exaustão para um subconjunto aberto $\Omega \subset \mathbb{R}^{n}$ é uma seqüência $\left\{\Omega_{m}\right\}_{m \in \mathbb{N}}$ de subconjuntos abertos não-vazios de $\Omega$ tal que

$$
\Omega=\bigcup_{m \in \mathbb{N}} \Omega_{m} \quad \text { e } \quad \bar{\Omega}_{m} \subset \subset \Omega_{m+1}, \forall m \in \mathbb{N}
$$

No caso particular em que $\Omega^{\prime}=\Omega_{m}(\forall m \in \mathbb{N})$ escrevemos em (3.1) simplesmente $\|f\|_{m}$ ao invés de $\|f\|_{\Omega_{m}}$, isto é,

$$
\|f\|_{m}=\sup _{x \in \Omega_{m}}|f(x)|
$$

\subsubsection{Funções generalizadas sobre um conjunto aberto}

Seja

$$
\mathcal{E}_{f}(\Omega):=\left\{u: \mathcal{A}_{0}(\mathbb{K}) \times \Omega \rightarrow \mathbb{K} \mid u(\varphi, \cdot) \in \mathrm{C}^{\infty}(\Omega), \forall \varphi \in \mathcal{A}_{0}(\mathbb{K})\right\}
$$

o anel (operações pontuais) de todas as funções $u: \mathcal{A}_{0}(\mathbb{K}) \times \Omega \rightarrow \mathbb{K}$ tal que

$$
u(\varphi, \cdot) \in \mathrm{C}^{\infty}(\Omega), \forall \varphi \in \mathcal{A}_{0}(\mathbb{K})
$$

Se $\alpha \in \mathbb{N}^{n}$ e $x \in \Omega$ definimos

$$
\partial^{\alpha} u(\varphi, x):=\partial^{\alpha} u(\varphi, \cdot)(x)
$$

Seja

$$
\begin{gathered}
\mathcal{E}_{f}^{M}(\Omega):=\left\{u \in \mathcal{E}_{f}(\Omega) \mid \forall K \subset \subset \Omega, \forall \alpha \in \mathbb{N}^{n}, \exists p \in \mathbb{N} \text { tal que } \forall \varphi \in \mathcal{A}_{p}(\mathbb{K})\right. \\
\exists C=C(\varphi)>0, \exists \eta=\eta(\varphi)>0 \text { tal que } \\
\left.\sup _{x \in K}\left|\partial^{\alpha} u\left(\varphi_{\varepsilon}, x\right)\right| \leq C \varepsilon^{-p}, \forall 0<\varepsilon<\eta\right\}
\end{gathered}
$$


o subanel de $\mathcal{E}_{f}(\Omega)$ das funções moderadas e o seu ideal

$$
\begin{aligned}
\mathcal{N}_{f}(\Omega):=\left\{u \in \mathcal{E}_{f}^{M}(\Omega) \mid\right. & \forall K \subset \subset \Omega, \forall \alpha \in \mathbb{N}^{n}, \exists p \in \mathbb{N}, \exists \gamma \in \Gamma \text { tal que } \\
& \forall q \geq p, \forall \varphi \in \mathcal{A}_{p}(\mathbb{K}), \exists C=C(\varphi)>0, \exists \eta=\eta(\varphi)>0 \\
& \text { tal que } \left.\sup _{x \in K}\left|\partial^{\alpha} u\left(\varphi_{\varepsilon}, x\right)\right| \leq C \varepsilon^{\gamma(q)-p}, \forall 0<\varepsilon<\eta\right\},
\end{aligned}
$$

onde $\Gamma$ é o conjunto das seqüências estritamente crescente, divergente. Então definimos a álgebra das funções generalizadas plenas de Colombeau $\mathcal{G}_{f}(\Omega)$ como sendo o anel quociente $\mathcal{E}_{f}^{M}(\Omega) / \mathcal{N}_{f}(\Omega)$, isto é,

$$
\mathcal{G}_{f}(\Omega):=\mathcal{E}_{f}^{M}(\Omega) / \mathcal{N}_{f}(\Omega)
$$

$\mathrm{Na}$ linguagem da exaustão é conveniente dá a seguinte caracterização bem conhecida, ver por exemplo, Aragona, Fernandez e Juriaans [3], dos elementos de $\mathcal{E}_{f}^{M}(\Omega)$ e $\mathcal{N}_{f}(\Omega)$.

Proposição 3.1.1. (i) $u \in \mathcal{E}_{f}^{M}(\Omega)$ se, e somente se, $\forall(m, p) \in \mathbb{N}^{2}, \exists N \in \mathbb{N}, \exists \sigma \in \mathbb{R}$ tal que $\forall \varphi \in \mathcal{A}_{N}(\mathbb{K})$ temos $\left\|\partial^{\beta} u\left(\varphi_{\varepsilon}, \cdot\right)\right\|_{m}=o\left(\varepsilon^{\sigma}\right)$, se $\varepsilon \downarrow 0, \forall|\beta| \leq p ;$

(ii) $u \in \mathcal{N}_{f}(\Omega)$ se, e somente se, $\forall(m, p) \in \mathbb{N}^{2} \forall \sigma \in \mathbb{R}, \exists N \in \mathbb{N}$ tal que $\forall \varphi \in \mathcal{A}_{N}(\mathbb{K})$ temos $\left\|\partial^{\beta} u\left(\varphi_{\varepsilon}, \cdot\right)\right\|_{m}=o\left(\varepsilon^{\sigma}\right)$, se $\varepsilon \downarrow 0, \forall|\beta| \leq p$.

Pela Proposição 3.1.1, temos o seguinte:

Corolário 3.1.2. (i) Se $u \in \mathcal{E}_{f}^{M}(\Omega)$, então para todo $(m, p) \in \mathbb{N}^{2}$ existem $N \in \mathbb{N}, r \in$ $\mathbb{R}$ tal que para toda $\varphi \in \mathcal{A}_{N}(\mathbb{K})$ existe $\eta=\eta(\varphi) \in I$ tal que

$$
\left\|\partial^{\beta} u\left(\varphi_{\varepsilon}, \cdot\right)\right\|_{m} \leq \dot{\alpha}_{r}\left(\varphi_{\varepsilon}\right), \forall \varepsilon \in I_{\eta}, \forall|\beta| \leq p
$$

(ii) Se $u \in \mathcal{N}_{f}(\Omega)$, então para todo $(m, p) \in \mathbb{N}^{2}$ e para todo $r \in \mathbb{R}$ existe $N \in \mathbb{N}$ tal que para toda $\varphi \in \mathcal{A}_{N}(\mathbb{K})$ existe $\eta=\eta(\varphi) \in I$ tal que

$$
\left\|\partial^{\beta} u(\varphi \varepsilon, \cdot)\right\|_{m} \leq \dot{\alpha}_{r}\left(\varphi_{\varepsilon}\right), \forall \varepsilon \in I_{\eta}, \forall|\beta| \leq p
$$

Prova. (i) Pela Proposição 3.1.1 $u \in \mathcal{E}_{f}^{M}(\Omega)$, se, e somente se, $\forall(m, p) \in \mathbb{N}^{2}, \exists N \in$ $\mathbb{N}, \exists r \in \mathbb{R}$ tal que $\forall \varphi \in \mathcal{A}_{N}(\mathbb{K})$ tem-se que $\left\|\partial^{\beta} u\left(\varphi_{\varepsilon}, \cdot\right)\right\|_{m}=o\left(\varepsilon^{r}\right)$, se $\varepsilon \downarrow 0, \forall|\beta| \leq p$. Agora, pela definição de ordem, se e, somente se,

$$
\lim _{\varepsilon \downarrow 0} \frac{\left\|\partial^{\beta} u\left(\varphi_{\varepsilon}, \cdot\right)\right\|_{m}}{\varepsilon^{r}}=0, \forall|\beta| \leq p
$$


Como $(i(\varphi))^{r}>0$ e não depende de $\varepsilon$ se, e somente se,

$$
\lim _{\varepsilon \downarrow 0} \frac{\left\|\partial^{\beta} u\left(\varphi_{\varepsilon}, \cdot\right)\right\|_{m}}{(i(\varphi))^{r} \varepsilon^{r}}=0, \forall|\beta| \leq p .
$$

Substituindo $(i(\varphi))^{r} \varepsilon^{r}=\dot{\alpha}_{r}\left(\varphi_{\varepsilon}\right)$ em (3.2) vemos que a expressão em (3.2) implica que

$$
\frac{\left\|\partial^{\beta} u\left(\varphi_{\varepsilon}, \cdot\right)\right\|_{m}}{\dot{\alpha}_{r}\left(\varphi_{\varepsilon}\right)} \leq 1, \forall|\beta| \leq p
$$

e para todo $\varepsilon$ suficientemente pequeno. Portanto, se $u \in \mathcal{E}_{f}^{M}(\Omega)$, então para todo $(m, p) \in$ $\mathbb{N}^{2}, \exists N \in \mathbb{N}, \exists r \in \mathbb{R}$ tal que $\forall \varphi \in \mathcal{A}_{N}(\mathbb{K}), \exists \eta=\eta(\varphi) \in I$ tal que

$$
\left\|\partial^{\beta} u\left(\varphi_{\varepsilon}, \cdot\right)\right\|_{m} \leq \dot{\alpha}_{r}\left(\varphi_{\varepsilon}\right), \forall \varepsilon \in I_{\eta}, \forall|\beta| \leq p .
$$

Para mostrar (ii) usamos o mesmo tipo de raciocínio que na parte $(i)$.

O lema seguinte, devido a Aragona, Fernandez e Juriaans [3], associa a cada elemento $u \in \mathcal{E}_{f}^{M}(\Omega)$ um elemento $u_{m}^{\beta} \in \mathcal{E}_{f}^{M}(\mathbb{R})$, onde $\beta \in \mathbb{N}^{n}$ e $m \in \mathbb{N}$, mais precisamente, temos:

Lema 3.1.3. Para todo $u \in \mathcal{E}_{f}^{M}(\Omega), \beta \in \mathbb{N}^{n}$ e $m \in \mathbb{N}$, temos:

(i) A função $u_{m}^{\beta}: \mathcal{A}_{0}(\mathbb{K}) \rightarrow \mathbb{R}_{+}$tal que $u_{m}^{\beta}(\varphi)=\left\|\partial^{\beta} u(\varphi, \cdot)\right\|_{m}$ é moderada (i.e., $u_{m}^{\beta} \in$ $\mathcal{E}_{f}^{M}(\mathbb{R}) e \operatorname{cl}\left[u_{m}^{\beta}\right] \in \overline{\mathbb{R}}_{f_{+}} ;$

(ii) se $v \in \mathcal{E}_{f}^{M}(\Omega)$ e $u-v \in \mathcal{N}_{f}(\Omega)$, então $u_{m}^{\beta}-v_{m}^{\beta} \in \mathcal{N}_{f}(\mathbb{R})$ e, portanto, $\operatorname{cl}\left[u_{m}^{\beta}\right]=\operatorname{cl}\left[v_{m}^{\beta}\right]$.

Prova. Ver [3].

\subsubsection{Funções generalizadas sobre a fronteira de um conjunto aberto}

Com o objetivo de tornar este texto um pouco mais completo, resolvemos incluir esta subseção de autoria do Aragona [1], mas é claro que vamos adaptar suas notações para as nossas e, os resultados serão indicados sem suas provas. Vamos supor que $\Omega$ é um subconjunto aberto de $\mathbb{R}^{n}$ com $n \geq 1$. Se $u \in \mathcal{E}_{f}^{M}(\bar{\Omega})$ (resp. $u \in \mathcal{N}_{f}(\bar{\Omega})$ ) e consideramos a restrição $\left.u\right|_{\mathcal{A}_{0}(\mathbb{K}) \times \partial \Omega}$, então a definição de $\mathcal{E}_{f}^{M}(\bar{\Omega})\left(\right.$ resp. $\left.\mathcal{N}_{f}(\bar{\Omega})\right)$ mostra que $\left.u\right|_{\mathcal{A}_{0}(\mathbb{K}) \times \partial \Omega}$ satisfaz a condição de moderação (resp. nulidade) que motiva a definição abaixo: 
Definição 3.1.4. (a). Uma função $u: \mathcal{A}_{0}(\mathbb{K}) \times \partial \Omega \rightarrow \mathbb{K}$ é dita 0-moderada se para cada $K \subset \subset \partial \Omega$ existe $N \in \mathbb{N}$ tal que para toda $\varphi \in \mathcal{A}_{N}(\mathbb{K})$ podemos encontrar $c>0 e$ $\eta \in I$ verificando

$$
\left|u\left(\varphi_{\varepsilon}, x\right)\right| \leq c \varepsilon^{-N}, \forall x \in K, \forall \varepsilon \in I_{\eta}
$$

(b) Uma função $u: \mathcal{A}_{0}(\mathbb{K}) \times \partial \Omega \rightarrow \mathbb{K}$ é dita 0 -nula se para cada $K \subset \subset \partial \Omega$ existe $\gamma \in \Gamma$ e $N \in \mathbb{N}$ tal que para todo $q \geq N$ e para toda $\varphi \in \mathcal{A}_{q}(\mathbb{K})$ podemos encontrar $c>0$ e $\eta \in I$ verificando

$$
\left|u\left(\varphi_{\varepsilon}, x\right)\right| \leq c \varepsilon^{\gamma(q)-N}, \forall x \in K, \forall \varepsilon \in I_{\eta} .
$$

Claramente o conjunto

$$
\begin{aligned}
\mathcal{E}_{f}^{M}(\partial \Omega) & =\mathcal{E}_{f}^{M}(\partial \Omega ; \mathbb{K}) \\
& :=\left\{u: \mathcal{A}_{0}(\mathbb{K}) \times \partial \Omega \rightarrow \mathbb{K} \mid u \text { é } 0 \text {-moderada e } u(\varphi, \cdot) \in \mathrm{C}(\partial \Omega ; \mathbb{K}), \forall \varphi \in \mathcal{A}_{0}(\mathbb{K})\right\}
\end{aligned}
$$

munida com as operações usuais ponto a ponto, é uma $\mathbb{K}$-álgebra e o conjunto

$$
\mathcal{N}_{f}(\partial \Omega)=\mathcal{N}_{f}(\partial \Omega ; \mathbb{K}):=\left\{u \in \mathcal{E}_{f}^{M}(\partial \Omega) \mid u \text { é } 0 \text {-nula }\right\}
$$

é um ideal de $\mathcal{E}_{f}^{M}(\partial \Omega)$. Portanto, a definição seguinte é significativa.

Definição 3.1.5. $\mathcal{G}_{f}^{b}(\partial \Omega):=\mathcal{E}_{f}^{M}(\partial \Omega) / \mathcal{N}_{f}(\partial \Omega)$ é a $\mathbb{K}$-álgebra das funções generalizadas sobre a fronteira $\partial \Omega$ do conjunto aberto $\Omega$. Denotamos por

$$
\pi_{\partial \Omega}=\pi_{\partial \Omega ; \mathbb{K}}: \mathcal{E}_{f}^{M}(\partial \Omega) \rightarrow \mathcal{G}_{f}^{b}(\partial \Omega)
$$

a aplicação quociente.

Aqui a notação $\mathcal{G}_{f}(X)$ é usada para a álgebra das funções generalizadas num conjunto quase-regular, i.e., em subconjuntos $X \subset \mathbb{R}^{n}$ do tipo $\Omega \cup F$, onde $\Omega$ é um subconjunto aberto não-vazio de $\mathbb{R}^{n}$ e $F \subset \partial \Omega$, de tal modo que, no caso $F=\emptyset$ temos as funções generalizadas usual definida sobre $\Omega$ (ver [1] pg-371). Na literatura, o símbolo $\mathcal{G}_{f}(X)$ foi usado para denotar uma álgebra de funções generalizadas sobre $X$, um subconjunto qualquer, não-vazio, de $\mathbb{R}^{n}$. Na definição acima usamos a notação $\mathcal{G}_{f}^{b}$, para nossa álgebra sobre $\partial \Omega$, para não confundir com esta notação anterior. 
Fixemos $f \in \mathcal{G}_{f}(\bar{\Omega})$ qualquer. Se $\hat{f}_{i} \in \mathcal{E}_{f}^{M}(\bar{\Omega}),(i=1,2)$ são dois representantes de $f$ então $\left.\hat{f}_{i}\right|_{\mathcal{A}_{0} \times \partial \Omega} \in \mathcal{E}_{f}^{M}(\partial \Omega)(i=1,2)$ e de $\hat{f}_{1}-\hat{f}_{2} \in \mathcal{N}_{f}$ segue que $\left.\left(\hat{f}_{1}-\hat{f}_{2}\right)\right|_{\mathcal{A}_{0} \times \partial \Omega} \in \mathcal{N}_{f}(\partial \Omega)$, portanto temos uma aplicação de restrição natural

$$
R_{\partial \Omega}^{\bar{\Omega}}: f \in \mathcal{G}_{f}(\bar{\Omega}) \mapsto \pi_{\partial \Omega}\left(\left.\hat{f}_{i}\right|_{\mathcal{A}_{0} \times \partial \Omega}\right) \in \mathcal{G}_{f}^{b}(\partial \Omega)
$$

onde $\hat{f}$ é um representante de $f$. Se não surgir nenhuma confusão, denotaremos $R_{\partial \Omega}^{\bar{\Omega}}(f)$ simplesmente por $\left.f\right|_{\partial \Omega}$.

Exemplo 3.1.6. (a) A aplicação $f \in \mathrm{C}(\partial \Omega) \mapsto \pi_{\partial \Omega}(\tilde{f}) \in \mathcal{G}_{f}^{b}(\partial \Omega)$, onde

$$
\tilde{f}(\varphi, x):=f(x), \forall(\varphi, x) \in \mathcal{A}_{0}(\mathbb{K}) \times \partial \Omega
$$

é um homomorfismo natural injetivo de $\mathbb{K}$-álgebras que identifica canonicamente $\mathrm{C}(\partial \Omega)$ com sua imagem em $\mathcal{G}_{f}^{b}(\partial \Omega)$ e permite escrever $\mathrm{C}(\partial \Omega) \subset \mathcal{G}_{f}^{b}(\partial \Omega)$.

(b) Se $V$ é um conjunto aberto contendo $\partial \Omega$ e $\hat{g} \in \mathcal{E}_{f}^{M}(V)$ é um representante de uma dada $g \in \mathcal{G}_{f}(V)$, então $\hat{h}:=\left.\hat{g}\right|_{\mathcal{A}_{0} \times \partial \Omega} \in \mathcal{E}_{f}^{M}(\partial \Omega)$ e sua imagem em $\mathcal{G}_{f}^{b}(\partial \Omega)$ é independente do representante $\hat{g}$ de $g$, o que fornece uma aplicação natural de restrição

$$
R_{V, \partial \Omega}: g \in \mathcal{G}_{f}(V) \mapsto \pi_{\partial \Omega}\left(\left.\hat{g}\right|_{\left.\mathcal{A}_{0}(\mathbb{K}) \times \partial \Omega\right)}\right) \in \mathcal{G}_{f}^{b}(\partial \Omega),
$$

onde $\hat{g}$ é um representante arbitrário de g. Se não surgir nenhuma confusão, denotaremos $R_{V, \partial \Omega}(g)$ simplesmente por $\left.g\right|_{\partial \Omega}$.

Surge então a seguinte questão: Dados quaisquer $f \in \mathcal{G}_{f}(\Omega)$ e $g \in \mathcal{G}_{f}^{b}(\partial \Omega)$, encontrar condições suficientes para a existência de $F \in \mathcal{G}_{f}(\bar{\Omega})$ tal que $\left.F\right|_{\Omega}=f$ e $\left.F\right|_{\partial \Omega}=g$. (Ver [1].)

Para darmos uma resposta à questão acima partimos do seguinte resultado clássico bem conhecido.

Lema 3.1.7. Dados $f \in \mathrm{C}^{\infty}(\Omega)$ e $g \in \mathrm{C}(\partial \Omega)$ suponhamos que:

(I) $\lim _{x \rightarrow \xi} f(x)=g(\xi), \forall \xi \in \partial \Omega$.

(II) Existe $g_{\xi}^{\alpha}:=\lim _{x \rightarrow \xi} \partial^{\alpha} f(x)$, sempre que $\xi \in \partial \Omega$ e $\alpha \in \mathbb{N}^{n}$. 
Então existe $F \in \mathrm{C}^{\infty}(\bar{\Omega})$ tal que $\left.F\right|_{\Omega}=f$ e $\left.F\right|_{\partial \Omega}=g$.

Prova. Ver [1]

A seguir estenderemos o lema acima à estrutura das funções generalizadas.

Proposição 3.1.8. Dados $f \in \mathcal{G}_{f}(\Omega)$ e $g \in \mathcal{G}_{f}^{b}(\partial \Omega)$ suponhamos que existem representantes $\hat{f}$ e $\hat{g}$ de $f$ e $g$ respectivamente, verificando as quatro condições seguintes:

(I) $\lim _{x \rightarrow \xi} \hat{f}(\varphi, x)=\hat{g}(\varphi, \xi)$, sempre que $\xi \in \partial \Omega$ e $\varphi \in \mathcal{A}_{0}(\mathbb{K})$

(II) Existe $g_{\varphi, \xi}^{\alpha}:=\lim _{x \rightarrow \xi} \partial^{\alpha} \hat{f}(\varphi, x)$, sempre que $\xi \in \partial \Omega, \varphi \in \mathcal{A}_{0}(\mathbb{K})$, e $\alpha \in \mathbb{N}^{n}$.

$(I I I)\left(g^{\alpha}:(\varphi, x) \in \mathcal{A}_{0}(\mathbb{K}) \times \partial \Omega \mapsto g_{\varphi, x}^{\alpha} \in \mathbb{K}\right) \in \mathcal{E}_{f}^{M}(\partial \Omega), \forall \alpha \in \mathbb{N}^{n}$

(IV) Para todo $K \subset \subset \bar{\Omega}$ com $K \cap \Omega \neq \emptyset$, para todo $\alpha \in \mathbb{N}$, para cada seqüência $\left\{\varphi^{\nu}\right\}_{\nu \in \mathbb{N}}$, onde $\varphi^{\nu} \in \mathcal{A}_{\nu}(\mathbb{K}), \forall \nu \in \mathbb{N}$, para toda seqüência $\left\{x_{\nu}\right\}_{\nu \in \mathbb{N}}$ em $\Omega \cap K$ e para cada seqüência $\left\{\varepsilon_{\nu}\right\}_{\nu \in \mathbb{N}} \in I$ com $\varepsilon_{\nu} \downarrow 0$, existe $C>0, M \in \mathbb{N}$ e $\sigma \in I$ verificando

$$
\sup _{\nu \in \mathbb{N}}\left|\partial^{\alpha} \hat{f}\left(\varphi_{\varepsilon_{\nu}}^{\nu}, x_{\nu}\right)\right| \leq C \varepsilon^{-M}, \forall \varepsilon \in I_{\sigma}
$$

Então, existe $F \in \mathcal{G}_{f}(\bar{\Omega})$ tal que $\left.F\right|_{\Omega}=f$ e $\left.F\right|_{\partial \Omega}=g$. Mais precisamente, a função $\hat{F}: \mathcal{A}_{0}(\mathbb{K}) \times \bar{\Omega} \rightarrow \mathbb{K}$ definida por

$$
\hat{F}(\varphi ; x):= \begin{cases}\hat{f}(\varphi, x), & \forall(\varphi, x) \in \mathcal{A}_{0}(\mathbb{K}) \times \Omega \\ \hat{g}(\varphi, x), & \forall(\varphi, x) \in \mathcal{A}_{0}(\mathbb{K}) \times \partial \Omega .\end{cases}
$$

é uma representante de $F$ e, para cada $\alpha \in \mathbb{N}^{n}$, temos

$$
\partial^{\alpha} \hat{F}(\varphi, x):= \begin{cases}\partial^{\alpha} \hat{f}(\varphi, x), & \forall(\varphi, x) \in \mathcal{A}_{0}(\mathbb{K}) \times \Omega \\ g_{\varphi, x}^{\alpha}, & \forall(\varphi, x) \in \mathcal{A}_{0}(\mathbb{K}) \times \partial \Omega .\end{cases}
$$

Prova. É uma fácil generalização do Lema 3.1.7 (ver [1]).

Corolário 3.1.9. Sejam $\hat{f}$ e $\hat{G}$ representantes arbitrários de $f \in \mathcal{G}_{f}(\Omega)$ dada e $g \in \mathcal{G}_{f}^{b}(\partial \Omega)$ respectivamente. Suponhamos que as seguintes condições valem:

(a) Existe $g_{\varphi, \xi}^{\alpha}:=\lim _{x \rightarrow \xi} \partial^{\alpha} \hat{f}(\varphi, x)$, sempre que $\xi \in \partial \Omega, \varphi \in \mathcal{A}_{0}(\mathbb{K})$ e $\alpha \in \mathbb{N}^{n}$. 
(b) $\left(g^{\alpha}:(\varphi, x) \in \mathcal{A}_{0}(\mathbb{K}) \times \partial \Omega \mapsto g_{\varphi, x}^{\alpha} \in \mathbb{K}\right) \in \mathcal{E}_{f}^{M}(\partial \Omega)$, para cada $\alpha \in \mathbb{N}^{n}$.

(c) $g^{0}-\hat{G} \in \mathcal{N}_{f}(\partial \Omega)$, onde $g^{0}=g^{\alpha}$ de (b) quando $\alpha=0 \in \mathbb{N}^{\alpha}$.

(d) A condição (IV) da Proposição 3.1.9.

Então existe $F \in \mathcal{G}_{f}(\dot{\Omega})$ tal que $\left.F\right|_{\Omega}=f$ e $\left.F\right|_{\partial \Omega}=g$.

Prova. Ver [1]

Seja $X$ um conjunto quase-regular em $\mathbb{R}^{n}$ e $\Omega$ um subconjunto aberto em $\mathbb{R}^{m}$, podemos definir $\mathcal{G}_{f}(\partial \Omega \times X)($ Ver [10], Teorema 1) do seguinte modo. Primeiro introduzimos o anel das funções moderada sobre $\partial \Omega \times X$ que é

$$
\begin{aligned}
\mathcal{E}_{f}^{M}(\partial \Omega \times X):= & \left\{u: \mathcal{A}_{0}(\mathbb{K}) \times \partial \Omega \times X \rightarrow \mathbb{K} \mid \text { para cada } K \subset \subset X, \alpha \in \mathbb{N}^{n} \text { e } H \subset \subset \partial \Omega\right. \\
& \text { existe } N \in \mathbb{N} \text { tal que, para toda } \varphi \in \mathcal{A}_{N}(\mathbb{K}) \text { podemos encontrar } \\
& c=c(\varphi)>0 \text { e } \eta=\eta(\varphi) \in] 0,1] \text { satisfazendo }\left|\partial_{x}^{\alpha} u\left(\varphi_{\varepsilon}, \xi, x\right)\right| \leq c \varepsilon^{-N} \\
& \text { para todo } \left.x \in K, \xi \in H \text { e } \varepsilon \in I_{\eta}\right\} .
\end{aligned}
$$

A seguir consideramos o ideal de $\mathcal{E}_{f}^{M}(\partial \Omega \times X)$ definido por

$$
\begin{aligned}
\mathcal{N}_{f}(\partial \Omega \times X):=\{ & \left\{u \in \mathcal{E}_{f}^{M}(\partial \Omega \times X) \mid \text { para cada } K \subset \subset X, \alpha \in \mathbb{N}^{n} \text { e } H \subset \subset \partial \Omega\right. \\
& \text { existe } N \in \mathbb{N} \text { e } \gamma \in \Gamma \text { tal que, para todo } q \in \mathbb{N} \text { e toda } \varphi \in \mathcal{A}_{q}(\mathbb{K}) \\
& \text { podemos encontrar } c=c(\varphi)>0 \text { e } \eta=\eta(\varphi) \in] 0,1] \text { satisfazendo } \\
& \left.\left|\partial_{x}^{\alpha} u\left(\varphi_{\varepsilon}, \xi, x\right)\right| \leq c \varepsilon^{\gamma(q)-p} \text { para todo } x \in K, \xi \in H \text { e } \varepsilon \in I_{\eta}\right\} .
\end{aligned}
$$

Definição 3.1.10. Seja $X$ um conjunto quase-regular em $\mathbb{R}^{n}$ e $\Omega$ um conjunto aberto em $\mathbb{R}^{m}$. A álgebra das funções generalizadas sobre $\partial \Omega \times X$ é

$$
\mathcal{G}_{f}(\partial \Omega \times X):=\mathcal{E}_{f}^{M}(\partial \Omega \times X) / \mathcal{N}_{f}(\partial \Omega \times X) .
$$

Exemplo 3.1.11. Álgebras de funções generalizadas sobre conjuntos quase-regulares $e$ conceitos relatados aparecem em [10].

Para mais exemplos e aplicações dos conceitos acima indicamos a leitura do trabalho original do Aragona [1]. 


\section{$3.2 \quad$ A topologia cortante sobre $\mathcal{G}_{f}(\Omega)$}

A definição da topologia que daremos é uma forma alternativa daquela dada no Teorema 3.6 de Aragona, Fernandez e Juriaans [3]. Iniciamos, então com a seguinte:

Definição 3.2.1. Seja $\left\{\Omega_{m}\right\}_{m \in \mathbb{N}}$ uma exaustão aberta de $\Omega$. Dado $u \in \mathcal{E}_{f}^{M}(\Omega)$ e $(m, p) \in$ $\mathbb{N}^{2}$ definimos:

$$
\mathrm{A}_{m p}(u):=\left\{r \in \mathbb{R}\left|\forall \beta \in \mathbb{N}^{n},\right| \beta \mid \leq p \text { tem-se } \dot{\alpha}_{-r} u_{m}^{\beta} \approx 0\right\}
$$

onde $u_{m}^{\beta}$ é como definido no Lema 3.1.3 (i). Definimos a "valuação" de u o supremo de $\mathrm{A}_{m p}(u)$, isto é,

$$
\mathrm{V}_{m p}(u)=\sup \left(\mathrm{A}_{m p}(u)\right)
$$

O seguinte lema caracteriza os elementos de $A_{m p}(u)$ e será fundamental para provarmos algumas propriedades da valuação de $u$, isto é, de $\mathrm{V}_{m p}(u)$.

Lema 3.2.2. $r \in \mathrm{A}_{m p}(u)$ se, e somente se, para todo $\beta \in \mathbb{N}^{n}$, com $|\beta| \leq p$, existe $N \in \mathbb{N}$ tal que

$$
\lim _{\varepsilon \downarrow 0} \varepsilon^{-r} u_{m}^{\beta}\left(\varphi_{\varepsilon}\right)=0, \forall \varphi \in \mathcal{A}_{N}(\mathbb{K})
$$

Prova. $r \in \mathrm{A}_{m p} \Leftrightarrow \forall \beta \in \mathbb{N}^{n}$ com $|\beta| \leq p$ tem-se que $\dot{\alpha}_{-r} u_{m}^{\beta} \approx 0 \Leftrightarrow \exists N \in \mathbb{N}$ tal que

$$
\begin{aligned}
\lim _{\varepsilon \rightarrow 0} \dot{\alpha}_{-r}\left(\varphi_{\varepsilon}\right) u_{m}^{\beta}\left(\varphi_{\varepsilon}\right)=0, \forall \varphi \in \mathcal{A}_{N}(\mathbb{K}) & \Leftrightarrow \lim _{\varepsilon \downarrow 0}(i(\varphi))^{-r} \varepsilon^{-r} u_{m}^{\beta}\left(\varphi_{\varepsilon}\right)=0, \forall \varphi \in \mathcal{A}_{N}(\mathbb{K}) \\
& \Leftrightarrow \lim _{\varepsilon \downarrow 0} \varepsilon^{-r} u_{m}^{\beta}\left(\varphi_{\varepsilon}\right)=0, \forall \varphi \in \mathcal{A}_{N}(\mathbb{K}),
\end{aligned}
$$

pois $(i(\varphi))^{-r}>0$ e não depende de $\varepsilon$. Portanto, segue o resultado.

Proposição 3.2.3. Se $a \in \mathrm{A}_{m p}(u)$ e $b<a$, então $b \in \mathrm{A}_{m p}(u)$.

Prova. Pelo Lema (3.2.2) é suficiente mostrarmos que para todo $\beta \in \mathbb{N}^{n}$, com $|\beta| \leq p$, existe $N \in \mathbb{N}$ tal que

$$
\lim _{\varepsilon \downarrow 0} \varepsilon^{-b} u_{m}^{\beta}\left(\varphi_{\varepsilon}\right)=0, \forall \varphi \in \mathcal{A}_{N}(\mathbb{K}) .
$$

Ora, como $a \in \mathrm{A}_{m p}(u)$ segue pelo Lema 3.2 .2 que para todo $\beta \in \mathbb{N}^{n},|\beta| \leq p$, existe $N \in \mathbb{N}$ tal que

$$
\lim _{\varepsilon \downarrow 0} \varepsilon^{-a} u_{m}^{\beta}\left(\varphi_{\varepsilon}\right)=0, \forall \varphi \in \mathcal{A}_{N}(\mathbb{K})
$$


Agora, para este $N \in \mathbb{N}$, sendo $b<a$ e $0<\varepsilon<1$ segue que $\varepsilon^{b}>\varepsilon^{a} \Rightarrow \varepsilon^{-b}<\varepsilon^{-a}$ e, portanto,

$$
\varepsilon^{-b} u_{m}^{\beta}\left(\varphi_{\varepsilon}\right)<\varepsilon^{-a} u_{m}^{\beta}\left(\varphi_{\varepsilon}\right)
$$

pois $u_{m}^{\beta} \in \overline{\mathbb{R}}_{f_{+}}$. E, assim, temos que

$$
\lim _{\varepsilon \downarrow 0} \varepsilon^{-b} u_{m}^{\beta}\left(\varphi_{\varepsilon}\right)<\lim _{\varepsilon \downarrow 0} \varepsilon^{-a} u_{m}^{\beta}\left(\varphi_{\varepsilon}\right)=0, \forall \varphi \in \mathcal{A}_{N}(\mathbb{K}) .
$$

E, portanto, para todo $\beta \in \mathbb{N}^{n}$ com $|\beta| \leq p$, existe $N \in \mathbb{N}$ tal que

$$
\lim _{\varepsilon \downarrow 0} \varepsilon^{-b} u_{m}^{\beta}\left(\varphi_{\varepsilon}\right)=0, \forall \varphi \in \mathcal{A}_{N}(\mathbb{K})
$$

como queríamos mostrar.

Estamos áptos a enumerar algumas propriedades de $\mathrm{V}_{m p}$. Isto fazemos na seguinte:

Proposição 3.2.4. Para todas $u, v \in \mathcal{E}_{f}^{M}(\Omega),(m, p) \in \mathbb{N}^{2} \quad e \lambda \in \mathbb{K}$, temos:

(i) $\mathrm{V}_{m p}(\lambda u)=\mathrm{V}_{m p}(u)$, se $\lambda \neq 0$;

(ii) $\mathrm{V}_{m p}(u v) \geq \mathrm{V}_{m p}(u)+\mathrm{V}_{m p}(v)$;

(iii) $\mathrm{V}_{m p}\left(\dot{\alpha}_{r} u\right)=r+\mathrm{V}_{m p}(u), \forall r \in \mathbb{R}$;

(iv) $\mathrm{V}_{m p}(u+v) \geq \inf \left\{\mathrm{V}_{m p}(u), \mathrm{V}_{m p}(v)\right\}$

(v) $u \in \mathcal{N}_{f}(\Omega) \Leftrightarrow \mathrm{A}_{m p}(u)=\mathbb{R}, \forall(m, p) \in \mathbb{N}^{2} \Leftrightarrow \mathrm{V}_{m p}(u)=+\infty, \forall(m, p) \in \mathbb{N}^{2}$.

(vi) $\mathrm{V}_{m p}$ é constante sobre cada classe de equivalência módulo $\mathcal{N}_{f}(\Omega)$.

Prova. (i) Pelo Lema 3.2.2 temos que $r \in \mathrm{A}_{m p}(\lambda u)$ se, e somente se, para todo $\beta \in \mathbb{N}^{n}$ $\operatorname{com}|\beta| \leq p, \exists N \in \mathbb{N}$ tal que

$$
\lim _{\varepsilon \downarrow 0}(\lambda u)_{m}^{\beta}\left(\varphi_{\varepsilon}\right) \varepsilon^{-r}=0, \forall \varphi \in \mathcal{A}_{N}(\mathbb{K}),
$$

mas

$$
\begin{aligned}
(\lambda u)_{m}^{\beta}\left(\varphi_{\varepsilon}\right) & =\left\|\partial^{\beta}(\lambda u)\left(\varphi_{\varepsilon} \cdot\right)\right\|_{m} \\
& =\left\|\lambda \partial^{\beta} u\left(\varphi_{\varepsilon} \cdot\right)\right\|_{m} \\
& =|\lambda|\left\|\partial^{\beta} u\left(\varphi_{\varepsilon} \cdot\right)\right\|_{m} \\
& =|\lambda| u_{m}^{\beta}\left(\varphi_{\varepsilon}\right)
\end{aligned}
$$


e, portanto, temos

$$
(\lambda u)_{m}^{\beta}\left(\varphi_{\varepsilon}\right)=|\lambda| u_{m}^{\beta}\left(\varphi_{\varepsilon}\right)
$$

Agora, substituindo (3.7) no primeiro membro de (3.6), temos

$$
\lim _{\varepsilon \downarrow 0}|\lambda| u_{m}^{\beta}\left(\varphi_{\varepsilon}\right) \varepsilon^{-r}=0, \forall \varphi \in \mathcal{A}_{N}(\mathbb{K})
$$

se, e somente se,

$$
\lim _{\varepsilon \downarrow 0} u_{m}^{\beta}\left(\varphi_{\varepsilon}\right) \varepsilon^{-r}=0, \forall \varphi \in \mathcal{A}_{N}(\mathbb{K})
$$

pois $\lambda \neq 0 \Leftrightarrow|\lambda| \neq 0$. E, pelo Lema 3.2.2, temos que (3.8) ocorre se, e somente se, $r \in \mathrm{A}_{m p}(u)$. Logo, $\mathrm{A}_{m p}(\lambda u)=\mathrm{A}_{m p}(u)$ e consequentemente, temos que $\mathrm{V}_{m p}(\lambda u)=\mathrm{V}_{m p}(u)$.

(ii) Seja $a<\mathrm{V}_{m p}(u)$ e $b<\mathrm{V}_{m p}(v)$. Então $a<\sup \left(\mathrm{A}_{m p}(u)\right)$ e $b<\sup \left(\mathrm{A}_{m p}(v)\right)$. Agora, pela Proposição 3.2.3, temos que $a \in \mathrm{A}_{m p}(u)$ e $b \in \mathrm{A}_{m p}(v)$. E, pelo Lema 3.2.2, se, e somente se, para todo $\beta \in \mathbb{N}^{n}$ com $|\beta| \leq p$ existe $N \in \mathbb{N}$ tal que

$$
\lim _{\varepsilon \downarrow 0} \varepsilon^{-a} u_{m}^{\beta}\left(\varphi_{\varepsilon}\right)=0=\lim _{\varepsilon \downarrow 0} \varepsilon^{-b} v_{m}^{\beta}\left(\varphi_{\varepsilon}\right), \forall \varphi \in \mathcal{A}_{N}(\mathbb{K})
$$

Como pelo Lema 3.1.3

$$
u_{m}^{\beta}\left(\varphi_{\varepsilon}\right)=\left\|\partial^{\beta} u\left(\varphi_{\varepsilon}, \cdot\right)\right\|_{m} \quad \text { e } \quad v_{m}^{\beta}\left(\varphi_{\varepsilon}\right)=\left\|\partial^{\beta} v\left(\varphi_{\varepsilon}, \cdot\right)\right\|_{m},
$$

podemos escrever (3.9) como segue:

$$
\lim _{\varepsilon \downarrow 0} \frac{\left\|\partial^{\beta} u\left(\varphi_{\varepsilon}, \cdot\right)\right\|_{m}}{\varepsilon^{a}}=0=\lim _{\varepsilon \downarrow 0} \frac{\left\|\partial^{\beta} v\left(\varphi_{\varepsilon}, \cdot\right)\right\|_{m}}{\varepsilon^{b}}, \forall \varphi \in \mathcal{A}_{N}(\mathbb{K}) .
$$

Agora, pela regra de Leibnitz, sendo

$$
\frac{\partial^{\beta}(u v)}{\varepsilon^{a+b}}=\sum_{\beta^{\prime} \leq \beta}\left(\begin{array}{c}
\beta \\
\beta^{\prime}
\end{array}\right) \frac{\partial^{\beta-\beta^{\prime}} u}{\varepsilon^{a}} \frac{\partial^{\beta^{\prime}} v}{\varepsilon^{b}},\left|\beta-\beta^{\prime}\right| \leq p \text { e }\left|\beta^{\prime}\right| \leq p
$$

segue que

$$
\lim _{\varepsilon \downarrow 0} \frac{\left\|\partial^{\beta}(u v)\left(\varphi_{\varepsilon}, \cdot\right)\right\|_{m}}{\varepsilon^{a+b}}=0, \forall \varphi \in \mathcal{A}_{N}(\mathbb{K}) .
$$

Mas, novamente, pelo Lema 3.1.3, temos que

$$
\left\|\partial^{\beta}(u v)\left(\varphi_{\varepsilon}, \cdot\right)\right\|_{m}=(u v)_{m}^{\beta}\left(\varphi_{\varepsilon}\right)
$$

e, portanto, podemos escrever 3.11, assim:

$$
\lim _{\varepsilon \downarrow 0} \varepsilon^{-(a+b)}(u v)_{m}^{\beta}\left(\varphi_{\varepsilon}\right)=0, \forall \varphi \in \mathcal{A}_{N}(\mathbb{K}) .
$$


$\mathrm{E}$, pelo Lema 3.2.2, (3.12) ocorre se, e somente se, $a+b \in \mathrm{A}_{m p}(u v)$ e, portanto,

$$
\mathrm{V}_{m p}(u v) \geq a+b
$$

Como $a<\mathrm{V}_{m p}(u)$ e $b<\mathrm{V}_{m p}(v)$ são quaisquer, temos que

$$
\mathrm{V}_{m p}(u v) \geq \mathrm{V}_{m p}(u)+\mathrm{V}_{m p}(v)
$$

(iii) Pelo Lema 3.2.2 a $\in \mathrm{A}_{m p}\left(\dot{\alpha}_{r} u\right)$ se, e somente se, para todo $\beta \in \mathbb{N}^{n}$ com $|\beta| \leq p$ existe $N \in \mathbb{N}$ tal que

$$
\lim _{\varepsilon \downarrow 0} \varepsilon^{-a}\left(\dot{\alpha}_{r} u\right)_{m}^{\beta}\left(\varphi_{\varepsilon}\right)=0, \forall \varphi \in \mathcal{A}_{N}(\mathbb{K}) .
$$

Mas, pelo Lema 3.1.3, temos que

$$
\begin{aligned}
\left(\dot{\alpha}_{r} u\right)_{m}^{\beta}\left(\varphi_{\varepsilon}\right) & =\left\|\partial^{\beta}\left(\dot{\alpha}_{r} u\right)\left(\varphi_{\varepsilon}, \cdot\right)\right\|_{m} \\
& =\left\|\dot{\alpha}_{r}\left(\varphi_{\varepsilon}\right) \partial^{\beta} u\left(\varphi_{\varepsilon}, \cdot\right)\right\|_{m} \\
& =\dot{\alpha}_{r}\left(\varphi_{\varepsilon}\right)\left\|\partial^{\beta} u\left(\varphi_{\varepsilon}, \cdot\right)\right\|_{m} \\
& =\varepsilon^{r}(i(\varphi))^{r}\left\|\partial^{\beta} u\left(\varphi_{\varepsilon}, \cdot\right)\right\|_{m} \\
& =\varepsilon^{r}(i(\varphi))^{r} u_{m}^{\beta}\left(\varphi_{\varepsilon}\right)
\end{aligned}
$$

e, portanto, temos que

$$
\left(\dot{\alpha}_{r} u\right)_{m}^{\beta}\left(\varphi_{\varepsilon}\right)=\varepsilon^{r}(i(\varphi))^{r} u_{m}^{\beta}\left(\varphi_{\varepsilon}\right) .
$$

Agora, substituindo-se (3.14) em (3.13), vem

$$
0=\lim _{\varepsilon \downarrow 0} \varepsilon^{-a} \varepsilon^{r}(i(\varphi))^{r} u_{m}^{\beta}\left(\varphi_{\varepsilon}\right)=(i(\varphi))^{r} \lim _{\varepsilon \downarrow 0} \varepsilon^{-(a-r)} u_{m}^{\beta}\left(\varphi_{\varepsilon}\right), \forall \varphi \in \mathcal{A}_{N}(\mathbb{K})
$$

se, e somente se,

$$
\lim _{\varepsilon \downarrow 0} \varepsilon^{-(a-r)} u_{m}^{\beta}\left(\varphi_{\varepsilon}\right)=0, \forall \varphi \in \mathcal{A}_{N}(\mathbb{K}),
$$

pois $(i(\varphi))^{r}>0, \forall \varphi \in \mathcal{A}_{N}(\mathbb{K})$. E, novamente, pelo Lema (3.2.2), temos que (3.15) ocorre se, e somente se, $a-r \in \mathrm{A}_{m p}(u) \Leftrightarrow \exists z \in \mathrm{A}_{m p}(u)$ tal que $z=a-r \Leftrightarrow a=z+r$. E, portanto,

$$
\mathrm{V}_{m p}\left(\dot{\alpha}_{r} u\right)=\mathrm{V}_{m p}(u)+r
$$

(iv) Seja $a<\inf \left\{\mathrm{V}_{m p}(u), \mathrm{V}_{m p}(v)\right\}$. Então

$$
a<\mathrm{V}_{m p}(u)=\sup \left(\mathrm{A}_{m p}(u)\right) \text { e } a<\mathrm{V}_{m p}(v)=\sup \left(\mathrm{A}_{m p}(v)\right)
$$


assim, pela Proposição 3.2.3, temos que $a \in \mathrm{A}_{m p}(u)$ e $a \in \mathrm{A}_{m p}(v)$. Agora, pelo Lema 3.2.2 isto ocorre se, e somente se, para todo $\beta \in \mathbb{N}^{n}$ com $|\beta| \leq p$ existe $N \in \mathbb{N}$ tal que

$$
\lim _{\varepsilon \downarrow 0} \varepsilon^{-a} u_{m}^{\beta}\left(\varphi_{\varepsilon}\right)=0=\lim _{\varepsilon \downarrow 0} \varepsilon^{-a} v_{m}^{\beta}\left(\varphi_{\varepsilon}\right), \forall \varphi \in \mathcal{A}_{N}(\mathbb{K})
$$

Como, pelo Lema 3.1.3, temos

$$
u_{m}^{\beta}\left(\varphi_{\varepsilon}\right)=\left\|\partial^{\beta} u\left(\varphi_{\varepsilon}, \cdot\right)\right\|_{m} \text { e } v_{m}^{\beta}\left(\varphi_{\varepsilon}\right)=\left\|\partial^{\beta} v\left(\varphi_{\varepsilon}, \cdot\right)\right\|_{m}
$$

podemos escrever (3.16) como segue:

$$
\lim _{\varepsilon \downarrow 0} \frac{\left\|\partial^{\beta} u\left(\varphi_{\varepsilon}, \cdot\right)\right\|_{m}}{\varepsilon^{a}}=0=\lim _{\varepsilon \downarrow 0} \frac{\left\|\partial^{\beta} v\left(\varphi_{\varepsilon}, \cdot\right)\right\|_{m}}{\varepsilon^{a}}, \forall \varphi \in \mathcal{A}_{N}(\mathbb{K}) .
$$

Portanto,

$$
\lim _{\varepsilon \downarrow 0} \frac{\left\|\partial^{\beta}(u+v)\left(\varphi_{\varepsilon}, \cdot\right)\right\|_{m}}{\varepsilon^{a}} \leq \lim _{\varepsilon \downarrow 0} \frac{\left\|\partial^{\beta} u\left(\varphi_{\varepsilon}, \cdot\right)\right\|_{m}}{\varepsilon^{a}}+\lim _{\varepsilon \downarrow 0} \frac{\left\|\partial^{\beta} v\left(\varphi_{\varepsilon}, \cdot\right)\right\|_{m}}{\varepsilon^{a}}=0, \forall \varphi \in \mathcal{A}_{N}(\mathbb{K}),
$$

isto é,

$$
\lim _{\varepsilon \downarrow 0} \frac{\left\|\partial^{\beta}(u+v)\left(\varphi_{\varepsilon}, \cdot\right)\right\|_{m}}{\varepsilon^{a}}=0, \forall \varphi \in \mathcal{A}_{N}(\mathbb{K}) .
$$

E, novamente, pelo Lema 3.1.3 podemos escrever (3.18) assim:

$$
\lim _{\varepsilon \downarrow 0} \varepsilon^{-a}(u+v)_{m}^{\beta}=0, \forall \varphi \in \mathcal{A}_{N}(\mathbb{K}) .
$$

Agora, pelo Lema 3.2.2, temos que (3.19) ocorre se, e somente se, $a \in \mathrm{A}_{m p}(u+v)$ e, portanto,

$$
a \leq \sup \left(\mathrm{A}_{m p}(u+v)\right)=\mathrm{V}_{m p}(u+v) .
$$

(v) Temos que $u \in \mathcal{N}_{f}(\Omega)$ se, e somente se, $\forall(m, p) \in \mathbb{N}^{2} \forall r \in \mathbb{R}, \exists N \in \mathbb{N}$ tal que $\forall \varphi \in \mathcal{A}_{N}(\mathbb{K})$ temos $\left\|\partial^{\beta} \dot{u}\left(\varphi_{\varepsilon}, \cdot\right)\right\|_{m}=o\left(\varepsilon^{r}\right)$, se $\varepsilon \downarrow 0, \forall|\beta| \leq p$ (ver Proposição 3.1.1) se, e somente se, $\forall(m, p) \in \mathbb{N}^{2} \forall r \in \mathbb{R}, \exists N \in \mathbb{N}$ tal que $\forall \varphi \in \mathcal{A}_{N}(\mathbb{K})$ temos $u_{m}^{\beta}\left(\varphi_{\varepsilon}\right)=o\left(\varepsilon^{r}\right)$ se $\varepsilon \downarrow 0, \forall|\beta| \leq p$, pois $\left\|\partial^{\beta} u\left(\varphi_{\varepsilon}, \cdot\right)\right\|_{m}=u_{m}^{\beta}\left(\varphi_{\varepsilon}\right)$ (ver Lema 3.1.3) se, e somente se, $\forall(m, p) \in \mathbb{N}^{2} \forall r \in \mathbb{R}, \exists N \in \mathbb{N}$ tal que $\forall \varphi \in \mathcal{A}_{N}(\mathbb{K})$ temos que

$$
\lim _{\varepsilon \downarrow 0} u_{m}^{\beta}\left(\varphi_{\varepsilon}\right) \varepsilon^{-r}=0, \forall|\beta| \leq p
$$

se, e somente se, $r \in \mathrm{A}_{m p}(u)$, (ver Lema 3.2.2) se, e somente se, $\mathrm{A}_{m p}(u)=\mathbb{R}, \forall(m, p) \in \mathbb{N}^{2}$ (pois $\mathbb{R} \subset \mathrm{A}_{m p}(u)$ e $\mathrm{A}_{m p}(u) \subset \mathbb{R}$ ) se, e somente se,

$$
+\infty=\sup \left(\mathrm{A}_{m p}(u)\right)=\mathrm{V}_{m p}(u), \forall(m, p) \in \mathbb{N}^{2}
$$

(ver Definição 3.2 .1 da valuação de $u$ ). E, portanto, mostramos $(v$ ).

(vi) É fácil e deixamos para o leitor! 
Proposição 3.2.5. A função $D_{m p}: \mathcal{G}_{f}(\Omega) \times \mathcal{G}_{f}(\Omega) \rightarrow \mathbb{R}_{+}$definida por

$$
D_{m p}(f, g)=\exp \left(-\mathrm{V}_{m p}(\hat{f}-\hat{g})\right)
$$

onde $\hat{f}$ e $\hat{g}$ são representantes quaisquer de $f$ e g, respectivamente, é uma pseudo-ultramétrica sobre $\mathcal{G}_{f}(\Omega)$.

Prova. Para mostrarmos isto, precisamos mostrar que para toda $f, g, h \in \mathcal{G}_{f}(\Omega)$, temos:

(i) $D_{m p}(f, g) \geq 0$

(ii) $D_{m p}(f, g)=D_{m p}(g, f)$

(iii) $D_{m p}(f, f)=0$

(iv) $D_{m p}(f, g) \leq \max \left\{D_{m p}(f, h), D_{m p}(h, g)\right\}$

(i) Temos que $D_{m p}(f, g)=e^{-\mathrm{V}_{m p}(\hat{f}-\hat{g})} \geq 0$ e $D_{m p}(f, g)=0 \Leftrightarrow e^{-\mathrm{V}_{m p}(\hat{f}-\hat{g})}=0 \Leftrightarrow$ $\mathrm{V}_{m p}(\hat{f}-\hat{g})=+\infty \Leftrightarrow \hat{f}-\hat{g} \in \mathcal{N}_{f}(\Omega) \Leftrightarrow \hat{f}=\hat{g} \Leftrightarrow f=g$. E, portanto, mostramos (iii), i.e., $D_{m p}(f, f)=0$. Observe que para mostrarmos (iii) usamos fortemente a Proposição $3.2 .4(v)$.

(ii) Temos que

$$
\begin{aligned}
D_{m p}(f, g) & =e^{-\mathrm{V}_{m p}(\hat{f}-\hat{g})} \\
& =e^{-\mathrm{V}_{m p}((-1)(\hat{g}-\hat{f}))} \text { (pela Proposição 3.2.4 }(i), \text { temos) } \\
& =e^{-\mathrm{V}_{m p}(\hat{g}-\hat{f})} \\
& =D_{m p}(g, f) .
\end{aligned}
$$

E, portanto, $D_{m p}(f, g)=D_{m p}(g, f), \forall f, g \in \mathcal{G}_{f}(\Omega)$.

(iv) Observe que podemos escrever $\hat{f}-\hat{g}$ como segue:

$$
\hat{f}-\hat{g}=(\hat{f}-\hat{h})+(\hat{h}-\hat{g}) \text {. }
$$

Agora, aplicando-se $\mathrm{V}_{m p}$ a ambos os lados da expressão acima e usando a Proposição 3.2.4 (iv), obtemos

$$
\mathrm{V}_{m p}(\hat{f}-\hat{g}) \geq \inf \left\{\mathrm{V}_{m p}(\hat{f}-\hat{h}), \mathrm{V}_{m p}(\hat{h}-\hat{g}\}\right.
$$


Portanto, multiplicando-se ambos os membros desta última expressão por $(-1)$, obtemos

$$
-\mathrm{V}_{m p}(\hat{f}-\hat{g}) \leq \sup \left\{-\mathrm{V}_{m p}(\hat{f}-\hat{h}),-\mathrm{V}_{m p}(\hat{h}-\hat{g}\}\right.
$$

Assim, temos que

$$
\begin{aligned}
e^{-\mathrm{V}_{m p}(\hat{f}-\hat{g})} & \leq e^{\sup \left\{-\mathrm{V}_{m p}(\hat{f}-\hat{h}),-\mathrm{V}_{m p}(\hat{h}-\hat{g}\}\right.} \\
& \leq \sup \left\{e^{-\mathrm{V}_{m p}(\hat{f}-\hat{h})}, e^{-\mathrm{V}_{m p}(\hat{h}-\hat{g})}\right\}
\end{aligned}
$$

e, portanto, pela definição de $D_{m p}$, temos que

$$
D_{m p}(f, g) \leq \sup \left\{D_{m p}(f, h), D_{m p}(h, g)\right\}=\max \left\{D_{m p}(f, h), D_{m p}(h, g)\right\}
$$

e segue a assertiva.

A estrutura uniforme determinada pela família $\left\{D_{m p}\right\}_{(m, p) \in \mathbb{N}^{2}}$ é chamada a estrutura uniforme cortante sobre $\mathcal{G}_{f}(\Omega)$ e, a denotaremos por $\tau_{\Omega f}$. A proposição seguinte garante que $\tau_{\Omega f}$ é Hausdorff, mais precisamente, temos:

Proposição 3.2.6. Para todo $(m, p) \in \mathbb{N}^{2}$ a pseudo-ultra-métrica $D_{m p}$ é invariante por translação. Mais ainda, para toda $f \in \mathcal{G}_{f}(\Omega)$ e $\alpha \in \mathbb{N}^{n}$ as seguintes afirmações se verificam:

(i) $f=0$ se, e somente se, para algum (ou equivalentemente, para todo) representante $\hat{f}$ de $f$ temos que $\mathrm{V}_{m p}(\hat{f})=+\infty$ (i.e., $D_{m p}(f, 0)=0$ ) para todo $(m, p) \in \mathbb{N}^{2}$.

(ii) $D_{m p}\left(\partial^{\alpha} f, 0\right) \leq D_{m(p+|\alpha|)}(f, 0)$, para todo $(m, p) \in \mathbb{N}^{2}$.

Prova. Vamos primeiro mostrar que a pseudo-ultra-métrica $D_{m p}$ é invariante por translação. Isto é, $D_{m p}(f+g, h+g)=D_{m p}(f, h), \forall f, g, h \in \mathcal{G}_{f}(\Omega)$. Ora,

$$
\begin{aligned}
D_{m p}(f+g, h+g) & =e^{-\mathrm{V}_{m p}((\hat{f}+\hat{g})-(\hat{h}+\hat{g}))} \\
& =e^{-\mathrm{V}_{m p}(\hat{f}-\hat{h})} \\
& =D_{m p}(f, h), \forall f, g, h \in \mathcal{G}_{f}(\Omega) .
\end{aligned}
$$

Agora, na seqüência, vamos mostrar (i) e (ii).

(i) Temos que $f=0 \Leftrightarrow f \in \mathcal{N}_{f}(\Omega)$ e pela Proposição 3.2.4 item $(v)$ se, e somente se, $\mathrm{V}_{m p}(\hat{f})=+\infty, \forall(m, p) \in \mathbb{N}^{2}$, onde $\hat{f}$ é um representante qualquer de $f$. Agora, isto é equivalente a dizer que $D_{m p}(f, 0)=e^{-\mathrm{V}_{m p}(\hat{f})}=0$, pois $\mathrm{V}_{m p}(\hat{f})=+\infty, \forall(m, p) \in \mathbb{N}^{2}$. 
(ii) Com efeito, se $a<\mathrm{V}_{m(p+|\alpha|)}(\hat{f})$, então sendo

$$
\mathrm{V}_{m(p+|\alpha|)}(\hat{f})=\sup \left(\mathrm{A}_{m(p+|\alpha|)}(\hat{f})\right)
$$

segue pela Proposição 3.2.3 que $a \in \mathrm{A}_{m(p+|\alpha|)}(\hat{f})$. Então pelo Lema 3.2.2 segue que para todo $\beta \in \mathbb{N}^{n}$ com $|\beta|<p+|\alpha|$ existe $N \in \mathbb{N}$ tal que

$$
\lim _{\varepsilon \downarrow 0} \varepsilon^{-a} \hat{f}_{m}^{\beta}\left(\varphi_{\varepsilon}\right)=0, \forall \varphi \in \mathcal{A}_{N}(\mathbb{K})
$$

Isto é, para todo $\beta \in \mathbb{N}^{n}$ com $|\beta|<p+|\alpha|$, existe $N \in \mathbb{N}$ tal que

$$
\lim _{\varepsilon \downarrow 0} \varepsilon^{-a}\left\|\partial^{\beta} \hat{f}\left(\varphi_{\varepsilon}, \cdot\right)\right\|_{m}=0, \forall \varphi \in \mathcal{A}_{N}(\mathbb{K})
$$

Portanto,

$$
\lim _{\varepsilon \downarrow 0} \varepsilon^{-a}\left\|\partial^{\beta^{\prime}}\left(\partial^{\alpha} \hat{f}\left(\varphi_{\varepsilon}, \cdot\right)\right)\right\|_{m}=0, \forall\left|\beta^{\prime}\right| \leq p,
$$

pois $\left|\beta^{\prime}+\alpha\right| \leq p+|\alpha|$. Assim, para todo $\beta^{\prime} \in \mathbb{N}^{n}$ com $\left|\beta^{\prime}\right|<p, \exists N \in \mathbb{N}$ tal que

$$
\lim _{\varepsilon \downarrow 0} \varepsilon^{-a}\left(\partial^{\alpha} \hat{f}\right)_{m}^{\beta^{\prime}}\left(\varphi_{\varepsilon}\right)=0, \forall \varphi \in \mathcal{A}_{N}(\mathbb{K})
$$

$\mathrm{E}$, novamente, pelo Lema 3.2 .2 , temos que $a \in \mathrm{A}_{m p}\left(\partial^{\alpha} \hat{f}\right)$, assim, mostramos que

$$
\mathrm{A}_{m(p+|\alpha|)}(\hat{f}) \subseteq \mathrm{A}_{m p}\left(\partial^{\alpha} \hat{f}\right)
$$

Agora, aplicando-se o sup a ambos os lados da expressão acima e usando a definição de valuação, temos que

$$
\mathrm{V}_{m(p+|\alpha|)}(\hat{f}) \leq \mathrm{V}_{m p}\left(\partial^{\alpha} \hat{f}\right)
$$

o que implica

$$
e^{-\mathrm{V}_{m(p+|\alpha|)}(\hat{f})} \geq e^{-\mathrm{V}_{m p}\left(\partial^{\alpha} \hat{f}\right)}
$$

e pela definição de $D_{m p}$, temos

$$
D_{m(p+|\alpha|)}(f, 0) \geq D_{m p}\left(\partial^{\alpha} f, 0\right), \forall(m, p) \in \mathbb{N}^{2} .
$$

E isto mostra o que queríamos. 


\subsection{Resultados sobre convergência em $\left(\mathcal{G}_{f}(\Omega), \tau_{\Omega f}\right)$}

Dados $\left.s=\left\{\left(m_{k}, p_{k}\right)\right\}_{1 \leq k \leq \nu}\right\}$, uma seqüência finita em $\mathbb{N}^{2}$ e $\left.a \in\right] 0,1[$ definimos

$$
W_{s}^{a}:=\left\{f \mid \sup _{1 \leq k \leq \nu}\left\{D_{m_{k} p_{k}}(f, 0)\right\}<a\right\}
$$

Então o conjunto $\mathcal{B}$ de todos os $W_{s}^{a}$ (fazendo variar $s \in \mathbb{N}^{2}$ e $\left.a \in\right] 0,1[$ ) é um sistema fundamental de vizinhanças de zero em $\mathcal{G}_{f}(\Omega)$. Além disso, se $B_{r}:=\left\{f \mid D_{m p}(f, 0)<r\right\}$, então é óbvio que

$$
W_{s}^{a}=\bigcap_{1 \leq k \leq \nu} B_{a}^{k}=\bigcap_{1 \leq k \leq \nu}\left\{f \mid D_{m_{k}, p_{k}}(f, 0)<a\right\}
$$

onde $B_{a}^{k}:=\left\{f \mid D_{m_{k} p_{k}}(f, 0)<a\right\}$

Usando o sistema de vizinhanças de zero $\mathcal{B}$ temos a seguinte:

Proposição 3.3.1. Seja a seqüência $\left\{f_{l}\right\}_{l \in \mathbb{N}} \subset \mathcal{G}_{f}(\Omega)$. Então as seguintes afirmações são equivalentes:

(i) $f_{l} \underset{l \rightarrow \infty}{\longrightarrow} f$ em $\mathcal{G}_{f}(\Omega)$;

(ii) para toda seqüência finita $s=\left\{\left(m_{k}, p_{k}\right)\right\}_{1 \leq k \leq \nu} \subset \mathbb{N}^{2}$ e para todo a $\left.\in\right] 0,1[$ existe $l_{0} \in \mathbb{N}$ tal que para todo $l \geq l_{0}$, existem $\left.\left.\varepsilon_{l} \in I=\right] 0,1\right], N_{k} \in \mathbb{N}$ tal que se $0<\varepsilon<\varepsilon_{l}$ $e 1 \leq k \leq \nu$, então $\left\|\partial^{\beta}\left(f_{l}-f\right)\left(\varphi_{\varepsilon}, \cdot\right)\right\|_{m_{k}} \leq \varepsilon^{-\ln a}, \forall \varphi \in \mathcal{A}_{N_{k}}(\mathbb{K}), \forall|\beta| \leq p_{k}$.

Prova. Suponhamos que $(i)$ ocorre, i.e., $f_{l} \underset{l \rightarrow \infty}{\longrightarrow} f$ em $\mathcal{G}_{f}(\Omega)$. Então sendo $\mathcal{B}$ um sistema fundamental de vizinhanças de zero em $\mathcal{G}_{f}(\Omega)$ segue para toda seqüência finita $s=\left\{\left(m_{k}, p_{k}\right)\right\}_{1 \leq k \leq \nu} \subset \mathbb{N}^{2}$ e $\left.a \in\right] 0,1\left[\right.$ existe $l_{0} \in \mathbb{N}$ tal que para todo $l \geq l_{0}$ tem-se que $\left(f_{l}-f\right) \in W_{s}^{a}$. Agora pela definição de $W_{s}^{a}$, temos que

$$
\sup _{1 \leq k \leq \nu}\left\{D_{m_{k} p_{k}}\left(f_{l}-f, 0\right)\right\}<a
$$

isto é,

$$
\sup _{1 \leq k \leq \nu}\left\{\exp \left(-\mathrm{V}_{m_{k} p_{k}}\left(f_{l}-f\right)\right\}<a .\right.
$$

Daí, temos que $\exp \left(-\mathrm{V}_{m_{k} p_{k}}\left(f_{l}-f\right)\right)<a, \forall 1 \leq k \leq \nu$ o que implica

$$
\mathrm{V}_{m_{k} p_{k}}\left(f_{l}-f\right)>-\ln a, \forall 1 \leq k \leq \nu
$$


Pela definição de $\mathrm{V}_{m_{k} p_{k}}$ e a Proposição 3.2.3 segue que - $\ln a \in \mathrm{A}_{m_{k} p_{k}}\left(f_{l}-f\right), \forall 1 \leq k \leq \nu$. Agora, pelo Lema 3.2.2 para todo $\beta \in \mathbb{N}^{n},|\beta| \leq p_{k}(1 \leq k \leq \nu)$ existe $N_{k} \in \mathbb{N}$ tal que

$$
\lim _{\varepsilon \downarrow 0} \varepsilon^{\ln a}\left(f_{l}-f\right)_{m_{k}}^{\beta}\left(\varphi_{\varepsilon}\right)=0, \forall \varphi \in \mathcal{A}_{N}(\mathbb{K})
$$

Como pelo Lema 3.1.3 (i), temos

$$
\left(f_{l}-f\right)_{m_{k}}^{\beta}\left(\varphi_{\varepsilon}\right)=\left\|\partial^{\beta}\left(f_{l}-f\right)\left(\varphi_{\varepsilon}, \cdot\right)\right\|_{m_{k}}
$$

segue que substituindo-se (3.21) em (3.20), obtemos

$$
\lim _{\varepsilon \downarrow 0} \varepsilon^{\ln a}\left\|\partial^{\beta}\left(f_{l}-f\right)\left(\varphi_{\varepsilon}, \cdot\right)\right\|_{m_{k}}=0, \forall \varphi \in \mathcal{A}_{N_{k}}(\mathbb{K}), \forall|\beta| \leq p_{k}
$$

$\mathrm{E}$, portanto, resumindo temos que

$$
f_{l} \underset{l \rightarrow \infty}{\longrightarrow} f \Leftrightarrow\left\{\begin{array}{l}
\left.\forall \text { seqüência finita } s=\left\{\left(m_{k}, p_{k}\right)\right\} \subset \mathbb{N}^{2} \text { e } \forall a \in\right] 0,1\left[\exists l_{0} \in \mathbb{N}\right. \\
\text { tal que } l \geq l_{0} \text { e } 1 \leq k \leq \nu \Rightarrow \forall \beta \in \mathbb{N}^{n}, \text { com }|\beta| \leq p_{k} \\
\exists N_{k} \in \mathbb{N} \text { tal que } \lim _{\varepsilon \downarrow 0} \varepsilon^{\ln a}\left\|\partial^{\beta}\left(f_{l}-f\right)\left(\varphi_{\varepsilon}, \cdot\right)\right\|_{m_{k}}=0, \\
\forall \varphi \in \mathcal{A}_{N_{k}}(\mathbb{K}) .
\end{array}\right.
$$

Finalmente, mostremos que o que esta entre chaves em (3.23) é equivalente a (ii). Fixemos $s=\left\{\left(m_{k}, p_{k}\right)\right\}_{1 \leq k \leq \nu}$ e $a$ como em (ii). Pelo que esta entre chaves em (3.23), associados aos dados acima, podemos encontrar $l_{0} \in \mathbb{N}$ tal que $l \geq l_{0}$ e $1 \leq k \leq \nu$ implica que existe $N_{k} \in \mathbb{N}$ tal que (3.22) se verifica. Assim, $\forall l \geq l_{0}$ e $\forall k=1,2 \ldots, \nu \exists l_{k} \in I$ tal que

$$
\varepsilon^{\ln a}\left\|\partial^{\beta}\left(f_{l}-f\right)\left(\varphi_{\varepsilon}, \cdot\right)\right\|_{m_{k}} \leq 1, \forall \varepsilon \in I_{\varepsilon_{l_{k}}}
$$

Portanto, definindo

$$
\varepsilon_{l}:=\min _{1 \leq k \leq \nu}\left\{\varepsilon_{l_{k}}\right\}
$$

de (3.24) resulta a asserção seguinte (associados aos dados $s=\left\{\left(m_{k}, p_{k}\right)\right\}_{1 \leq k \leq \nu}$ e $a$ existe $\left.l_{0} \in \mathbb{N}\right)$ tal que para todo $l \geq l_{0}$ existem $\varepsilon_{l} \in I$ e $N_{k} \in \mathbb{N}$ tal que $\varepsilon \in I_{\varepsilon_{l}}$ e $1 \leq k \leq \nu$ implica

$$
\left\|\partial^{\beta}\left(f_{l}-f\right)\left(\varphi_{\varepsilon}, \cdot\right)\right\|_{m_{k}} \leq \varepsilon^{-\ln a}, \forall \varphi \in \mathcal{A}_{N_{k}}(\mathbb{K}), \forall|\beta| \leq p_{k}
$$

que é exatamente a afirmação (ii). Vamos agora, para finalizar, mostrar que a afirmação (ii) implica na afirmação que esta entre chaves em (3.23). Fixemos $s=\left\{\left(m_{k}, p_{k}\right)\right\}_{1 \leq k \leq \nu}$ e $a$ como na afirmação que esta entre chaves em (3.23). Como $a^{2} \in$ ] 0,1 [ podemos aplicar 
(ii) aos dados $s=\left\{\left(m_{k}, p_{k}\right)\right\}_{1 \leq k \leq \nu}$ e $a^{2}$ e, portanto, existe $l_{0} \in \mathbb{N}$ tal que para todo $l \geq l_{0}$ existem $\varepsilon_{l} \in I$ e $N_{k} \in \mathbb{N}$ tal que $\varepsilon \in I_{\varepsilon_{l}}$ e $1 \leq k \leq \nu$ implica

$$
\left\|\partial^{\beta}\left(f_{l}-f\right)\left(\varphi_{\varepsilon}, \cdot\right)\right\|_{m_{k}} \leq \varepsilon^{-\ln a^{2}}, \forall \varphi \in \mathcal{A}_{N_{k}}(\mathbb{K}), \forall|\beta| \leq p_{k}
$$

Sendo $\varepsilon^{-\ln a^{2}}=\left(\varepsilon^{-\ln a}\right)^{2}$ a implicação acima pode ser escrita assim: $\varepsilon \in I_{\varepsilon_{l}}$ e $1 \leq k \leq \nu$ implica

$$
\varepsilon^{\ln a}\left\|\partial^{\beta}\left(f_{l}-f\right)\left(\varphi_{\varepsilon}, \cdot\right)\right\|_{m_{k}} \leq \varepsilon^{-\ln a}, \forall . \varphi \in \mathcal{A}_{N_{k}}(\mathbb{K}), \forall|\beta| \leq p_{k} .
$$

Como $a \in] 0,1\left[\therefore-\ln a>0\right.$ segue que $\varepsilon^{-\ln a} \underset{\varepsilon \rightarrow 0}{\longrightarrow} 0$. E, portanto, por (3.26) segue que

$$
\lim _{\varepsilon \downarrow 0} \varepsilon^{\ln a}\left\|\partial^{\beta}\left(f_{l}-f\right)\left(\varphi_{\varepsilon}, \cdot\right)\right\|_{m_{k}}=0, \forall \varphi \in \mathcal{A}_{N_{k}}(\mathbb{K}), \forall|\beta| \leq p_{k}
$$

Assim, provamos que associados aos dados $s=\left\{\left(m_{k}, p_{k}\right)\right\}_{1 \leq k \leq \nu}$ e $a$ existe $l_{0} \in \mathbb{N}$ tal que $l \geq l_{0}$ e $1 \leq k \leq \nu$ implica que existe $N_{k} \in \mathbb{N}$ tal que (3.27) se verifica e, portanto, temos a afirmação entre chaves em (3.23) satisfeita. Assim, mostramos o que queríamos.

Como conseqüência da Proposição 3.3.1, temos que se $\left\{f_{l}\right\}_{l \in \mathbb{N}}$ é uma seqüência em $\mathcal{G}_{f}(\Omega)$ que é $\tau_{\Omega f^{-}}$-convergente, então para todo $\alpha \in \mathbb{N}^{n}$, a seqüência $\left\{\partial^{\alpha} f_{l}\right\}_{l \in \mathbb{N}}$ é $\tau_{\Omega f^{-}}$ convergente. Mais precisamente, temos:

Corolário 3.3.2. Se $f_{l} \underset{l \rightarrow \infty}{\longrightarrow} f$, então para todo $\alpha \in \mathbb{N}^{n}$ temos $\partial^{\alpha} f_{l} \underset{l \rightarrow \infty}{\longrightarrow} \partial^{\alpha} f$. Em outros termos, temos que para todo $\alpha \in \mathbb{N}^{n}$, o operador derivação parcial

$$
\partial^{\alpha}: f \in \mathcal{G}_{f}(\Omega) \mapsto \partial^{\alpha} f \in \mathcal{G}_{f}(\Omega)
$$

é contínuo.

Prova. Pela Proposição 3.3.1 basta fixarmos $s=\left\{\left(m_{k}, p_{k}\right)\right\}_{1 \leq k \leq \nu}$ e $\left.a \in\right] 0,1[$ quaisquer e provar que existe $l_{0} \in \mathbb{N}$ tal que para todo $l \geq l_{0}$ existem $\varepsilon_{l} \in I, N_{k} \in \mathbb{N}$ tal que $0<\varepsilon<\varepsilon_{l}$ e $1 \leq k \leq \nu \Rightarrow\left\|\partial^{\beta+\alpha}\left(f_{l}-f\right)\left(\varphi_{\varepsilon}, \cdot\right)\right\|_{m_{k}} \leq \varepsilon^{-\ln a}, \forall \varphi \in \mathcal{A}_{n}(\mathbb{K}), \forall|\beta| \leq p_{k}$. Mas isto é evidente, pois como $f_{l} \underset{l \rightarrow \infty}{\longrightarrow} f$ por hipótese, basta considerarmos a seqüência $s^{\prime}:=\left\{\left(m_{k}, p_{k}^{\prime}\right)\right\}_{1 \leq k \leq \nu}$, com $p_{k}^{\prime}:=p_{k}+|\alpha|$ já que associados aos dados $s^{\prime}$ e $a$, podemos achar $l_{0} \in \mathbb{N}$ tal que para todo $l \geq l_{0}$ existem $\varepsilon_{l} \in I, N \in \mathbb{N}$ tal que $0<\varepsilon<\varepsilon_{l}$ e $1 \leq k \leq \nu \Rightarrow\left\|\partial^{\gamma}\left(f_{l}-f\right)\left(\varphi_{\varepsilon}, \cdot\right)\right\|_{m_{k}} \leq \varepsilon^{-\ln a}, \forall|\gamma| \leq p_{k}^{\prime}$, pois como $|\beta+\alpha|=|\beta|+|\alpha|$, a definição de $p_{k}^{\prime}$ mostra que $\left\{\beta+\alpha \| \beta \mid \leq p_{k}\right\} \subset\left\{\gamma \| \gamma \mid \leq p_{k}^{\prime}\right\}, \forall k=1,2, \ldots \nu$.

Denotamos por $\mathcal{H G}_{f}(\Omega)$ a álgebra das funções holomorfas generalizadas plenas de Colombeau. Com esta notação temos o seguinte: 
Corolário 3.3.3. Se $\Omega$ é um aberto de $\mathbb{C}^{n}$, então $\mathcal{H G}_{f}(\Omega)$ é uma subálgebra $\tau_{\Omega f}$-fechada $e$, portanto, completa de $\mathcal{G}_{f}(\Omega)$.

Prova. Seja $\left\{f_{n}\right\}_{n \in \mathbb{N}} \subset \mathcal{H G}_{f}(\Omega)$ tal que $f_{n} \underset{n \rightarrow \infty}{\longrightarrow} f \in \mathcal{G}_{f}(\Omega)$ então, pelo Corolário 3.3.2, para todo $j=1,2 \ldots n$, temos

$$
0=\frac{\partial f_{n}}{\partial \bar{z}_{j}} \underset{n \rightarrow \infty}{\longrightarrow} \frac{\partial f}{\partial \bar{z}_{j}}
$$

o que mostra que $\frac{\partial f}{\partial \bar{z}_{j}}=0$.

O Corolário 3.3.3 acima é um caso especial do fato geral a seguir; resultante do Corolário 3.3.2:

Corolário 3.3.4. Se P éum operador diferencial linear com coeficientes constantes, então $o \operatorname{ker}(P)$ é um subespaço fechado de $\mathcal{G}_{f}(\Omega)$.

Proposição 3.3.5. Sejam $\Omega$ eV abertos de $\mathbb{R}^{n}$ tais que $\emptyset \neq V \subset \Omega$. Então, a restrição (que é um homomorfismo de álgebras)

$$
R=R_{V}^{\Omega}:\left.f \in\left(\mathcal{G}_{f}(\Omega), \tau_{\Omega, f}\right) \mapsto f\right|_{V} \in\left(\mathcal{G}_{f}(V), \tau_{V, f}\right)
$$

é contínua.

Prova. Para mostrarmos isto, precisamos mostrar que se $\left\{f_{l}\right\}_{l \in \mathbb{N}} \subset \mathcal{G}_{f}(\Omega)$ é uma seqüência $\tau_{\Omega f}$-convergente, isto é, $f_{l} \underset{l \rightarrow \infty}{\longrightarrow} f$, então $R\left(f_{l}\right) \underset{l \rightarrow \infty}{\longrightarrow} R(f)$. Melhor ainda, $\left.\left.f_{l}\right|_{V} \underset{l \rightarrow \infty}{\longrightarrow} f\right|_{V}$, ou seja $\left\{\left.f_{l}\right|_{V}\right\}_{l \in \mathbb{N}}$ é $\tau_{V f}$-convergente. Pela Proposição 3.3 .1 precisamos mostrar para toda sequeência finita $s=\left\{m_{k}, p_{k}\right\} \subset \mathbb{N}^{2}$ e para todo $\left.\left.a \in\right] 0,1\right]$ existe $l_{0} \in \mathbb{N}$ tal que $l \geq l_{0}$ existe $\left.\left.\varepsilon_{l} \in\right] 0,1\right]$ e $N_{k} \in \mathbb{N}$ tal que se $0<\varepsilon<\varepsilon_{l}$ e $1 \leq k \leq \nu$, então

$$
\left\|\partial^{\beta}\left(\left.f_{l}\right|_{V}-\left.f\right|_{V}\right)\left(\varphi_{\varepsilon}, \cdot\right)\right\|_{V_{m k}} \leq \varepsilon^{-\ln a}, \forall \varphi \in \mathcal{A}_{N_{k}}, \forall|\beta| \leq p_{k} .
$$

Fixemos $s$ e a como acima. Sabendo-se que $V_{m} \subset \bar{V}_{m} \subset \subset \subset \Omega, \forall m \in \mathbb{N}$ segue que existe $q \in \mathbb{N}$ tal que $V_{m_{k}} \subset \Omega_{q}, \forall k=1,2, \ldots, \nu$. Definimos, então $s^{\prime}:=(q, p)$ com $p:=\max _{1 \leq k \leq \nu}\left\{p_{k}\right\}$ e, portanto, associados aos dados $s^{\prime}$ e $a$ temos, por hipótese, que existe $l_{0} \in \mathbb{N}$ tal que para todo $l \geq l_{0}$ existe $\left.\left.\varepsilon_{l} \in\right] 0,1\right]$ e $N_{k} \in \mathbb{N}$ tal que se $0<\varepsilon<\varepsilon_{l}$ e $1 \leq k \leq \nu$, então

$$
\left\|\partial^{\beta}\left(f_{l}-f\right)\left(\varphi_{\varepsilon}, \cdot\right)\right\|_{\Omega_{q}} \leq \varepsilon^{-\ln a}, \forall \varphi \in \mathcal{A}_{N_{k}}(\mathbb{K}), \forall|\beta| \leq p .
$$


Assim, temos que se $0<\varepsilon<\varepsilon_{l}$ e $1 \leq k \leq \nu$, então

$$
\left\|\partial^{\beta}\left(\left.f_{l}\right|_{V}-\left.f\right|_{V}\right)\left(\varphi_{\varepsilon}, \cdot\right)\right\|_{V_{m k}} \leq\left\|\partial^{\beta}\left(f_{l}-f\right)\left(\varphi_{\varepsilon}, \cdot\right)\right\|_{\Omega_{q}}
$$

para cada $\beta \in \mathbb{N}^{n}$, o que junto com (3.29), mostrar (3.28), pois $p \geq p_{k}, \forall 1 \leq k \leq \nu$.

Lembrando o seguinte fato trivial de Topologia Geral:

Proposição 3.3.6. Se $X$ e $Y$ são espaços topológicos, $f \in \mathrm{C}(X ; Y), X_{0}$ e $Y_{0}$ são subespaços topológicos de $X$ e $Y$, respectivamente, tais que $f\left(X_{0}\right) \subset Y_{0}$, então $g:=\left.f\right|_{X_{0}} \in$ $\mathrm{C}\left(X_{0} ; Y_{0}\right)$.

Prova. Se $A_{0}$ é um aberto qualquer de $Y_{0}$, então existe um aberto $A$ de $Y$ tal que $A_{0}=A \cap Y$. Como $f^{-1}(A)$ é aberto em $X$ é óbvio que $g^{-1}\left(A_{0}\right)=f^{-1}(A) \cap X_{0}$ é aberto em $X_{0}$.

Obtemos a seguinte conseqüência da Proposição 3.3.5:

Corolário 3.3.7. Sejam $\Omega, V \subset \mathbb{C}^{n}$, abertos tais que $\emptyset \neq V \subset \Omega$. Então a restrição (que é homomorfismo de álgebras)

$$
{ }^{*} R_{V}^{\Omega}:\left.f \in\left(\mathcal{H G}_{f}(\Omega), \tau_{\Omega f}\right) \mapsto f\right|_{V} \in\left(\mathcal{H G}_{f}(V), \tau_{V f}\right)
$$

é contínua.

No Corolário 3.3.7 $\left(\mathcal{H G}_{f}(\Omega), \tau_{\Omega f}\right)$ denota $\mathcal{H} \mathcal{G}_{f}(\Omega)$ munida da topologia induzida $\tau_{\Omega f}$ sobre $\mathcal{G}_{f}(\Omega)$, a mesma cọisa para $\left(\mathcal{H G}_{f}(V), \tau_{V f}\right)$

Observemos por fim que vale um resultado bem mais geral que aquele da Observação 3.3.4, a saber:

Proposição 3.3.8. Se

$$
P=\sum_{|\alpha| \leq m} a_{\alpha}(x) \partial^{\alpha}
$$

é um operador diferencial parcial linear generalizado $O D P L G$ (isto é, $a_{\alpha} \in \mathcal{G}_{f}(\Omega), \forall|\alpha| \leq$ $m) e\left\{f_{l}\right\}_{l \in \mathbb{N}} \subset \mathcal{G}_{f}(\Omega)$ que é $\tau_{\Omega f}$-convergente a $f$, então $P\left(f_{l}\right) \underset{l \rightarrow \infty}{\longrightarrow} P(f)$. Em outros termos, $P$ é $\tau_{\Omega f}$-contínua.

Prova. A continuidade da multiplicação em $\mathcal{G}_{f}(\Omega)$ mostra que para cada $a \in \mathcal{G}_{f}(\Omega)$ a função $M_{a}: f \in \mathcal{G}_{f}(\Omega) \mapsto a f \in \mathcal{G}_{f}(\Omega)$ é $\tau_{\Omega f}$-contínua. Agora, pelo Corolário 3.3.2, 
sabemos que $\partial^{\alpha} f_{l} \underset{l \rightarrow \infty}{\longrightarrow} \partial^{\alpha} f$ e, portanto, $M_{a}\left(\partial^{\alpha} f_{l}\right) \underset{l \rightarrow \infty}{\longrightarrow} M_{a}\left(\partial^{\alpha} f\right)$, isto é, $a\left(\partial^{\alpha} f_{l}\right) \underset{l \rightarrow \infty}{\longrightarrow}$ $a\left(\partial^{\alpha} f\right)$. Assim, obviamente temos que $a_{\alpha}\left(\partial^{\alpha} f_{l}\right) \underset{l \rightarrow \infty}{\longrightarrow} a_{\alpha}\left(\partial^{\alpha} f\right), \forall|\alpha| \leq m$ e, portanto, pela continuidade da adição em $\mathcal{G}_{f}(\Omega)$ mostra que $P\left(f_{l}\right) \underset{l \rightarrow \infty}{\longrightarrow} P(f)$.

$\mathrm{O}$ resultado a seguir garante que as funções generalizadas com suporte compacto $\mathcal{G}_{f_{c}}(\Omega)$ é denso em $\mathcal{G}_{f}(\Omega)$.

Corolário 3.3.9. Sejam $\left\{\Omega_{\nu}\right\}_{\nu \in \mathbb{N}}$ uma seqüência exaustiva de abertos para $\Omega^{1}$ e $\mathcal{X}=$ $\left\{\mathcal{X}_{\nu}\right\}_{\nu \in \mathbb{N}}$ uma familia regularizante associada a $\left\{\Omega_{\nu}\right\}_{\nu \in \mathbb{N}}$ (isto é, $\mathcal{X}_{\nu} \in \mathcal{D}\left(\Omega_{\nu+1}\right)$ e $\left.\mathcal{X}_{\nu}\right|_{\Omega_{\nu}} \equiv$ $1, \forall \nu \in \mathbb{N}$ ), então $\mathcal{X}_{l} f \underset{l \rightarrow \infty}{\longrightarrow} f, \forall f \in \mathcal{G}_{f}(\Omega)$ e, em conseqüência $\mathcal{G}_{f_{c}}(\Omega)$ é denso em $\left(\mathcal{G}_{f}(\Omega), \tau_{\Omega f}\right)$.

Prova. Pela Proposição 3.3.1 precisamos mostrar que para toda sequêencia finita $s=$ $\left\{\left(m_{k}, p_{k}\right)\right\}_{1 \leq k \leq \nu} \subset \mathbb{N}^{2}$ e para todo $\left.a \in\right] 0,1\left[\right.$ existe $l_{0} \in \mathbb{N}$ tal que para todo $l \geq l_{0}$, existem $\varepsilon_{l} \in I$ e $N_{k} \in \mathbb{N}$ tal que se $0<\varepsilon<\varepsilon_{l}$ e $1 \leq k \leq \nu$, então

$$
\left\|\partial^{\beta}\left(\mathcal{X}_{l} f-f\right)\left(\varphi_{\varepsilon}, \cdot\right)\right\|_{m_{k}} \leq \varepsilon^{-\ln a}, \forall \varphi \in \mathcal{A}_{N_{k}}(\mathbb{K}), \forall|\beta| \leq p_{k} .
$$

De fato, se tomarmos $l_{0}=m_{k}$, então para todo $l \geq l_{0}=m_{k}$ temos que $\Omega_{m_{k}} \subset \Omega_{l}$ o que implica $\left.\mathcal{X}_{l}\right|_{\Omega_{m_{k}}} \equiv 1 \mathrm{e}$, portanto, $\left.\mathcal{X}_{l} f\right|_{\Omega_{m_{k}}}=\left.f\right|_{\Omega_{m_{k}}}, \forall l \geq l_{0}=m_{k} \mathrm{e}$, assim, $\left(\varepsilon_{l}=1 \mathrm{e}\right.$ $\left.N_{k}=0\right)$ temos que

$$
\left\|\partial^{\beta}\left(\mathcal{X}_{l} f-f\right)\left(\varphi_{\varepsilon}, \cdot\right)\right\|_{m_{k}}=0<\varepsilon^{-\ln a}, \forall|\beta| \leq p_{k}, \forall \varphi \in \mathcal{A}_{0}(\mathbb{K}) .
$$

Isto é,

$$
\left\|\partial^{\beta}\left(\mathcal{X}_{l} f-f\right)\left(\varphi_{\varepsilon}, \cdot\right)\right\|_{m_{k}}<\varepsilon^{-\ln a}, \forall|\beta| \leq p_{k}, \forall \varphi \in \mathcal{A}_{0}(\mathbb{K}) .
$$

que é exatamente a desigualdade em (3.30) e, portanto, segue o resultado.

\subsection{A topologia cortante sobre $\mathcal{G}_{f}(\bar{\Omega}), \Omega \subset \mathbb{R}^{n}$ aberto limitado}

Dedicamos esta seção aos seminários realizados com o Aragona, Orlando e eu, que culminou nos manuscritos [4] o qual define uma topologia cortante sobre $\mathcal{G}_{f}(\bar{\Omega})$, onde

\footnotetext{
${ }^{1}$ Isto é, $\Omega_{\nu}$ é aberto, $\bar{\Omega}_{\nu} \subset \subset \Omega_{\nu+1}, \forall \nu \in \mathbb{N}$ e $\Omega=\bigcup_{\nu \in \mathbb{N}} \Omega_{\nu}$
} 
$\Omega \subset \mathbb{R}^{n}$ é um aberto limitado e com esta topologia foi possível mostrar a completude de $\mathcal{G}_{f}(\bar{\Omega})$, onde $\Omega \subset \mathbb{R}^{n}$ aberto limitado. Como corolário deste resultado temos que a álgebra das funções generalizadas plenas de Colombeau sobre $\Omega, \mathcal{G}_{f}(\Omega)$ é completa. O objetivo de incluirmos em nosso trabalho estas notas é que vamos precisar delas para generalizar um problema de valor inicial e de fronteira devido a Colombeau e Langlais [10]. Naturalmente, fizemos adaptações para as nossas notações com o intuito de uniformizarmos a notação.

Suponhamos que $\Omega$ é um aberto, não vazio, limitado de $\mathbb{R}^{n}$. Dado $f \in \mathcal{G}_{f}(\bar{\Omega})$ qualquer, se $\hat{f} \in \mathcal{E}_{f}^{M}(\bar{\Omega})$ é um representante qualquer de $f$ e $\alpha \in \mathbb{N}^{n}$ é claro que

$$
\partial \hat{f}(\varphi, \cdot) \in \mathrm{C}^{\infty}(\bar{\Omega}), \forall \varphi \in \mathcal{A}_{0}(\mathbb{K})
$$

e, portanto,

$$
\left\|\partial^{\alpha} \hat{f}(\varphi, \cdot)\right\|:=\left\|\partial^{\alpha} \hat{f}(\varphi, \cdot)\right\|_{\bar{\Omega}}<+\infty, \forall \varphi \in \mathcal{A}_{0}(\mathbb{K})
$$

O lema seguinte associa a cada $u \in \mathcal{E}_{f}^{M}(\bar{\Omega})$ e $\alpha \in \mathbb{N}^{n}$, multíndice, um elemento bem definido $u_{\alpha}$ em $\mathcal{E}_{f}^{M}(\mathbb{R})$ o qual é sua classe $\operatorname{cl}\left[u_{\alpha}\right] \in \overline{\mathbb{R}}_{f_{+}}$. Mais precisamente, temos

Lema 3.4.1. Dado $u \in \mathcal{E}_{f}^{M}(\bar{\Omega})$ e $\beta \in \mathbb{N}^{n}$, temos:

(a) A função $u_{\beta}: \mathcal{A}_{0}(\mathbb{K}) \rightarrow \mathbb{R}_{+}$tal que $u_{\beta}(\varphi)=\left\|\partial^{\beta} u(\varphi, \cdot)\right\|_{\bar{\Omega}}$ é moderada (i.e. $u_{\beta} \in$ $\left.\mathcal{E}_{f}^{M}(\mathbb{R})\right) e \operatorname{cl}\left[u_{\beta}\right] \in \overline{\mathbb{R}}_{f_{+}} ;$

(b) se $v \in \mathcal{E}_{f}^{M}(\bar{\Omega})$ e $u-v \in \mathcal{N}_{f}(\bar{\Omega})$, então $u_{\beta}-v_{\beta} \in \mathcal{N}_{f}(\mathbb{R})$ e, portanto, $\operatorname{cl}\left[u_{\beta}\right]=\operatorname{cl}\left[v_{\beta}\right]$.

Prova. (a) De fato, como $u \in \mathcal{E}_{f}^{M}(\bar{\Omega})$ segue que associado a $\bar{\Omega} \subset \subset \bar{\Omega}$ e a $\beta \in \mathbb{N}^{n}$ existe $N \in \mathbb{N}$ tal que para todo $\varphi \in \mathcal{A}_{N}(\mathbb{K})$ existem $C=C(\varphi)>0$ e $\eta=\eta(\varphi)>\in I$ de modo que

$$
\left\|\partial^{\beta} u\left(\varphi_{\varepsilon}, \cdot\right)\right\| \leq c \varepsilon^{-N}, \forall \varepsilon \in I_{\eta}
$$

ou seja

$$
u_{\beta}\left(\varphi_{\varepsilon}\right)=\left|u_{\beta}\left(\varphi_{\varepsilon}\right)\right| \leq C \varepsilon^{-N}, \forall \varepsilon \in I_{\eta}
$$

Em resumo, mostramos que existe $N \in \mathbb{N}$ tal que para toda $\varphi \in \mathcal{A}_{N}(\mathbb{K})$ existem $C=$ $C(\varphi)>0$ e $\eta(\varphi)>0$ de modo que

$$
\left|u_{\beta}\left(\varphi_{\varepsilon}\right)\right| \leq C \varepsilon^{-N}, \forall \varepsilon \in I_{\eta} .
$$

e, portanto, $u_{\beta} \in \mathcal{E}_{f}^{M}(\mathbb{R})$. E, assim, $\operatorname{cl}\left[u_{\beta}\right] \in \overline{\mathbb{R}}_{f_{+}}$ 
(b) Como $u-v \in \mathcal{N}_{f}(\bar{\Omega})$, associados a $\bar{\Omega} \subset \subset \bar{\Omega}$ e a $\beta \in \mathbb{N}^{n}$, existem $N \in \mathbb{N}$ e $\gamma \in \Gamma$ tal que para todo $q \geq N$ e para toda $\varphi \in \mathcal{A}_{q}$ existem $C=C(\varphi)>0$ e $\eta=\eta(\varphi) \in I$ de modo que

$$
\left\|\partial^{\beta}(u-v)\left(\varphi_{\varepsilon}, \cdot\right)\right\| \leq C \varepsilon^{\gamma(q)-N}, \forall \varepsilon \in I_{\eta}
$$

Ora,

$$
\partial^{\beta}(u-v)(\varphi, \cdot)=\partial^{\beta} u(\varphi, \cdot)-\partial^{\beta} v(\varphi, \cdot), \forall \varphi \in \mathcal{A}_{0}(\mathbb{K})
$$

mas

$$
\left\|\partial^{\beta} u(\varphi, x)|-| \partial^{\beta} v(\varphi, x)\right\| \leq\left|\partial^{\beta} u(\varphi, x)-\partial^{\beta} v(\varphi, x)\right|, \forall \varphi \in \mathcal{A}_{0}(\mathbb{K}), \forall x \in \bar{\Omega}
$$

Por [3], Lema 3.1, temos que

$$
\left|\left\|\partial^{\beta} u(\varphi, \cdot)\right\|-\left\|\partial^{\beta} v(\varphi, \cdot)\right\|\right| \leq\left\|\partial^{\beta}(u-v)(\varphi, \cdot)\right\|, \forall \varphi \in \mathcal{A}_{0}(\mathbb{K})
$$

o que juntamente com (3.31) mostrar que para toda $\varphi \in \mathcal{A}_{q}(\mathbb{K})$ tem-se

$$
\left|\left\|\partial^{\beta} u(\varphi, \cdot)\right\|-\left\|\partial^{\beta} v(\varphi, \cdot)\right\|\right| \leq C \varepsilon^{\gamma(q)-N}, \forall \varepsilon \in I_{\eta} .
$$

Isto é,

$$
\left|u_{\beta}\left(\varphi_{\varepsilon}\right)-v_{\beta}\left(\varphi_{\varepsilon}\right)\right| \leq C \varepsilon^{\gamma(q)-N}, \forall \varepsilon \in I_{\eta} .
$$

E, portanto, $u_{\beta}-v_{\beta} \in \mathcal{N}_{f}(\mathbb{R})$ o que implica $\operatorname{cl}\left[u_{\beta}\right]=\operatorname{cl}\left[v_{\beta}\right] \in \overline{\mathbb{R}}_{f}$.

Do Lema 3.4.1 tem sentido a seguinte:

Definição 3.4.2. Fixado $\beta \in \mathbb{N}^{n}$ qualquer. Para todo $u \in \mathcal{G}_{f}(\bar{\Omega})$ definimos

$$
\|u\|_{\beta}:=\operatorname{cl}\left[\hat{u}_{\beta}\right]=\operatorname{cl}\left[\varphi \in \mathcal{A}_{0}(\mathbb{K}) \mapsto\left\|\partial^{\beta} \hat{u}(\varphi, \cdot)\right\|_{\Omega} \in \mathbb{R}_{+}\right]
$$

onde $\hat{u}$ é um representante qualquer de $u$. Para todo $u_{0} \in \mathcal{G}_{f}(\bar{\Omega})$ e $r \in \mathbb{R}$ definimos

$$
W_{\beta, r}\left[u_{0}\right]:=\left\{u \in \mathcal{G}_{f}(\bar{\Omega}) \mid\left\|u-u_{0}\right\|_{\sigma} \leq \dot{\alpha}_{r}, \forall \sigma \leq \beta\right\}
$$

$e$

$$
\mathcal{B}_{\bar{\Omega}}:=\left\{W_{\beta, r}[0] \mid \beta \in \mathbb{N}^{n} \text { e } r \in \mathbb{R}\right\}
$$

Quando $u \equiv 0$ na Definição 3.4.2 escrevemos simplesmente $W_{\beta, r}$ no lugar de $W_{\beta, r}[0]$. Agora, lembrando que $\overline{\mathbb{K}}_{f} \subset \mathcal{G}_{f}(\bar{\Omega})$ como o anel das constantes, temos o seguinte: 
Lema 3.4.3. Para todo $\beta \in \mathbb{N}^{n}$ e $r \in \mathbb{R}$, temos que $\overline{\mathbb{K}}_{f} \cap W_{\beta, r}=V_{r}$.

Prova. Ver [4].

Lema 3.4.4. Sejam $f, g \in \mathcal{G}_{f}(\bar{\Omega})$ ( $\Omega$ domínio limitado). Então

(a) $\|f+g\|_{\sigma} \leq\|f\|_{\sigma}+\|g\|_{\sigma}, \forall \sigma \in \mathbb{N}^{n}$;

(b) $\|f g\|_{\sigma} \leq \sum_{\gamma \leq \sigma}\left(\begin{array}{c}\sigma \\ \gamma\end{array}\right)\|f\|_{\gamma}\|g\|_{\sigma-\gamma}, \forall \sigma \in \mathbb{N}^{n}$.

Prova. (a) Pela Definição 3.4 .2 é claro que $\|f+g\|_{\sigma}=\operatorname{cl}[v]$, onde

$$
v: \varphi \in \mathcal{A}_{0}(\mathbb{K}) \mapsto\left\|\partial^{\sigma}(\hat{f}+\hat{g})(\varphi, \cdot)\right\| \in \mathbb{R}_{+} .
$$

Aqui, temos que $\hat{f}, \hat{g}$ são representantes quaisquer fixados de $f$ e $g$, respectivamente. Ora, pela desigualdade triangular, temos que

$$
\left\|\partial^{\sigma}(\hat{f}+\hat{g})(\varphi, \cdot)\right\| \leq\left\|\partial^{\sigma} \hat{f}(\varphi, \cdot)\right\|+\left\|\partial^{\sigma} \hat{g}(\varphi, \cdot)\right\|, \forall \varphi \in \mathcal{A}_{0}(\mathbb{K})
$$

Novamente, pela Definição 3.4.2, temos que

$$
\begin{aligned}
\|f\|_{\sigma}+\|g\|_{\sigma} & =\operatorname{cl}\left[\varphi \mapsto\left\|\partial^{\sigma} \hat{f}(\varphi, \cdot)\right\|\right]+\operatorname{cl}\left[\varphi \mapsto\left\|\partial^{\sigma} \hat{g}(\varphi, \cdot)\right\|\right] \\
& =\operatorname{cl}\left[\varphi \mapsto\left\|\partial^{\sigma} \hat{f}(\varphi, \cdot)\right\|+\left\|\partial^{\sigma} \hat{g}(\varphi, \cdot)\right\|\right] \\
& =\operatorname{cl}[u]
\end{aligned}
$$

onde

$$
u: \varphi \in \mathcal{A}_{0}(\mathbb{K}) \mapsto\left\|\partial^{\sigma} \hat{f}(\varphi, \cdot)\right\|+\left\|\partial^{\sigma} \hat{g}(\varphi, \cdot)\right\| \in \mathbb{R}_{+}
$$

É, claro que (3.32) mostra que $v(\varphi) \leq u(\varphi), \forall \varphi \in \mathcal{A}_{0}(\mathbb{K})$, i.e.,

$$
(u-v)(\varphi) \geq 0, \forall \varphi \in \mathcal{A}_{0}(\mathbb{K})
$$

Portanto ([3], Lema 2.1 (iii) mais a Definição 2.2) $\operatorname{cl}[v] \leq \operatorname{cl}[u]$, isto é, vale $(a)$.

(b) Embora um pouquinho mais longa, é inteiramente análoga a (a). De fato, pela Definição 3.4 .2 , temos que $\|f g\|_{\sigma}=\operatorname{cl}[v]$, onde

$$
v: \varphi \in \mathcal{A}_{0}(\mathbb{K}) \mapsto\left\|\partial^{\sigma}(\hat{f} \hat{g})(\varphi, \cdot)\right\| \in \mathbb{R}_{+} .
$$


Pela fórmula de Leibnitz e pela desigualdade triangular, temos que

$$
\left\|\partial^{\sigma}(\hat{f} \hat{g})(\varphi, \cdot)\right\| \leq \sum_{\beta \leq \sigma}\left(\begin{array}{l}
\sigma \\
\beta
\end{array}\right)\left\|\partial^{\beta} \hat{f}(\varphi, \cdot)\right\|\left\|\partial^{\sigma-\beta} \hat{g}(\varphi, \cdot)\right\|, \forall \varphi \in \mathcal{A}_{0}(\mathbb{K}) .
$$

Por outro lado, de novo pela Definição 3.4 .2 , temos que

$$
\|f\|_{\beta}=\operatorname{cl}\left[\varphi \in \mathcal{A}_{0}(\mathbb{K}) \mapsto \mid \partial^{\beta} \hat{f}(\varphi, \cdot) \| \in \mathbb{R}_{+}\right]
$$

e

$$
\|g\|_{\sigma-\beta}=\operatorname{cl}\left[\varphi \in \mathcal{A}_{0}(\mathbb{K}) \mapsto\left\|\partial^{\sigma-\beta} \hat{g}(\varphi, \cdot)\right\| \in \mathbb{R}_{+}\right]
$$

donde (para $\beta \leq \sigma)$, temos

$$
\begin{aligned}
\left(\begin{array}{c}
\sigma \\
\beta
\end{array}\right)\|f\|_{\beta}\|g\|_{\sigma-\beta} & =\left(\begin{array}{c}
\sigma \\
\beta
\end{array}\right) \operatorname{cl}\left[\varphi \mapsto\left\|\partial^{\beta} \hat{f}(\varphi, \cdot)\right\|\right] \operatorname{cl}\left[\varphi \mapsto\left\|\partial^{\sigma-\beta} \hat{g}(\varphi, \cdot)\right\|\right] \\
& =\operatorname{cl}\left[\varphi \mapsto\left(\begin{array}{l}
\sigma \\
\beta
\end{array}\right)\left\|\partial^{\beta} \hat{f}(\varphi, \cdot)\right\|\left\|\partial^{\sigma-\beta} \hat{g}(\varphi, \cdot)\right\|\right] .
\end{aligned}
$$

E, portanto,

$$
\begin{aligned}
\sum_{\beta \leq \sigma}\left(\begin{array}{l}
\sigma \\
\beta
\end{array}\right)\|f\|_{\beta}\|g\|_{\sigma-\beta} & =\operatorname{cl}\left[\varphi \mapsto \sum_{\beta \leq \sigma}\left(\begin{array}{l}
\sigma \\
\beta
\end{array}\right)\left\|\partial^{\beta} \hat{f}(\varphi, \cdot)\right\|\left\|\partial^{\sigma-\beta} \hat{g}(\varphi, \cdot)\right\|\right] \\
& =\operatorname{cl}[u]
\end{aligned}
$$

onde

$$
u: \varphi \in \mathcal{A}_{0}(\mathbb{K}) \mapsto \sum_{\beta \leq \sigma}\left(\begin{array}{l}
\sigma \\
\beta
\end{array}\right)\left\|\partial^{\beta} \hat{f}(\varphi, \cdot)\right\|\left\|\partial^{\sigma-\beta} \hat{g}(\varphi, \cdot)\right\| \in \mathbb{R}_{+} .
$$

É claro que (3.33) expressa que $v(\varphi) \leq u(\varphi), \forall \varphi \in \mathcal{A}_{0}(\mathbb{K})$, i.e.,

$$
(u-v)(\varphi) \geq 0, \forall \varphi \in \mathcal{A}_{0}(\mathbb{K})
$$

Portanto ([3], Lema 2.1 (iii) mais Definição 2.2) resulta que cl[ $v] \leq \mathrm{cl}[u]$ que é exatamente (b).

Lema 3.4.5 ( $\alpha$-lema). Quaisquer que sejam $k, r \in \mathbb{R}_{+}^{*}$ tem-se que $k\left(\dot{\alpha}_{r}\right)^{2} \leq \dot{\alpha}_{r}$.

Prova. Tomemos $N=0$, fixemos $b>0$ e $\varphi \in \mathcal{A}_{N}(\mathbb{K})=\mathcal{A}_{0}(\mathbb{K})$ arbitrários, devemos então achar $\eta=\eta(b, \varphi) \in I$ tal que

$$
\left(\hat{\dot{\alpha}}_{r}-k\left(\hat{\dot{\alpha}}_{r}\right)^{2}\right)\left(\varphi_{\varepsilon}\right)>-\varepsilon^{b}, \forall \varepsilon \in I_{\eta} .
$$


Como $\hat{\dot{\alpha}}_{r}\left(\varphi_{\varepsilon}\right)=\varepsilon^{r}(i(\varphi))^{r}$ e $k\left(\hat{\dot{\alpha}}_{r}\right)^{2}\left(\varphi_{\varepsilon}\right)=k \varepsilon^{2 r}(i(\varphi))^{2 r}$, o primeiro membro de (3.34) se escreve assim:

$$
T=T\left(\varphi_{\varepsilon}\right):=\varepsilon^{r}(i(\varphi))^{r}-k \varepsilon^{2 r}(i(\varphi))^{2 r}
$$

ou seja

$$
T=\varepsilon^{r}(i(\varphi))^{r}\left(1-k \varepsilon^{r}(i(\varphi))^{r}\right)
$$

e, portanto, basta que $T \geq 0$ e para tanto é suficiente que $1-k \varepsilon^{r}(i(\varphi))^{r} \geq 0$, isto é, $\left.k \varepsilon^{r}(i(\varphi))^{r}\right) \leq 1$ ou ainda $\varepsilon^{r} \leq \frac{1}{k(i(\varphi))^{r}}$, isto é, $\varepsilon \leq \eta:=\frac{1}{i(\varphi) k^{\frac{1}{r}}}$. Em resumo, definindo

$$
\eta=\eta(b, \varphi):=\frac{1}{i(\varphi) k^{\frac{1}{r}}}
$$

temos

$$
T\left(\varphi_{\varepsilon}\right)=\left(\hat{\dot{\alpha}}_{r}-k\left(\hat{\dot{\alpha}}_{r}\right)^{2}\right)\left(\varphi_{\varepsilon}\right) \geq 0>-\varepsilon^{-b}, \forall \varepsilon \in I_{\eta}
$$

E, portanto, segue nossa assertiva.

Corolário 3.4.6. Para todo $k, r \in \mathbb{R}_{+}^{*}$ tem-se que $k \dot{\alpha}_{s} \leq \dot{\alpha}_{r}, \forall s \geq r$.

Prova. Com efeito, se $s>r$, então $\dot{\alpha}_{s} \leq \dot{\alpha}_{r}$ (óbvio) $\therefore \dot{\alpha}_{s}^{2} \leq \dot{\alpha}_{r}^{2} \therefore k \dot{\alpha}_{s}^{2} \leq k \dot{\alpha}_{r}^{2} \leq \dot{\alpha}_{r}$. Esta última desigualdade segue do Lema 3.4.5.

Podemos finalmente mostrar que a multiplicação em $\mathcal{G}_{f}(\bar{\Omega})$ é contínua $(0,0)$, isto é, dado $W_{\alpha, r} \in \mathcal{B}_{\bar{\Omega}, b}$ arbitrário existe $W_{\beta, s} \in \mathcal{B}_{\bar{\Omega}, b}$ tal que $W_{\beta, s}^{2} \subset W_{\alpha, r}$. Na realidade, em vista do enunciado do $\alpha$-lema acima, o que vamos mostrar é o seguinte:

Lema 3.4.7 $\left(A V_{I I}^{\prime}\right)$. Para todo $W_{\alpha, r} \in \mathcal{B}_{\bar{\Omega}, b}$ tem-se que $W_{\alpha, r}^{2} \subset W_{\alpha, r}$

Prova. Sejam

$$
\begin{gathered}
M_{\sigma}:=\max _{\beta \leq \sigma}\left\{\left(\begin{array}{c}
\sigma \\
\beta
\end{array}\right)\right\}, M:=\max _{\sigma \leq \alpha}\left\{M_{\sigma}\right\}, \\
p_{\sigma}:=\text { o número de somando em } \sum_{\beta \leq \sigma}\left\{\left(\begin{array}{l}
\sigma \\
\beta
\end{array}\right)\right\}, p:=\max _{\sigma \leq \alpha}\left\{p_{\sigma}\right\} \text { e } k:=M_{p} .
\end{gathered}
$$

Então, devemos mostrar que

$$
f, g \in W_{\alpha, r} \Rightarrow f g \in W_{\alpha, r}
$$

De fato,

$$
f, g \in W_{\alpha, r} \Leftrightarrow\|f\|_{\lambda} \leq \dot{\alpha}_{r}, \text { e }\|g\|_{\lambda} \leq \dot{\alpha}_{r}, \forall \lambda \leq \alpha .
$$


Portanto, se

$$
\|f\|_{*}:=\max _{\lambda \leq \alpha}\left\{\|f\|_{\lambda}\right\} \text { e }\|g\|_{*}:=\max _{\lambda \leq \alpha}\left\{\|g\|_{\lambda}\right\}
$$

resulta por (3.36):

$$
\|f\|_{*} \leq \dot{\alpha}_{r} \text { e }\|g\|_{*} \leq \dot{\alpha}_{r}
$$

Então pelo Lema 3.4.4 (b) resulta, para cada $\sigma \leq \alpha$ :

$$
\begin{aligned}
\|f g\|_{\sigma} & \leq \sum_{\beta \leq \sigma}\left(\begin{array}{l}
\sigma \\
\beta
\end{array}\right)\|f\|_{\beta}\|g\|_{\sigma-\beta} \\
& \leq p_{\sigma} M_{\sigma}\|f\|_{*}\left\|_{g}\right\|_{*} \\
& \leq p M\|f\|_{*}\|g\|_{*} \\
& \leq k\left(\dot{\alpha}_{r}\right)^{2} \\
& \leq \dot{\alpha}_{r} .
\end{aligned}
$$

Isto é, $\|f g\|_{\sigma} \leq \dot{\alpha}_{r}$ o que mostra (3.35).

Para mostrarmos que a topologia $\tau_{\bar{\Omega}, b}$ é compatível com a estrutura de $\overline{\mathbb{K}}_{f}$-álgebra de $\mathcal{G}_{f}(\bar{\Omega})$ (já mostramos que $\tau_{\bar{\Omega}, b}$ é compatível com a estrutura de grupo abeliano de $\mathcal{G}_{f}(\bar{\Omega})$ ), como acabamos de verificar $A V_{I I}^{\prime}$ (no Lema 3.4.7), em vista de ([3], Corolário 1.3) resta apenas verificar a validade de:

$\left(A V_{I}^{\prime}\right)$ Fixados $g \in \mathcal{G}_{f}(\bar{\Omega})$ e $W_{\alpha, r} \in \mathcal{B}_{\bar{\Omega}, b}$ arbitrário existe $W_{\beta, s} \in \mathcal{B}_{\bar{\Omega}, b}$ tal que $g W_{\beta, s} \subset$ $W_{\alpha, r}$ (isto é, a homotetia $f \mapsto f g$ é contínua em 0 ).

Isto segue facilmente dos dois lemas seguintes:

Lema 3.4.8. Fixados $g \in \mathcal{G}_{f}(\bar{\Omega})$ e $\alpha \in \mathbb{N}^{m}$ quaisquer, existem $C>0$ e $t \in \mathbb{R}$ (provavelmente $t<0$ ) tal que $\|g\|_{\sigma} \leq C \dot{\alpha}_{t}, \forall \sigma \leq \alpha$

Prova. Seja $\hat{g} \in \mathcal{E}_{f}^{M}(\bar{\Omega})$ um representante qualquer de $g$ então, associado a $\alpha \in \mathbb{N}^{m}$ (e a $\bar{\Omega} \subset \subset \bar{\Omega})$ existe $N \in \mathbb{N}$ tal que para toda $\varphi \in \mathcal{A}_{N}(\mathbb{K})$ existem $c>0$ e $\eta \in I$ de modo que

$$
\left\|\partial^{\sigma} \hat{g}\left(\varphi_{\varepsilon}, \cdot\right)\right\| \leq c \varepsilon^{-N}, \forall \varepsilon \in I_{\eta}, \forall \sigma \leq \alpha .^{2}
$$

Tomando $C:=c(i(\varphi))^{N}$ podemos escrever a desigualdade acima como segue:

$$
\left\|\partial^{\sigma} \hat{g}\left(\varphi_{\varepsilon}, \cdot\right)\right\| \leq C \hat{\dot{\alpha}}_{-N}\left(\varphi_{\varepsilon}\right), \forall \varepsilon \in I_{\eta}, \forall \sigma \leq \alpha
$$

\footnotetext{
${ }^{2} \mathrm{Na}$ realidade, a condição de moderação vale apenas para $\sigma$, mas visto que o conjunto dos $\sigma \leq \alpha$ é finito, é óbvio que a coisa toda vale para todo $\sigma \leq \alpha$.
} 
E, portanto,

$$
\|g\|_{\sigma} \leq C \dot{\alpha}_{-N}, \forall \sigma \leq \alpha
$$

(o que mostra o Lema 3.4.8 definindo $t:=-N$ ).

Podemos enunciar melhor o Lema 3.4.8 assim:

Lema 3.4.9. Fixados $g \in \mathcal{G}_{f}(\bar{\Omega})$ e $\alpha \in \mathbb{N}^{m}$ arbitrários existem $C>0$ e $N \in \mathbb{N}$ tais que $\|g\|_{\sigma} \leq C \dot{\alpha}_{-N}, \forall \sigma \leq \alpha$.

Lema 3.4.10. Dados $k>0, r>0$ e $N_{1} \in \mathbb{N}$ quaisquer existe $s>0$ tal que $k \dot{\alpha}_{-N_{1}} \dot{\alpha}_{s} \leq \dot{\alpha}_{r}$. De modo mais preciso, basta tomarmos $s \geq N_{1}+r+1$, isto é, $k \dot{\alpha}_{-N_{1}} \dot{\alpha}_{N_{1}+r+1} \leq \dot{\alpha}_{r}$.

Prova. Fixados os representantes arbitrários $\hat{\dot{\alpha}}_{l}\left(l=-N_{1}, s, r\right)$ tomemos $N=0$ e fixemos $b>0$ e $\varphi \in \mathcal{A}_{N}(\mathbb{K})=\mathcal{A}_{0}(\mathbb{K})$ arbitrários, então

$$
\begin{aligned}
\left(\hat{\dot{\alpha}}_{r}-k \hat{\dot{\alpha}}_{-N_{1}} \hat{\dot{\alpha}}_{N_{1}+r+1}\right)\left(\varphi_{\varepsilon}\right) & =\hat{\dot{\alpha}}_{r}\left(\varphi_{\varepsilon}\right)-k \hat{\dot{\alpha}}_{-N_{1}}\left(\varphi_{\varepsilon}\right) \hat{\dot{\alpha}}_{N_{1}+r+1}\left(\varphi_{\varepsilon}\right) \\
& =\varepsilon^{r}(i(\varphi))^{r}-k \varepsilon^{-N_{1}}\left(i(\varphi)^{-N_{1}} \varepsilon^{N_{1}+r+1}(i(\varphi))^{N_{1}+r+1}\right. \\
& =\varepsilon^{r}(i(\varphi))^{r}(1-k i(\varphi) \varepsilon)>0 \geq-\varepsilon^{b}
\end{aligned}
$$

para todo $\varepsilon>0$ tal que $1-k i(\varphi) \varepsilon>0$, ou seja, $\varepsilon<\eta:=\frac{1}{k} i(\varphi)$. Portanto,

$$
\left(\hat{\dot{\alpha}}_{r}-k \hat{\dot{\alpha}}_{-N_{1}} \hat{\dot{\alpha}}_{N_{1}+r+1}\right)\left(\varphi_{\varepsilon}\right)>-\varepsilon^{b}, \forall \varepsilon<\eta=\eta(b, \varphi):=\frac{1}{k i(\varphi)} .
$$

Segue assim o resultado.

Vamos agora mostrar a afirmação $\left(A V_{I}^{\prime}\right)$ :

Prova. Partimos dos seguintes dados: $W_{\alpha, r}$ e $g$. Pelo Lema 3.4.9, associados a $g$ e a $\alpha$ fica determinado $N \in \mathbb{N}$ tal que

$$
\|g\|_{\sigma} \leq C \dot{\alpha}_{-N}, \forall \sigma \leq \alpha
$$

Sejam $M, p$ (e $\left.M_{\sigma}, p_{\sigma}\right)$ como na prova do Lema 3.4.7 $\left(A V_{I I}^{\prime}\right)$ e $k:=M p C$ (onde $C$ aparece em (3.37) acima graças ao Lema 3.4.9). Associados a $k, r$ (de $W_{\alpha, r}$ ) e $N \in \mathbb{N}$ (que também aparece em (3.37) acima graças ao Lema 3.4.9), pelo Lema 3.4.10, existe $s:=N+r+1$ tal que

$$
k \dot{\alpha}_{-N} \dot{\alpha}_{N+r+1} \leq \dot{\alpha}_{r}
$$


Definimos então $W_{\beta, s} \operatorname{com} \beta:=\alpha$ e $s:=N+r+1$ e vamos mostrar que

$$
g W_{\alpha, N+r+1} \subset W_{\alpha, r} .
$$

De fato, fixado $f \in W_{\alpha, N+r+1}$, isto é, $\|f\|_{\sigma} \leq \dot{\alpha}_{N+r+1}, \forall \sigma \leq \alpha$ resulta (Lema 3.4.4, (b)) por (3.37) e (3.38):

$$
\begin{aligned}
\|g f\|_{\sigma} & \leq \sum_{\beta \leq \sigma}\left(\begin{array}{l}
\sigma \\
\beta
\end{array}\right)\|g\|_{\sigma}\|f\|_{\sigma-\beta} . \\
& \leq M p\left(C \dot{\alpha}_{-N}\right) \dot{\alpha}_{N+r+1} \\
& \leq \dot{\alpha}_{r} .
\end{aligned}
$$

E, portanto, $\|g f\|_{\sigma} \leq \dot{\alpha}_{r} . \forall \sigma \leq \alpha$ o que implica $g f \in W_{\alpha, r}$ o que mostra (3.39).

Para finalizarmos esta seção indicamos os manuscritos do Aragona intitulado: "A completude das álgebras de Colombeau $\mathcal{G}_{f}(\Omega)$ e $\mathcal{G}_{f}(\bar{O})$, onde $\Omega$ e $O$ são abertos de $\mathbb{R}^{n}$ e $O$ é limitado." (Ver [4].) Nestes manuscritos o professor definiu uma topologia cortante, que denotou por $\tau_{\bar{O}, b}$, para $\mathcal{G}_{f}(\bar{O})$ e com esta topologia mostrou que este espaço é completo e como corolário mostrou também que $\mathcal{G}_{f}(\Omega)$ é completo. Vamos então apresentar os resultados de que vamos usar em nosso trabalho. Iniciamos com o seguinte:

Lema 3.4.11. Fixados $\sigma \in \mathbb{N}^{m}$ e $r \in \mathbb{N}^{*}$ arbitrários, para $g \in \mathcal{E}_{f}^{M}(\bar{O})$ são equivalentes as duas condições seguintes:

(i) $g \in W_{\sigma, r}$;

(ii) para todo representante $\hat{g} \in \mathcal{E}_{f}^{M}(\bar{O})$ de $g$ (e o representante $\hat{\dot{\alpha}}_{r}$ de $\dot{\alpha}_{r}$ ) vale a condição: Existe um $N \in \mathbb{N}$ tal que para todo $b>0$ e para toda $\varphi \in \mathcal{A}_{N}(\mathbb{K})$ existe $\eta=\eta(b, \varphi) \in I$ verificando

$$
\left\|\partial^{\beta} \hat{g}\left(\varphi_{\varepsilon}, \cdot\right)\right\| \leq i(\varphi)^{r}+\varepsilon^{b}, \forall \varepsilon \in I_{\eta} e \forall \beta \leq \sigma .
$$

Prova. Ver [4].

Lema 3.4.12. Seja $\left\{f_{m}\right\}_{n \in \mathbb{N}}$ uma seqüência de Cauchy em $\left(\mathcal{G}_{f}(\bar{O}), \tau_{\bar{O}, b}\right)$ e fixemos uma seqüência $\left\{\hat{f}_{m}\right\}_{m \in \mathbb{N}}$ de representantes das $f_{m}$. Então:

(a) Existe duas seqüências estritamente crescentes

$$
\lambda: r \in \mathbb{N}^{*} \mapsto \nu_{r} \in \mathbb{N}^{*} \text { e } \mu: \nu_{r} \in \Im m(\lambda) \mapsto N_{\nu_{r}} \in \mathbb{N}^{*}
$$


tais que: $\forall r \in \mathbb{N}^{*}, \forall b>0 e \forall \varphi \in \mathcal{A}_{N_{\nu_{r}}} \exists \eta_{\nu_{r}}=\eta_{\nu_{r}}(b, \varphi) \in I$ verificando

$$
\left.\left.\| \hat{f}_{\nu_{i+1}}-\hat{f}_{\nu_{i}}\right)\left(\varphi_{\varepsilon}, \cdot\right)\right) \| \leq i(\varphi)^{r} \varepsilon^{r}+\varepsilon^{b}, \forall \varepsilon \in I_{\eta_{\nu_{r}}}
$$

onde $\forall l \in \mathbb{N}^{*}, \forall b>0 e \forall \varphi \in \mathcal{A}_{N_{\nu_{l}}}$ tem-se

$$
\eta_{\nu_{1}}(b, \varphi)>\eta_{\nu_{2}}(b, \varphi)>\ldots \eta_{\nu_{l}}(b, \varphi)>0
$$

(b) Vale a seguinte afirmação: $\forall \sigma \in \mathbb{N}^{m} e \forall r \in \mathbb{N}^{*} \exists k \in \mathbb{N}^{*}$ com $k \geq r$ tal que, $\forall l \in \mathbb{N}^{*}$ com $l \geq k$ fixado, $\exists p \in \mathbb{N}^{*}$ com $p \geq k+r$ tal que $\forall b>0$ e $\forall \varphi \in$ $\mathcal{A}_{N_{\nu_{p}}} \exists \eta^{*}=\eta^{*}(b, \varphi) \in I$, com $\eta^{*}(b, \varphi)<\eta_{\nu_{p}}(b, \varphi)$, de modo que

$$
\left\|\partial^{\beta}\left(\hat{f}_{\nu_{i+1}}-\hat{f}_{\nu_{i}}\right)\left(\varphi_{\varepsilon}, \cdot\right)\right\| \leq i(\varphi)^{r} \varepsilon^{r}+\varepsilon^{r}+\varepsilon^{b}, \forall \varepsilon \in I_{\eta^{*}} e \forall \beta \leq \sigma,
$$

sempre que $k \leq i \leq l$.

Prova. Ver [4].

Finalmente, vamos enunciar o resultado que garante que $\left(\mathcal{G}_{f}(\bar{O}), \tau_{\bar{O}, b}\right)$ é completo, a saber:

Teorema 3.4.13. Se $O$ é um aberto limitado de $\mathbb{R}^{m}$, então $\mathcal{G}_{f}(\bar{O})$ munido da topologia $\tau_{\bar{O}, b}$ é completa.

Prova. Ver [4].

Como corolário deste resultado, temos que $\left(\mathcal{G}_{f}\left(\Omega, \tau_{\Omega f}\right)\right.$ é completa. Mais precisamente, temos

Corolário 3.4.14. $\mathcal{G}_{f}(\Omega)$ munida da topologia $\tau_{\Omega f}$ é completa.

Prova. Ver [4]

Devido ao fato que o Teorema 3.4.13 e seu Corolário 3.4.14 já estão sendo escritos no formato de artigo, não colocamos suas provas, apenas indicamos o manuscrito que foi, como já havíamos dito antes, fruto dos seminários realizados por Aragona, Orlando e eu. Estes resultados foram fundamentais para resolver o problema de valor inicial e de fronteira (PVIF) do tipo parabólico originado no artigo do Brezis e Friedman (1983) [8], continuado com Colombeau e Langlais (1990) [10] e finalizado em 2006 no Capítulo 4, Seção 4.3 do nosso trabalho. (Ver Teorema 4.3.1.) 
A topologia cortante sobre a álgebra das funções generalizadas plenas de 88 Colombeau 
CAPÍTUlo 4

\section{Solução generalizada para um problema de valor inicial e de fronteira do tipo parabólico}

Neste capítulo vamos generalizar um resultado de existência e unicidade para um problema de valor inicial e de fronteira, abreviadamente PVIF, para uma equação parabólica devido a Colombeau e Langlais [10]. No referido trabalho Colombeau e Langlais mostraram que se o dado inicial é uma função generalizada com suporte compacto, i.e. $u_{0} \in \mathcal{G}_{f_{c}}(\Omega)$ então o PVIF para a equação parabólica $u_{t}-\Delta u+u^{3}=0$, num domínio regular limitado, admite uma única solução generalizada ${ }^{1}$. Então nosso propósito é mostrar (usando resultados da topologia cortante, Capítulo 3) que o mesmo permanece valendo sem que o dado inicial seja, necessariamente; uma função generalizada com suporte compacto. Para tanto vamos enunciar na Seção 4.2, sem demonstração, alguns resultados de [10], os quais vamos precisar no decorrer deste capítulo. É importante salientar que, igualmente ao trabalho de [10], toda função generalizada é a valores em $\mathbb{R}$.

\subsection{Desenvolvimento histórico do problema}

Analogamente ao que acontece com as equações algébricas, acontece com as equações diferenciais parciais. No seguinte sentido; uma equação algébrica aparentemente simples

\footnotetext{
${ }^{1}$ Solução no sentido das funções generalizadas plenas de Colombeau.
} 
pode não ter solução em um dado conjunto de números, mas quando tomamos uma extensão, conveniente, do conjunto dado a mesma equação algébrica passa a admitir uma solução no conjunto "maior" (maior no sentido da inclusão de conjuntos). Assim, também acontece com as equações diferencias parciais; uma equação diferencial parcial aparentemente simples pode não ter soluções em um dado conjunto de funções, mas se consideramos um outro conjunto de funções que contenha aquele conjunto dado, a mesma equação pode admitir soluções. Para uma equação diferencial parcial conhecemos os seguintes tipos de soluções: soluções clássicas (no sentido das funções clássicas), soluções fraca (no sentido das distribuições) e as soluções generalizadas (que são as soluções no sentido das funções generalizadas de Colombeau).

Como podemos ver, quanto maior o conjunto de números maiores são as chances de se obter uma solução para as equações algébricas e de modo análogo quanto maior for o conjunto das funções maiores são as chances de se obter soluções para uma equação diferencial parcial.

Quando estendemos um dado conjunto de números ou funções fazemos isto de tal modo que o conjunto maior absorva o máximo das propriedades do conjunto menor (ou conjunto anterir). Isto é para minimizarmos os prejuízos ao passarmos de um nível para outro. O problema é que, quando estendemos um dado conjunto, precisamos pagar um preço por isto e este preço é muitas vezes calculado no número de propriedades que perdemos ao estender o conjunto dado. Por exemplo ao passarmos de $\mathbb{R}$ para $\mathbb{C}$ perdemos uma propriedade importante dos números reais que é a ordem, um outro exemplo é que quando saímos do conjunto das funções clássicas para as distribuições $\mathcal{D}^{\prime}$ perdemos a multiplicação de funções. A álgebra das funções generalizadas de Colombeau que, surgiu neste contexto, é uma álgebra comutativa associativa satisfazendo as regras de Leibnitz que contém as funções clássicas e as distribuições e, neste conjunto está bem definida uma multiplicação.

Vejamos um paralelo de inclusões de conjuntos de números e funções:

$$
\left\{\begin{array}{l}
\mathbb{N} \subset \mathbb{Z} \subset \mathbb{Q} \subset \mathbb{R} \subset \mathbb{C} \\
\mathrm{C}^{\infty} \subset \cdots \subset \mathrm{C}^{k} \subset \cdots \subset \mathrm{C}^{0} \subset L_{\text {loc }}^{p} \subset \mathcal{D}^{\prime} \subset \mathcal{G}_{f} .
\end{array}\right.
$$

Pelas nossas discussões acima as equações algébricas têm mais chances de terem soluções em $\mathbb{C}$, conjunto dos números complexos e, as equações diferencias parciais têm mais chances de terem soluções em $\mathcal{G}_{f}$, a álgebra das funções generalizadas plenas de Colombeau. 
Em 1983 Brezis e Friedman [8] consideraram o problema de Cauchy para uma equação parabólica não-linear envolvendo medida como dado inicial (e uma condição de fronteira), a saber:

$$
\begin{cases}u_{t}-\Delta u+|u|^{p-1} u=0, & \text { sobre } Q=\Omega \times] 0, T[ \\ u(x, 0)=\delta(x), & \text { sobre } \Omega,\end{cases}
$$

onde $\Omega \subset \mathbb{R}^{n}$ é um domínio contendo a origem, $0<p<\infty, 0<T<\infty$ e $\delta(x)$ a massa de Dirac na origem. Então, mostraram que uma solução de (4.1) existe se, e somente se, $0<p<(n+2) / n$. Em particular, a equação:

$$
\begin{cases}u_{t}-\Delta u+u^{3}=0, & \text { sobre } Q=\Omega \times] 0, T[ \\ u(x, 0)=\delta(x), & \text { sobre } \Omega\end{cases}
$$

não tem solução para toda dimensão $n \geq 1$. Solução fraca em $Q$ : não existe $u \in L_{\text {loc }}^{p}(Q)$ (para $p \geq 1$ ) satisfazendo

$$
\left.u_{t}-\Delta u+u^{3}=0, \text { sobre } Q=\Omega \times\right] 0, T[,
$$

i.e.,

$$
-\iint u \varphi \mathrm{d} x \mathrm{~d} t-\iint u \Delta \varphi \mathrm{d} x \mathrm{~d} t+\iint u^{3} \varphi \mathrm{d} x \mathrm{~d} t=0, \forall \varphi \in \mathcal{D}(Q)
$$

e

$$
u(x, 0)=\delta(x), \text { em } \Omega
$$

no sentido: para toda função contínua $\varphi$ com suporte compacto em $\Omega$

$$
\text { ess } \lim _{t \rightarrow 0} \int_{\Omega} u(x, t) \varphi(x) \mathrm{d} x=\varphi(0) .
$$

Mais ainda, se aproximarmos $\delta$ por uma seqüência $\left\{\delta_{n}\right\}_{n \in \mathbb{N}}$ de funções suaves observamos que as soluções $u_{n}$ de

$$
\begin{cases}u_{t}-\Delta u+u^{3}=0, & \text { sobre } Q=\Omega \times] 0, T[ \\ u(x, t)=0, & \text { sobre } \partial \Omega \times] 0, T[\end{cases}
$$

com condição inicial

$$
u_{n}(x, 0)=\delta_{n}(x) \text { sobre } \Omega
$$

convergem uniformente à função identicamente nula sobre $\bar{Q}_{\eta}:=\bar{\Omega} \times[\eta, T]$, para todo $\eta>0$. E, portanto, existe um fenômeno chamado lei de fronteira em $t=0$ cujo resultado é uma perda da condição inicial. (Ver $[10,8]$.) 
A perda acima aparece como um problema de definição matemática; a condição (4.5) é também um ótimo caminho para expressar (4.4), pois no interior de $Q$ a solução $u$, limite das soluções $u_{n}$ é a função nula, a qual sua restrição a $t=0$ é a massa de Dirac na origem de $\Omega$. Esta situação lembra a situação da "função Delta de Dirac" antes ser entendida como uma medida.

Em 1990, sete anos depois que Brezis e Friedman mostraram em [8], que (4.2) não tem solução fraca (no sentido de distribuições) Colombeau e Langlais escreveram um artigo (ver [10]) cuja proposta era mostrar que a teoria das funções generalizadas em [7, 9, 11, 13] tem uma definição natural para se obter a solução de (4.2) com a condição de fronteira

$$
u(x, t)=0, \text { sobre } \partial \Omega \times[0, T]
$$

Então, usando técnicas clássicas sobre estas equações parabólicas, obtiveram teoremas de existência e unicidade de soluções no sentido das funções generalizadas plenas de Colombeau. Estes teoremas, enunciamos em nosso trabalho sem nos preocuparmos com sua prova, já que serão úteis ao nosso propósito. Nosso objetivo é, portanto, usar os resultados topológicos obtidos no Capítulo 3, a saber: a densidade de $\mathcal{G}_{f_{c}}(\Omega)$ em $\mathcal{G}_{f}(\Omega)$ e a completude de $\mathcal{G}_{f}(\bar{\Omega})$ (ver [4]) para generalizar os resultados de existência e unicidade de Colombeau e Langlais [10] completando assim, mais uma fase de desenvolvimento do problema iniciado por Brezis e Friedman [8]. A nossa generalização consiste no fato de como tomamos o dado inicial do problema; enquanto o dado inicial de Colombeau e Langlais [10] era função generalizada com suporte compacto o dado inicial que tomamos é qualquer função em $\mathcal{G}_{f}(\Omega)$.

\subsection{A solução generalizada dada por Colombeau e Langlais}

A base desta seção são os resultados obtidos por Colombeau e Langlais em [10]. Iniciamos pelo seguinte:

Lema 4.2.1 (Ver [10]). Para todo inteiro $k$ existe um polinômio $P_{k} \in \mathcal{P}[X]$ tal que se 
$u_{0} \in \mathcal{D}(\Omega)$ então a solução $u$ de

$$
\begin{cases}u_{t}-\Delta u+u^{3}=0, & \text { em } \bar{Q}=\bar{\Omega} \times[0, T], T>0 \\ u(x, 0)=u_{0}(x), & \text { em } \Omega \\ u(x, t)=0 & \text { sobre } \partial \Omega \times[0, T]\end{cases}
$$

satisfaz:

$$
\|u\|_{\mathrm{C}^{k}(\bar{Q})} \leq P_{k}\left(\left\|u_{0}\right\|_{\mathrm{C}^{2 k+1}(\bar{\Omega})}\right)
$$

O resultado acima será importante para mostrarmos que a seqüência $\left\{\hat{u}_{n}\right\}_{n \in \mathbb{N}} \subset \mathcal{G}_{f}(\bar{Q})$ de soluções do P.V.I.F. (4.9) é limitada. Esta seqüência de soluções é obtida a partir da seqüência de dados iniciais $\left\{\hat{u}_{0 n}\right\}_{n \in \mathbb{N}} \subset \mathcal{G}_{f_{c}}(\Omega)$ pelo seguinte:

Teorema 4.2 .2 (Ver $[10])$. Para toda $u_{0} \in \mathcal{G}_{f}(\Omega)$ a valores reais com suporte compacto existe $u \in \mathcal{G}_{f}(\bar{Q})$ solução de

$$
\begin{cases}u_{t}-\Delta u+u^{3}=0, & \text { em } \mathcal{G}_{f}(\bar{Q}) \\ \left.u\right|_{\bar{\Omega} \times\{0\}}=u_{0}, & \text { em } \mathcal{G}_{f}(\bar{\Omega}) \\ \left.u\right|_{\partial \Omega \times[0, T]}=0, & \text { em } \mathcal{G}_{f}(\partial \Omega \times[0, T])\end{cases}
$$

O Lema que segue usamos para mostrar que a seqüência $\left\{\hat{u}_{n}\right\}_{n \in \mathbb{N}} \subset \mathcal{G}_{f}(\bar{Q})$ é uma seqüência de Cauchy em $\mathcal{G}_{f}(\bar{Q})$. E usando que $\mathcal{G}_{f}(\bar{Q})$ é completa (ver [4]) provamos que tal seqüência converge para a solução de (4.9).

Lema 4.2.3 (Ver [10]). Seja $v \in \mathrm{C}^{\infty}(\bar{Q})$

$$
\begin{cases}v_{t}-\Delta v+a_{0} v=f, & \text { em } Q\left(f \in \mathrm{C}^{\infty}(\bar{Q})\right) \\ v(x, 0)=g(x), & \text { em } \Omega(g \in \mathcal{D}(\Omega)) \\ v(x, t)=h(x, t), & \text { em } \partial \Omega \times[0, T]\left(h \in \mathrm{C}^{\infty}(\partial \Omega \times[0, T])\right)\end{cases}
$$

onde $T>0$ é finito e onde $a_{0}(x, t) \geq 0$ em $Q$. Então para todo $k \in \mathbb{N}$ existe um polinômio $P_{k} \in \mathcal{P}[X]$ com coeficientes independente de $a_{0}, f, g$ e $h$ tal que

$$
\|v\|_{\mathrm{C}^{k}(\bar{Q})} \leq\left(\|f\|_{\mathrm{C}^{2 k+1}(\bar{Q})}+\|g\|_{\mathrm{C}^{2 k}(\bar{\Omega})}+\|h\|_{\mathrm{C}^{2 k+1}(\partial \Omega \times[0, T])}\right) P_{k}\left(\left\|a_{0}\right\|_{\mathrm{C}^{2 k+1}(\bar{Q})}\right) .
$$

O teorema que segue nos dá a unicidade de solução, ou seja, é com este teorema que podemos decidir que o limite da seqüência $\left\{\hat{u}_{n}\right\}_{n \in \mathbb{N}} \subset \mathcal{G}_{f}(\bar{Q})$ solução de (4.9) é única. 
Teorema 4.2.4 (Ver [10]). A solução de (4.9) é única.

Observação 4.2.5. Note que as funções polinomiais que aparecem nos Lemas 4.2.1 $e$ 4.2.3 não dependem do parâmetro $\varphi$ das funções generalizadas em questão.

Como podemos ver Colombeau e Langlais, em seu artigo [10], usaram técnicas clássicas para mostrar que com a teoria das funções generalizadas em $[7,13,11,9]$ podemos obter solução e unicidade do P.V.I.F. (4.9) desde que o dado inicial seja uma função generalizada de suporte compacto. Na próxima seção vamos usar a topologia desenvolvida em nosso trabalho, que são os fatos de densidade e completude (ver Capítulo 3), para mostrar que o mesmo permanece válido sem que o dado inicial tenha, necessariamente, suporte compacto.

\subsection{Uma aplicação das topologias cortantes $\left(\mathcal{G}_{f}(\Omega), \tau_{\Omega f}\right)$ e $\left(\mathcal{G}_{f}(\bar{Q}), \tau_{\bar{Q} b}\right)$}

Nesta seção vamos fazer o prometido no início deste capítulo, isto é, generalizar os resultados contidos nos Teoremas 4.2.2 e 4.2.4. Para isto vamos usar dois resultados topológicos obtidos no Capítulo 3, a saber: $\mathcal{G}_{f_{c}}(\Omega)$ é denso em $\mathcal{G}_{f}(\Omega)$ (Corolário 3.3.9) e $\mathcal{G}_{f}(\bar{\Omega})$ é completo (Teorema 3.4.13). No que segue, para cada $\varphi \in \mathcal{A}_{0}(\mathbb{K})$ fixa, temos

(i) $\hat{u}_{0 n}=\hat{u}_{0 n}(\varphi, \cdot) \in \mathcal{D}(\Omega)$;

(ii) $\hat{u}_{n}=\hat{u}_{n}(\varphi, \cdot, *) \in \mathrm{C}^{\infty}(\bar{Q})$, onde $\bar{Q}=\bar{\Omega} \times[0, T]$;

(iii) $\hat{u}_{n m}=\hat{u}_{n m}(\varphi, \cdot, *)=\hat{u}_{n}(\varphi, \cdot, *)-\hat{u}_{m}(\varphi, \cdot, *) \in \mathrm{C}^{\infty}(\bar{Q})$;

(iv) $\hat{a}_{0}=\hat{a}_{0}(\varphi, \cdot, *)=\hat{u}_{n}^{2}(\varphi, \cdot, *)+\hat{u}_{m}^{2}(\varphi, \cdot, *)+\hat{u}_{n}(\varphi, \cdot, *) \hat{u}_{m}(\varphi, \cdot, *) \geq 0 \mathrm{em}$ $Q=\Omega \times] 0, T[$.

O objetivo das relações acima é simplificar a notação. É na forma que aparecem em (i), (ii), (iii) e (iv) que podemos aplicar os resultados de Colombeau e Langlais [10], vistos na Seção 4.2; principalmente os Lemas 4.2.1 e 4.2.3. Observe que elas vão aparecer, naturalmente, na prova do teorema que segue: 
Teorema 4.3.1. Suponhamos que o dado inicial do PVIF (4.9) $u_{0} \in \mathcal{G}_{f}(\Omega)$. Então existe uma única $u \in \mathcal{G}_{f}(\bar{Q})$ tal que u é solução de (4.9) com dado inicial $u_{0}$.

Prova. Seja $u_{0} \in \mathcal{G}_{f}(\Omega)$. Então existe uma seqüência $\left\{u_{0 n}\right\}_{n \in \mathbb{N}} \subset \mathcal{G}_{f_{c}}(\Omega)$ tal que

$$
u_{0 n} \underset{n \rightarrow \infty}{\longrightarrow} u_{0}
$$

pois pelo Corolário 3.3.9, temos que $\mathcal{G}_{f_{c}}(\Omega)$ é denso em $\mathcal{G}_{f}(\Omega)$. Agora, pelo Teorema 4.2.2, para cada $n \in \mathbb{N}$ existe $u_{n} \in \mathcal{G}_{f}(\bar{Q})$ tal que $u_{n}$ é solução de (4.9) com dado inicial $u_{0 n}$ correspondente. Mas isto é conseqüência do fato que $\hat{u}_{0 n}$ representante de $u_{0 n}$ está em $\mathcal{D}(\Omega)$ e $\hat{u}_{n}$ representante de $u_{n}$ está em $\mathrm{C}^{\infty}(\bar{Q})$ e é uma solução clássica de (4.7) com dado inicial $\hat{u}_{0 n}$. E, portanto, pelo Lema 4.2 .1 para todo $k \in \mathbb{N}$ existe um polinômio $P_{k} \in \mathcal{P}[X]$ tal que $\hat{u}_{n}$ satisfaz (4.8), i.e.,

$$
\left\|\hat{u}_{n}\right\|_{\mathrm{C}^{k}(\bar{Q})} \leq P_{k}\left(\left\|\hat{u}_{0 n}\right\|_{\mathrm{C}^{2 k+1}(\bar{\Omega})}\right)
$$

Observe que agora, temos uma seqüência de soluções $\left\{u_{n}\right\}_{n \in \mathbb{N}} \subset \mathcal{G}_{f}(\bar{Q})$ do PVIF (4.9) associado à seqüência de dados iniciais $\left\{u_{0 n}\right\}_{n \in \mathbb{N}} \subset \mathcal{G}_{f_{c}}(\Omega)$. Vamos mostrar que existe $u \in \mathcal{G}_{f}(\bar{Q})$ tal que $u_{n} \underset{n \rightarrow \infty}{\longrightarrow} u$, i.e., $\left\{u_{n}\right\}_{n \in \mathbb{N}}$ é uma seqüência convergente em $\mathcal{G}_{f}(\bar{Q})$. Como $\mathcal{G}_{f}(\bar{Q})$ é completo (ver Capítulo 3 Teorema 3.4.13) basta mostrarmos que $\left\{u_{n}\right\}_{n \in \mathbb{N}}$ é uma seqüência de Cauchy. Seja $u_{n m}=u_{n}-u_{m}$. Então $u_{n m}$ é uma solução generalizada do seguinte PVIF:

$$
\begin{cases}\left(u_{n m}\right)_{t}-\Delta u_{n m}+a_{0} u_{n m}=0, & \text { em } \mathcal{G}_{f}(\bar{Q}) \\ \left.u_{n m}\right|_{\Omega \times\{0\}}=u_{0 n}-u_{0 m}, & \text { em } \mathcal{G}_{f}(\Omega) \\ \left.u_{n m}\right|_{\partial \Omega \times[0, T]}=0, & \text { em } \mathcal{G}_{f}(\partial \Omega \times[0, T]),\end{cases}
$$

onde $a_{0}=u_{n}^{2}+u_{m}^{2}+u_{n} u_{m}=\left(u_{n}+\frac{1}{2} u_{m}\right)^{2}+\frac{3}{4} u_{m}^{2}$. Como $\hat{u}_{n m}$, representante de $u_{n m}$, é uma função $\mathrm{C}^{\infty}(\bar{Q})$ solução clássica do PVIF:

$$
\begin{cases}\left(\hat{u}_{n m}\right)_{t}-\Delta \hat{u}_{n m}+\hat{a}_{0} \hat{u}_{n m}=0, & \text { em } Q \\ \left.\hat{u}_{n m}\right|_{\Omega \times\{0\}}=\hat{u}_{0 n}-\hat{u}_{0 m}, & \text { em } \Omega \\ \left.\hat{u}_{n m}\right|_{\partial \Omega \times[0, T]}=0, & \text { em } \partial \Omega \times[0, T],\end{cases}
$$

onde $T>0$ é finito e $\hat{a}_{0}=\hat{u}_{n}^{2}+\hat{u}_{m}^{2}+\hat{u}_{n} \hat{u}_{m}=\left(\hat{u}_{n}+\frac{1}{2} \hat{u}_{m}\right)^{2}+\frac{3}{4} \hat{u}_{m}^{2} \geq 0$ em $Q$, segue pelo Lema 4.2 .3 que para todo $k \in \mathbb{N}$ existe um polinômio $P_{k} \in \mathcal{P}[X]$ com coeficientes 
independentes de $\hat{a}_{0}$ e $\hat{u}_{0 n}-\hat{u}_{0 m}$ tal que

$$
\left\|\hat{u}_{n m}\right\|_{\mathrm{C}^{k}(\bar{Q})} \leq\left\|\hat{u}_{0 n}-\hat{u}_{0 m}\right\|_{\mathrm{C}^{2 k}(\bar{\Omega})} P_{k}\left(\left\|\hat{a}_{0}\right\|_{\mathrm{C}^{2 k+1}(\bar{Q})}\right) .
$$

Como $u_{n m}=u_{n}-u_{m}$ segue que $\hat{u}_{n m}=\hat{u}_{n}-\hat{u}_{m}$ e, portanto, podemos escrever (4.15) como segue:

$$
\left\|\hat{u}_{n}-\hat{u}_{m}\right\|_{\mathrm{C}^{k}(\bar{Q})} \leq\left\|\hat{u}_{0 n}-\hat{u}_{0 m}\right\|_{\mathrm{C}^{2 k}(\bar{\Omega})} P_{k}\left(\left\|\hat{a}_{0}\right\|_{\mathrm{C}^{2 k+1}(\bar{Q})}\right)
$$

Tem-se que

$$
\begin{aligned}
\left\|u_{0 n}-u_{0 m}\right\|_{\beta} & \leq\left\|\hat{u}_{0 n}-\hat{u}_{0 m}\right\|_{\mathrm{C}^{2 k}(\bar{\Omega})} \\
& =\sum_{|\sigma| \leq k}\left\|\partial^{\sigma}\left(\hat{u}_{0 n}-\hat{u}_{0 m}\right)(\varphi, \cdot)\right\|_{\bar{\Omega}} \\
& =\sum_{|\sigma| \leq k}\left\|u_{0 n}-u_{0 m}\right\|_{\sigma}
\end{aligned}
$$

este último fator em (4.17) tende a zero quando $n, m \rightarrow \infty$ e, portanto, resta mostrar que

$$
P_{k}\left(\left\|\hat{a}_{0}\right\|_{\mathrm{C}^{2 k+1}(\bar{Q})}\right)
$$

em (4.16) é limitado para mostrarmos que $\left\{u_{n}\right\}_{n \in \mathbb{N}} \subset \mathcal{G}_{f}(\bar{Q})$ é uma seqüência de Cauchy em $\mathcal{G}_{f}(\bar{Q})$. Para isto vamos mostrar que (4.18) é limitado trabalhando com a desigualdade em (4.12), pois $\hat{a}_{0}=\hat{u}_{n}^{2}+\hat{u}_{m}^{2}+\hat{u}_{n} \hat{u}_{m}=\left(\hat{u}_{n}+\frac{1}{2} \hat{u}_{m}\right)^{2}+\frac{3}{4} \hat{u}_{m}^{2}$ e a topologia sobre $\mathcal{G}_{f}(\bar{Q})$ é compatível com a estrutura de álgebra. (Ver [4].) Vamos mostrar que $\left\{u_{n}\right\}_{n \in \mathbb{N}} \subset \mathcal{G}_{f}(\bar{Q})$ é uma seqüência limitada. Sabemos por (4.12) que para todo $n \in \mathbb{N}$ e para todo $\beta \in \mathbb{N}^{n}$, temos que

$$
\begin{aligned}
\left\|u_{n}\right\|_{\beta} & \leq\left\|\hat{u}_{n}\right\|_{\mathrm{C}^{k}(\bar{Q})} \\
& \leq P_{k}\left(\left\|\hat{u}_{0 n}\right\|_{\mathrm{C}^{2 k+1}(\bar{\Omega})}\right) \\
& =P_{k}\left(\sum_{|\sigma| \leq 2 k+1}\left\|\partial^{\sigma} \hat{u}_{0 n}(\varphi, \cdot)\right\|_{\bar{\Omega}}\right) \\
& =P_{k}\left(\sum_{|\sigma| \leq 2 k+1}\left\|u_{0 n}\right\|_{\sigma}\right)
\end{aligned}
$$

onde o polinômio $P_{k}$ é independente de $\varphi \in \mathcal{A}_{0}(\mathbb{K})$. Como $u_{0 n} \underset{n \rightarrow \infty}{\longrightarrow} u_{0}$ segue que

$$
\sum_{|\sigma| \leq 2 k+1}\left\|u_{0 n}\right\|_{\sigma} \underset{n \rightarrow \infty}{\longrightarrow} \sum_{|\sigma| \leq 2 k+1}\left\|u_{0}\right\|_{\sigma}
$$


e, portanto, existe $C=C(\varphi)>0$ tal que

$$
\sum_{|\sigma| \leq 2 k+1}\left\|u_{0 n}\right\|_{\sigma} \leq C, \forall n \in \mathbb{N} .
$$

Isto mostra que o argumento do polinômio $P_{k}$ em (4.19) é limitado. Agora, notemos que o coeficiente do termo de maior grau de $P_{k}$ é positivo, pois se não para valores arbitrariamente grandes de (4.21) teríamos

$$
P_{k}\left(\sum_{|\sigma| \leq 2 k+1}\left\|u_{0 n}\right\|_{\sigma}\right)<0
$$

o que é um absurdo, pois isto contradiz a desigualdade em (4.19), já que $\left\|u_{n}\right\|_{\beta}>0$. Daí, existem $C^{\prime}>C$ e $x_{0}>0$ tais que $C^{\prime} \in \mathbb{R}$ e para todo $x \in \mathbb{R}$ com $x_{0}<x<C^{\prime}$ tem-se que $P_{k}(x)<P_{k}\left(C^{\prime}\right)$ e; portanto, temos por (4.21) que

$$
\sum_{|\sigma| \leq 2 k+1}\left\|u_{0 n}\right\|_{\sigma} \leq C^{\prime} \Rightarrow P_{k}\left(\sum_{|\sigma| \leq 2 k+1}\left\|u_{0 n}\right\|_{\sigma}\right)<P_{k}\left(C^{\prime}\right) .
$$

Agora, por (4.19) e (4.22), temos que

$$
\left\|u_{n}\right\|_{\beta} \leq P_{k}\left(C^{\prime}\right)=M, \forall n \in \mathbb{N} .
$$

e, portanto, $\left\{u_{n}\right\}_{n \in \mathbb{N}} \subset \mathcal{G}_{f}(\bar{Q})$ é uma seqüência limitada. Vamos agora voltar para o problema de limitar a expressão em (4.18). Sendo $u_{n}$ limitada para todo $n \in \mathbb{N}$ segue desenvolvendo (4.18) que

$$
\begin{aligned}
P_{k}\left(\left\|\hat{a}_{0}\right\|_{\mathrm{C}^{2 k+1}(\bar{Q})} \|\right) & =P_{k}\left(\sum_{|\sigma| \leq 2 k+1}\left\|\partial^{\sigma} \hat{a}_{0}(\varphi, \cdot)\right\|_{\bar{Q}}\right) \\
& =P_{k}\left(\sum_{|\sigma| \leq 2 k+1}\left\|a_{0}\right\|_{\sigma}\right) \\
& =P_{k}\left(\sum_{|\sigma| \leq 2 k+1}\left\|u_{n}^{2}+u_{m}^{2}+u_{n} u_{m}\right\|_{\sigma}\right)
\end{aligned}
$$

lembrando que $a_{0}=u_{n}^{2}+u_{m}^{2}+u_{n} u_{m}$. Como cada uma das parcelas em $\left\|u_{n}^{2}+u_{m}^{2}+u_{n} u_{m}\right\|_{\sigma}$ é limitada, pois $\left\{u_{n}\right\}_{n \in \mathbb{N}}$ é limitada segue que

$$
\sum_{|\sigma| \leq 2 k+1}\left\|u_{n}^{2}+u_{m}^{2}+u_{n} u_{m}\right\|_{\sigma}
$$


é limitado. E, portanto, usando o mesmo argumento que antes para $P_{k}$, obtemos pelo fato que a expressão (4.24) é limitada que

$$
P_{k}\left(\sum_{|\sigma| \leq 2 k+1}\left\|u_{n}^{2}+u_{m}^{2}+u_{n} u_{m}\right\|_{\sigma}\right)
$$

é limitado e, portanto, por (4.23), temos que a expressão em (4.18) é limitada. E isto é exatamente o que queríamos para concluir que $\left\{u_{n}\right\}_{n \in \mathbb{N}} \subset \mathcal{G}_{f}(\bar{Q})$ é uma seqüência de Cauchy em $\mathcal{G}_{f}(\bar{Q})$. Finalmente, como $\mathcal{G}_{f}(\bar{Q})$ é completo (ver Capítulo 3, Teorema 3.4 .13 com prova em $([4]))$ segue que existe $u \in \mathcal{G}_{f}(\bar{Q})$ tal que $u_{n} \underset{n \rightarrow \infty}{\longrightarrow} u$. Resta agora, mostrar que a função generalizada $u \in \mathcal{G}_{f}(\bar{Q})$, limite das $u_{n} \in \mathcal{G}_{f}(\bar{Q})$ é a solução do PVIF (4.9) com dado inicial $u_{0} \in \mathcal{G}_{f}(\Omega)$. Para isto, seja $P(u)=u_{t}-\Delta u+u^{3}$, então precisamos mostrar que $0=P\left(u_{n}\right) \underset{n \rightarrow \infty}{\longrightarrow} P(u)$. Mas isto é imediato da continuidade do operador derivação (ver 3.3.2 no Capítulo 3) e da continuidade da multiplicação em $\mathcal{G}_{f}(\bar{Q})$. 


\section{Referências Bibliográficas}

[1] J. Aragona, Colombeau generalized functions on quasi-regular sets, Publ. Math. Debrecen, 68 (2006), pp. 371-399.

[2] J. Aragona And H. A. Biagioni, Intrinsic definition of the Colombeau algebra of generalized functions, Anal. Math., 17 (1991), pp. 75-132.

[3] J. Aragona, R. Fernandez, and S. O. JuriaAns, The sharp topology on the full Colombeau algebra of generalized functions, Integral Transforms Spec. Funct., 17 (2006), pp. 165-170.

[4] J. Aragona, A. R. G. Garcia, And O. S. JuriaAns, A completude das álgebras de colombeau $\mathcal{G}_{f}(\omega)$ e $\mathcal{G}_{f}(\bar{O})$, onde $\omega$ e $O$ são abertos de $\mathbb{R}^{n}$ e $O$ é limitado. Manuscrito, IME-USP, São Paulo S.P., Brazil, 2006.

[5] J. Aragona And S. O. JuRIAANs, Some structural properties of the topological ring of Colombeau's generalized numbers, Comm. Algebra, 29 (2001), pp. 2201-2230.

[6] J. Aragona, S. O. Jurianans, O. R. B. Oliveira, and D. Scarpalézos, Algebraic and geometric theory of the topological ring of Colombeau's generalized functions. Submitted.

[7] H. A. Biagioni, Introduction to a nonlinear theory of generalized functions. Notas de matemática, Unicamp, Campinas S.P., Brazil, 1988.

[8] H. BRÉzIS AND A. FRIEDMAN, Nonlinear parabolic equations involving measures as initial conditions, J. Math. Pures Appl. (9), 62 (1983), pp. 73-97. 
[9] J.-F. Colombeau, Elementary introduction to new generalized functions, vol. 113 of North-Holland Mathematics Studies, North-Holland Publishing Co., Amsterdam, 1985. Notes on Pure Mathematics, 103.

[10] J.-F. Colombeau AND M. LANGlais, Generalized solutions of nonlinear parabolic equations with distributions as initial conditions, J. Math. Anal. Appl., 145 (1990), pp. 186-196.

[11] J.-F. Colombeau And A. Y. LeRoux, Multiplications of distributions in elasticity and hydrodynamics, J. Math. Phys., 29 (1988), pp. 315-319.

[12] M. Kunziger, Lie transformation groups in Colombeau algebras, PhD thesis, Fakultät der Universität Wien, 1996.

[13] E. E. Rosinger, Generalized solutions of nonlinear partial differential equations, vol. 146 of North-Holland Mathematics Studies, North-Holland Publishing Co., Amsterdam, 1987. Notas de Matemática [Mathematical Notes], 119.

[14] D. ScarpalÉzos, Topologies dans les espaces de nouvelles fonctions generalisées de colombeau. $\overline{\mathbb{C}}$-modules topologiques. Université Paris 7, 1993.

[15] —-, Colombeau's generalized functions: topological structures; microlocal properties. A simplified point of view. I, Bull. Cl. Sci. Math. Nat. Sci. Math., (2000), pp. 89-114.

[16] D. ScarpalÉzos, Colombeau's generalized functions: topological structures; microlocal properties. A simplified point of view. II, Publ. Inst. Math. (Beograd) (N.S.), 76(90) (2004), pp. 111-125.

[17] A. A. D. Silva, Sobre a álgebra simplificada das funções generalizadas de colombeau, master's thesis, Universidade de São Paulo; Instituto de Matemática e Estatística, 2005 . 


\section{Índice Remissivo}

álgebra das funções generalizadas plenas de Colombeau, 57, 59

álgebra das funções generalizadas simplificadas de Colombeau, 57

álgebra das funções holomorfas generalizadas plenas de Colombeau, 75

aberto, 9,43

anel, 54

anel com unidade, 41

anel comutativo, $52,53,55$

anel dos números generalizados pleno de

Colombeau, 9 anel quociente, 20, 59

anel topológico completo, 20, 23

anti-simétrica, 52

aplicação natural, 27, 49

aplicações contínuas, 24

argumento, 49

associação, 9,10

base enumerável, 30

cadeia, 48

característica zero, 45

característica zero, 45

classe de equivalência, 14

anel dos números generalizados plenos Colombeađolombeau, 49

28

anel dos números generalizados plenos de

Colombeau, 47

anel dos números generalizados plenos de

Colombeau, 12, 21, 38

anel dos números generalizados simplifi-

cados de Colombeau, 21, 47

anel dos números generalizados simplifi-

cados de Colombeua, 13

anel local, 45 compacto, 26, 27

completa, 57

completude, 57

conjugação, 51

conjunto parcialmente ordenado, 48

conjunto das unidades, 9

conjunto de zeros, 28, 33

conjunto dos idempotentes, 53

conjunto limitado, 26

conjunto parcialmente ordenado, 48 
conjunto raro, 9, 25

conjunto totalmente ordenado, 48

conjuntos de zero, 34

corpo, 12

corpo de classe residual, 47

densidade, 57

denso, 9,43

desigualdade triangular, 53

desigualdade triangular, 50

diâmetro do suporte, 12

divisor de zero, $34,36,38$

domínio regular limitado, 89

domínios integrais, 9, 27

elementos comparáveis, 48

elementos invertíveis, 34

elementos nilpotentes, 27

epimorfismos, 51

equação parabólica, 89

equação parabólica não linear, 91

espaço vetorial de dimensão finita, 10

espaços normados, suaves de dimensão finita,

\section{0}

estável, 30

estrutura uniforme, 17

estrutura uniforme cortante, 71

existência, 57

extensão própria, 27

fechado, 9,25

função generalizada com suporte compacto,

89

funções características, 28

funções características, 9,28 funções generalizadas plenas de Colombeau, 92

grupo das unidades, 28

Hausdorff, 24, 26, 57

idéias primos, 27

ideais maximais, $9,28,53$

ideais próprio aberto, 26

ideais primos, $9,28,53$

ideais principais, 54

ideal, 50

ideal maximal, 41

ideal aberto, 26

ideal aberto próprio, 26

ideal idempotente, 55

ideal maximal, 9, 25, 27, 41-43, 45, 56

ideal próprio, 25, 26, 31, 33, 43

ideal primo, 29, 32, 33, 42, 55, 56

idempotente, 31,54

idempotente não-trivial, 54

idempotentes ortogonais, 33

idempotentes ortogonais, 29

igualdade no sentido fraco, 10

imersão constante, 12

incomparáveis, 48

invariante por conjugação, 51

invariante por translação, 14, 16

inversa, 49

invertíveis, 42

involução, 51

kernel, 27

lei de fronteira, 91 
módulo topológico, 26

moderada, 35

moderadas, 11, 40, 49

multiplicação, 24

número associado, 12

número generalizado pleno, 49

não-negativo, 52

não-unidade, 37

nil-radical, 9, 27, 52, 55, 56

norma, 10

nulas, 12,36

parabólico, 57

parcialmente ordenado, 52

parte real, 51

polinômio, 92

polinômios $k$-homogêneos, 11

problema de Cauchy, 91

problema de valor inicial e de fronteiraPVIF, 89

problema de valor inicial e de fronteira, 57

PVIF, 57

q-negativa, 49

q-negativo, 49, 52

q-positiva, 49

q-positivo, 49,52

quase-negativo, 48

quase-positivo, 48

quase-regular, 64

radical de Jacobson, 41

reflexiva, 52 regra de Leibnitz, 67

relação binária, 48

relação de ordem total, 52

relação de equivalência, 10

relação de ordem, 52

relação de ordem parcial, 47,48

relação de ordem total, 48

seqüência de Cauchy, 23, 24

solução generalizada, 89

soma, 24

sombra, 12

subanel, 11

subconjunto aberto, 24

subconjunto limitado, 26

subcorpo, 27

submódulos próprio aberto, 26

Teorema da aproximação, 9, 38, 43

topologia, 9

topologia cortante plenas, 57

topologia escalar constante, 17

totalmente ordenado, 52

transitiva, 53

ultra-métrica, 14, 16

união finita, 30, 31

unicidade, 57

unidade, $37,52-55$

valor absoluto, 49

valuação, 13

Von Neumann regular, 53-56 